\title{
Gravito-electromagnetic analogies
}

\author{
L. Filipe O. Costa ${ }^{1,2 *}$ José Natário ${ }^{2 \dagger}$ \\ ${ }^{1}$ Centro de Física do Porto, Universidade do Porto \\ Rua do Campo Alegre, 687, 4169-007 Porto, Portugal \\ ${ }^{2}$ Centro de Análise Matemática, Geometria e Sistemas Dinâmicos, \\ Instituto Superior Técnico, 1049-001, Lisboa, Portugal
}

October 28, 2014

\begin{abstract}
We reexamine and further develop different gravito-electromagnetic (GEM) analogies found in the literature, and clarify the connection between them. Special emphasis is placed in two exact physical analogies: the analogy based on inertial fields from the so-called " $1+3$ formalism", and the analogy based on tidal tensors. Both are reformulated, extended and generalized. We write in both formalisms the Maxwell and the full exact Einstein field equations with sources, plus the algebraic Bianchi identities, which are cast as the source-free equations for the gravitational field. New results within each approach are unveiled. The well known analogy between linearized gravity and electromagnetism in Lorentz frames is obtained as a limiting case of the exact ones. The formal analogies between the Maxwell and Weyl tensors are also discussed, and, together with insight from the other approaches, used to physically interpret gravitational radiation. The precise conditions under which a similarity between gravity and electromagnetism occurs are discussed, and we conclude by summarizing the main outcome of each approach.
\end{abstract}

Keywords: Gravitomagnetism - Bel decomposition - Tidal tensors · Inertial forces · 1+3 Splitting · Quasi-Maxwell formalism · Gyroscope precession · Spin-curvature force

\section{Contents}

1 Introduction

1.1 Notation and conventions ........................ 4

2 The gravito-electromagnetic analogy based on tidal tensors 5

2.1 Tidal tensor formulation of Maxwell and Einstein equations . . . . . . . . . . 6 6

2.1 .1 Maxwell's equations . . . . . . . . . . . . . . . 7

2.1 .2 Einstein's equations . . . . . . . . . . . . . . . 8

2.2 Gravity vs Electromagnetism . . . . . . . . . . . . . . . . 11

2.3 The analogy for differential precession . . . . . . . . . . . . . 14

*Ifpocosta@math.ist.utl.pt

†jnatar@math.ist.utl.pt 
3 Gravito-electromagnetic analogy based on inertial fields from the $1+3$ splitting of spacetime

3.1 The reference frame . . . . . . . . . . . . . . . . . 18

3.2 Geodesics. Inertial forces — "gravitoelectromagnetic fields" . . . . . . . . . . . . . 21

3.2.1 The derivative operator $\tilde{D} / d \tau$ and inertial forces . . . . . . . . . . . . . . 22

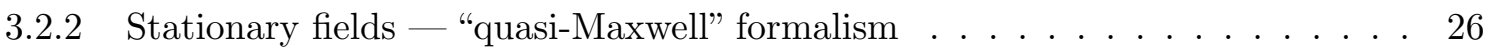

3.3 Gyroscope precession . . . . . . . . . . . . . . . . . . . 26

3.4 Field equations . . . . . . . . . . . . . . . . . . . . 28

3.4.1 Maxwell equations for the electromagnetic fields measured by an arbitrary congruence of observers . . . . . . . . . . . . . . . 29 29

3.4 .2 Einstein equations . . . . . . . . . . . . . . . . . . 30

3.4.3 Special cases: quasi-Maxwell regime $(1+3$ formalism), and hypersurface orthogonal observers $(3+1$ formalism $) \ldots \ldots \ldots . \ldots . \ldots . \ldots 32$

3.5 Relation with tidal tensor formalism . . . . . . . . . . . . . . . . 34

3.6 Force on a gyroscope . . . . . . . . . . . . . . . . . . 37

4 "Ultra-stationary" spacetimes

4.1 The Gödel Universe . . . . . . . . . . . . . . . . . . . . . . . 40

5 Linear gravitoelectromagnetism 41

6 The formal analogy between gravitational tidal tensors and electromagnetic fields

6.1 Matte's equations vs Maxwell equations. Tidal tensor interpretation of gravitational radiation. . . . . . . . . . . . . . . . . . . . 47

6.1 .1 Super-energy . . . . . . . . . . . . . . . . . . . 49

6.2 The relationship with the other GEM analogies . . . . . . . . . . . . 49

7 When can gravity be similar to electromagnetism?

8 Conclusion

A Inertial Forces — simple examples in flat spacetime 53

\section{Introduction}

This work has two main goals: one is to establish the connection between the several gravitoelectromagnetic analogies existing in the literature, summarizing the main results and insights offered by each of them; the second is to further develop and extend some of these analogies.

In an earlier work by one of the authors [1, 2, a gravito-electromagnetic analogy based on tidal tensors was presented, and its relationship with 1) the well known analogy between linearized gravity and electromagnetism, 2) the mapping, via the Klein-Gordon equation, between ultrastationary spacetimes and magnetic fields in curved spacetimes, and 3) the formal analogies between the Weyl and Maxwell tensors (their decomposition into electric and magnetic parts, the quadratic scalar invariants they form, and the field equations they obey) was discussed. 
Building up on the work in [2], another approach is herein added to the discussion: the exact analogy based on the fields of inertial forces, arising in the context of the $1+3$ splitting of spacetime. This approach, which is herein reformulated and suitably generalized, is still not very well known, but very far reaching. It is therefore important to understand how it relates with the other known analogies, and in particular with the (also exact) approach based on tidal tensors.

Each of the analogies discussed here are also further developed, and some new results within each of them are presented. We start in Sec. 2 by revisiting the approach based on tidal tensors introduced in [1] (and partly reviewed in [3]), completing it by extending the formalism to the full gravitational field equations (cast herein as the Einstein field equations, plus the algebraic Bianchi identities). More precisely, through suitable projector techniques, we make a full $1+3$ covariant splitting of the latter, obtaining a set of six algebraic equations: four of them involve only the sources, the "gravitoelectric" $\left(\mathbb{E}_{\alpha \beta}\right)$ and "gravitomagnetic" $\left(\mathbb{H}_{\alpha \beta}\right)$ tidal tensors, and are formally similar to the Maxwell equations written in this formalism; plus an additional pair of equations that involve the purely spatial curvature (encoded in the tensor $\mathbb{F}_{\alpha \beta}$ ) and have no electromagnetic analogue. The formalism is then used to contrast the gravitational and electromagnetic tidal effects. We also add to the list of exact analogies manifest in this formalism the one concerning relative precession of spinning particles.

In Sec. 3, we discuss another exact gravito-electromagnetic analogy, the one drawing a parallelism between spatial inertial forces - described by the "gravitoelectromagnetic" (GEM) fields and the electromagnetic fields. GEM fields are best known from linearized theory, e.g. [7, 8, 9, 10, 11, 12, 13, 14, 15, 16, 17. Less well known are the exact analogies based on inertial fields that arise in the splitting of spacetime into time + space with respect to two preferred congruences of observers: time-like Killing congruences in stationary spacetimes, introduced by Landau-Lifshitz [18, and further worked out by other authors [19, 20, 21, 22, 23, 24], which leads to the so-called "quasi-Maxwell" analogy; and hypersurface orthogonal observers, which have motivated substantially different approaches, e.g. [25, 26], leading also to analogies based on exact equations, albeit not as close as in the former case. Lesser known still is the existence of an exact formulation applying to arbitrary observer congruences in arbitrary spacetimes [27, 28], where a framework is developed to encompass the inertial fields arising in the different spacetime splittings in the literature. Herein we propose a reformulation of the problem, also applying to arbitrary observers in arbitrary spacetimes, but whose most distinctive feature is allowing for an arbitrary rotation of the spatial frame, which was achieved by defining a suitable connection $(\tilde{\nabla})$ on the bundle of vectors orthogonal to a congruence of time-like curves. That allows to describe the inertial forces of any orthonormal frame, and manifests that the so-called "gravitomagnetic field" $(\vec{H})$ consists of a combination of two effects of different (independent, in a general formulation) origin: the vorticity $\vec{\omega}$ of the observer congruence, plus the angular velocity $\vec{\Omega}$ of rotation along the congruence (relative to Fermi-Walker transport) of the triad of spatial axes that each observer "carries". Such formulation encompasses the gravitomagnetic field of different frames studied in the literature, e.g. the one measured by the Killing (or "static") observers in a stationary spacetime, studied in e.g. [20, 23, 24, or the one arising in the so-called "locally non-rotating frames" (LNR) in e.g. [26, 103]. Drawing a parallelism with what is done in Sec. 2, we express the Maxwell equations and the full gravitational field equations in this formalism. Again, a set of four equations are produced which exhibit many similarities with their electromagnetic counterparts (closer in the case of rigid observer congruences 
in stationary spacetimes, with an almost one to one correspondence), plus two additional equations which have no electromagnetic analogue. We also add to the list of exact analogies the one between the electromagnetic force on a magnetic dipole and the gravitational force on a gyroscope, when both are at rest with respect to a rigid (arbitrarily accelerated and rotating) frame in a stationary spacetime. In Sec. 3.5 we establish the relationship between the inertial fields and the tidal tensors of Sec. 2 - a particularly important result in the context of this work.

In Sec. 4 we discuss a special class of spacetimes admitting global rigid geodesic congruences, the "ultra-stationary" spacetimes. They have interesting properties in the context of GEM, which were discussed in [1] in the framework of the tidal tensor analogy; herein we revisit those spacetimes in the framework of the GEM inertial fields of Sec. 3, shedding some new light on questions left open in [1].

In Sec. 5 we explain the relation between the exact approach based in the inertial GEM fields of Sec. 3, and the popular gravito-electromagnetic analogy based on linearized theory, e.g. [7, 8, 10, 9]. The latter is obtained as a special limit of the exact equations of the former. Taking this route gives a clearer account of the physical meaning of the GEM fields, which are often somewhat naively derived from the temporal components of the metric tensor (drawing a parallelism with the electromagnetic potentials), without making apparent their status as artifacts of the reference frame, and in particular their relation with the kinematical quantities associated to the observer's congruence. It is also a procedure for obtaining the field equations that does not rely on choosing the harmonic gauge condition and its inherent subtleties (which have been posing some difficulties in the literature, see e.g. [9, 30, 10, 2]).

In Sec. 6 we briefly review the formal analogies between the electric $\left(\mathcal{E}_{\alpha \beta}\right)$ and magnetic $\left(\mathcal{H}_{\alpha \beta}\right)$ parts of the Weyl tensor, and the electric $\left(E^{\alpha}\right)$ and magnetic $\left(B^{\alpha}\right)$ fields, e.g. [31, 32, 33, 34, 35, 36, 37, 38, 39, 40, 41, 42, 75. One of its interesting results is that in the case of vacuum, in the linear regime, one obtains a set of equations formally similar to Maxwell's equations in a Lorentz frame, only with the gravitational tidal tensors $\left(\mathcal{E}_{i j}=\mathbb{E}_{i j}, \mathcal{H}_{i j}=\mathbb{H}_{i j}\right)$ in place of $E^{\alpha}$ and $B^{\alpha}$. Formally analogous wave equations follow likewise, whose physical interpretation is discussed based on what is learned from the approaches herein.

\subsection{Notation and conventions}

1. Signature and signs. We use the signature $-+++; \epsilon_{\alpha \beta \gamma \delta} \equiv \sqrt{-g}[\alpha \beta \gamma \delta]$ denotes the Levi-Civita tensor, and we follow the orientation $[1230]=1$ (i.e., in flat spacetime $\left.\epsilon_{1230}=1\right) . \epsilon_{i j k} \equiv \epsilon_{i j k 0}$ is the 3 -D alternating tensor. $\star$ denotes the Hodge dual.

2. Time and space projectors. $\left(\top^{u}\right)_{\beta}^{\alpha} \equiv-u^{\alpha} u_{\beta},\left(h^{u}\right)_{\beta}^{\alpha} \equiv u^{\alpha} u_{\beta}+g_{\beta}^{\alpha}$ are, respectively, the projectors parallel and orthogonal to a unit time-like vector $u^{\alpha}$; may be interpreted as the time and space projectors in the local rest frame of an observer of 4 -velocity $u^{\alpha} .\langle\alpha\rangle$ denotes the index of a spatially projected tensor: $A^{\langle\alpha\rangle \beta \ldots} \equiv\left(h^{u}\right)_{\mu}^{\alpha} A^{\mu \beta \ldots}$.

3. $\rho_{c}=-j^{\alpha} u_{\alpha}$ and $j^{\alpha}$ are, respectively, the charge density and current 4-vector; $\rho=T_{\alpha \beta} u^{\alpha} u^{\beta}$ and $J^{\alpha}=-T_{\beta}^{\alpha} u^{\beta}$ are the mass/energy density and current (quantities measured by the observer of 4-velocity $\left.u^{\alpha}\right) ; T_{\alpha \beta} \equiv$ energy-momentum tensor.

4. $S^{\alpha} \equiv$ spin 4-vector; $\mu^{\alpha} \equiv$ magnetic dipole moment; defined such that their components in 
the particle's proper frame are $S^{\alpha}=(0, \vec{S}), \mu^{\alpha}=(0, \vec{\mu})$. For their precise definition in terms of moments of $T^{\alpha \beta}$ and $j^{\alpha}$, see [6].

5. Tensors as measured by an observer. $\left(A^{u}\right)^{\alpha_{1} . . \alpha_{n}}$ denotes the tensor $\mathbf{A}$ as measured by an observer $\mathcal{O}(u)$ of 4 -velocity $u^{\alpha}$. For example, $\left(E^{u}\right)^{\alpha} \equiv F_{\beta}^{\alpha} u^{\beta},\left(E^{u}\right)_{\alpha \beta} \equiv F_{\alpha \gamma ; \beta} u^{\gamma}$ and $\left(\mathbb{E}^{u}\right)_{\alpha \beta} \equiv$ $R_{\alpha \mu \beta \nu} u^{\nu} u^{\mu}$ denote, respectively, the electric field, electric tidal tensor, and gravitoelectric tidal tensor as measured by $\mathcal{O}(u)$. Analogous forms apply to their magnetic/gravitomagnetic counterparts.

For 3 -vectors we use notation $\vec{A}(u)$; for example, $\vec{E}(u)$ denotes the electric 3 -vector field as measured by $\mathcal{O}(u)$ (i.e., the space part of $\left(E^{u}\right)^{\alpha}$, written in a frame where $\left.u^{i}=0\right)$. Often we drop the superscript (e.g. $\left.\left(E^{U}\right)^{\alpha} \equiv E^{\alpha}\right)$, or the argument of the 3-vector: $\vec{E}(U) \equiv \vec{E}$, when the meaning is clear.

6. Electromagnetic field. The Maxwell tensor $F^{\alpha \beta}$ and its Hodge dual $\star F^{\alpha \beta} \equiv \epsilon_{\mu \nu}^{\alpha \beta} F_{\mu \nu} / 2$ decompose in terms of the electric $\left(E^{u}\right)^{\alpha} \equiv F_{\beta}^{\alpha} u^{\beta}$ and magnetic $\left(B^{u}\right)^{\alpha} \equiv \star F_{\beta}^{\alpha} u^{\beta}$ fields measured by an observer of 4 -velocity $u^{\alpha}$ as

$$
F_{\alpha \beta}=2 u_{[\alpha}\left(E^{u}\right)_{\beta]}+\epsilon_{\alpha \beta \gamma \delta} u^{\delta}\left(B^{u}\right)^{\gamma} \quad \text { (a) } \quad \star F_{\alpha \beta}=2 u_{[\alpha}\left(B^{u}\right)_{\beta]}-\epsilon_{\alpha \beta \gamma \sigma} u^{\sigma}\left(E^{u}\right)^{\gamma}
$$

\section{The gravito-electromagnetic analogy based on tidal tensors}

The rationale behind the tidal tensor gravito-electromagnetic analogy is to make a comparison between the two interactions based on physical forces present in both theories. The electromagnetic Lorentz force has no physical counterpart in gravity, as monopole point test particles in a gravitational field move along geodesics, without any force being exerted on them. In this sense, the analogy drawn in Sec. 3.2 between Eqs. (54) and (52) is a comparison of a physical electromagnetic force to an artifact of the reference frame. Tidal forces, by their turn, are covariantly present in both theories, and their mathematical description in terms of objects called "tidal tensors" is the basis of this approach. Tidal forces manifest themselves in essentially two basic effects: the relative acceleration of two nearby monopole test particles, and in the net force exerted on dipoles. The notions of multipole moments arise from a description of the test bodies in terms of the fields they would produce. In electromagnetism they are the multipole expansions of the 4-current density vector $j^{\alpha}=\left(\rho_{c}, \vec{j}\right)$, rigorously established in [48], and well known in textbooks as the moments of the charge and current densities. In gravity they are are the moments of the energy momentum tensor $T_{\alpha \beta}$, the so called [47] "gravitational skeleton", of which only the moments of the 4-current density $J^{\alpha}=-T^{\alpha \beta} U_{\beta}$ have an electromagnetic counterpart. Monopole particles in the context of electromagnetism are those whose only non-vanishing moment is the total charge; dipole particles are particles with non-vanishing electric and magnetic dipole moments (i.e., respectively the dipole moments of $\rho_{c}$ and $\vec{j}$ ); see [48] and companion paper [6] for precise definitions of these moments. Monopole particles in gravity are particles whose only non-vanishing moment of $T^{\alpha \beta}$ is the mass, and correspond to the usual notion of point test particle, which moves along geodesics. There is no gravitational analogue of the intrinsic electric dipole, as there are no negative masses; but there is an analogue of the magnetic dipole moment, which is the "intrinsic" angular momentum (i.e. the angular momentum about the particle's center of mass), usually dubbed spin vector/tensor. Sometimes we will also call it, for obvious reasons, the "gravitomagnetic dipole moment". A particle 
possessing only pole-dipole gravitational moments corresponds to the notion of an ideal gyroscope. We thus have two physically analogous effects suited to compare gravitational and electromagnetic tidal forces: worldline deviation of nearby monopole test particles, and the force exerted on magnetic dipoles/gyroscopes. An exact gravito-electromagnetic analogy, summarized in Table 1 , emerges from this comparison.

Eqs. (1.1) are the worldline deviations for nearby test particles with the sam $\rrbracket^{1}$ tangent vector (and the same ratio charge/mass in the electromagnetic case), separated by the infinitesimal vector $\delta x^{\alpha}$. They tell us that the so-called (e.g. [45]) electric part of the Riemann tensor $\mathbb{E}_{\beta}^{\alpha} \equiv R_{\mu \beta \nu}^{\alpha} U^{\mu} U^{\nu}$ plays in the geodesic deviation equation $(1,1 \mathrm{~b})$ the same physical role as the tensor $E_{\alpha \beta} \equiv F_{\alpha \gamma ; \beta} U^{\gamma}$ in the electromagnetic worldline deviation (1.1a): in a gravitational field the "relative acceleration" between two nearby test particles, with the same 4-velocity $U^{\alpha}$, is given by a contraction of $\mathbb{E}_{\alpha \beta}$ with the separation vector $\delta x^{\beta}$; just like in an electromagnetic field the "relative acceleration" between two nearby charged particles (with the same $U^{\alpha}$ and ratio $q / m$ ) is given by a contraction of the electric tidal tensor $E_{\alpha \beta}$ with $\delta x^{\alpha}$. $E_{\alpha \beta}$ measures the tidal effects produced by the electric field $E^{\alpha}=F_{\gamma}^{\alpha} U^{\gamma}$ as measured by the test particle of 4 -velocity $U^{\alpha}$. We can define it as a covariant derivative of the electric field as measured in the inertial frame momentarily comoving with the particle: $E_{\alpha \beta}=\left.E_{\alpha ; \beta}\right|_{U=\text { const }}$. Hence we dub it the "electric tidal tensor", and its gravitational counterpart the "gravitoelectric tidal tensor".

Eqs (1,2) are, respectively, the electromagnetic force on a magnetic dipole [6], and the MathissonPapapetrou equation [47, 49] for the gravitational force exerted on a gyroscope (supplemented by the Mathisson-Pirani spin condition [47, 50]; see [6] for more details). They tell us that the magnetic part of the Riemann tensor $\mathbb{H}_{\beta}^{\alpha} \equiv \star R_{\mu \beta \nu}^{\alpha} U^{\mu} U^{\nu}$ plays in the gravitational force $(1.2 \mathrm{~b})$ the same physical role as the tensor $B_{\alpha \beta} \equiv \star F_{\alpha \gamma ; \beta} U^{\gamma}$ in the electromagnetic force (1.2a): the gravitational force exerted on a spinning particle of 4 -velocity $U^{\alpha}$ is exactly given by a contraction of $\mathbb{H}_{\alpha \beta}$ with the spin vector $S^{\alpha}$ (the "gravitomagnetic dipole moment"), just like its electromagnetic counterpart is exactly given by a contraction of the magnetic tidal tensor $B_{\alpha \beta}$ with the magnetic dipole moment $\mu^{\alpha}$. $B_{\alpha \beta}$ measures the tidal effects produced by the magnetic field $B^{\alpha}=\star F_{\gamma}^{\alpha} U^{\gamma}$ as measured by the particle of 4-velocity $U^{\alpha}$; for this reason we dub it the "magnetic tidal tensor", and its gravitational analogue $\mathbb{H}_{\alpha \beta}$ the "gravitomagnetic tidal tensor".

\subsection{Tidal tensor formulation of Maxwell and Einstein equations}

Taking time and space projections, Maxwell's and Einstein's equations can be expressed in tidal tensor formalism; that makes explicit a striking aspect of the analogy: Maxwell's equations (the source equations plus the Bianchi identity) may be cast as a set of algebraic equations involving only tidal tensors and source terms (the charge current 4-vector); and the gravitational field equations (Einstein's source equations plus the algebraic Bianchi identity) as a set of five independent

\footnotetext{
${ }^{1}$ We want to emphasize this point, which, even today, is not clear in the literature. Eqs. 11 1) apply to the instant where the two particles have the same (or infinitesimally close, in the gravitational case) tangent vector. When the particles have arbitrary velocities, both in electromagnetism and gravity, their relative acceleration is not given by a simple contraction of a tidal tensor with a separation vector; the equations include more terms, see [1, 2], 53. There is however a difference: whereas Eq. (1,1a) requires strictly $\delta \mathbf{U}=\mathbf{U}_{2}-\mathbf{U}_{1}=0$, see [101, 1, 2, Eq. (1, 1b) allows for an infinitesimal $\delta U \propto \delta x$, as can be seen from Eq. (6) of [53]. That means that [1 1b) holds for infinitesimally close curves belonging to an arbitrary geodesic congruence (it is in this sense that in e.g. [52, 100] $\delta U$ is portrayed as "arbitrary" - it is understood to be infinitesimal therein, as those treatments deal with congruences of curves).
} 
equations, consisting of two parts: i) a subset of four equations formally very similar to Maxwell's, that are likewise algebraic equations involving only tidal tensors and sources (the mass-energy current vector), and ii) a fifth equation involving the space part of $T^{\alpha \beta}$ and a spatial rank 2 tensor which has no electromagnetic analogue. This is what we are going to show next. For that, we first introduce the time and space projectors with respect to a unit time-like vector $U^{\alpha}$ (i.e., the projectors parallel and orthogonal to $\left.U^{\alpha}\right)$ :

$$
\top_{\beta}^{\alpha} \equiv\left(\top^{U}\right)_{\beta}^{\alpha}=-U^{\alpha} U_{\beta} ; \quad h_{\beta}^{\alpha} \equiv\left(h^{U}\right)_{\beta}^{\alpha}=U^{\alpha} U_{\beta}+\delta_{\beta}^{\alpha} .
$$

A vector $A^{\alpha}$ can be split into its time and space projections with respect to $U^{\alpha}$; and an arbitrary rank $n$ tensor can be completely decomposed taking time and space projections in each of its indices (e.g. [27]):

$$
A^{\alpha}=\top_{\beta}^{\alpha} A^{\beta}+h_{\beta}^{\alpha} A^{\beta} ; \quad A^{\alpha_{1} \ldots \alpha_{n}}=\left(\top_{\beta_{1}}^{\alpha_{1}}+h_{\beta_{1}}^{\alpha_{1}}\right) \ldots\left(\top_{\beta_{n}}^{\alpha_{n}}+h_{\beta_{n}}^{\alpha_{n}}\right) A^{\beta_{1} \ldots \beta_{n}} .
$$

Instead of using $h_{\sigma}^{\mu}$, one can also, if convenient, spatially project an index of a tensor $A^{\sigma \ldots}$ contracting it with the spatial 3-form $\epsilon_{\alpha \beta \sigma \gamma} U^{\gamma}$; for instance, for the case of vector $A^{\sigma}$, one obtains the spatial 2-form $\epsilon_{\alpha \beta \sigma \gamma} U^{\gamma} A^{\sigma}=\star A_{\alpha \beta \gamma} U^{\gamma}$, which contains precisely the same information as the spatial vector $A^{\mu} h^{\sigma}{ }_{\mu} \equiv A^{\langle\sigma\rangle}$ (the former is the spatial dual of the latter). New contraction with $\epsilon_{\mu \nu}^{\alpha \beta} U^{\nu}$ yields $A^{\langle\sigma\rangle}$ again. Indeed we may write

$$
h_{\sigma}^{\mu}=\frac{1}{2} \epsilon^{\alpha \beta \mu \nu} U_{\nu} \epsilon_{\alpha \beta \sigma \gamma} U^{\gamma} .
$$

Another very useful relation is the following. The space projection $h^{\mu}{ }_{\alpha} h_{\beta}^{\nu} F_{\mu \nu} \equiv F_{\langle\alpha\rangle\langle\beta\rangle}$ of a 2 -form $F_{\alpha \beta}=F_{[\alpha \beta]}$ is equivalent to the tensor $\epsilon_{\mu \nu \alpha \beta} F^{\alpha \beta} U^{\nu}=2 \star F_{\mu \nu} U^{\nu}$ (i.e., spatially projecting $F_{\alpha \beta}$ is equivalent to time-projecting its Hodge dual). We have:

$$
F_{\langle\alpha\rangle\langle\beta\rangle}=\frac{1}{2} \epsilon_{\mu \alpha \beta \lambda} U^{\lambda} \epsilon_{\nu \sigma \delta}^{\mu} U^{\nu} F^{\sigma \delta}=\epsilon_{\mu \alpha \beta \lambda} U^{\lambda} \star F_{\nu}^{\mu} U^{\nu} .
$$

Now let $F_{\gamma_{1} \ldots \gamma_{n} \alpha \beta \delta_{1} \ldots \delta_{m}}=F_{\gamma_{1} \ldots \gamma_{n}[\alpha \beta] \delta_{1} \ldots \delta_{m}}$, be some tensor antisymmetric in the pair $\alpha, \beta$; an equality similar to the one above applies:

$$
F_{\gamma_{1} \ldots \gamma_{n}\langle\alpha\rangle\langle\beta\rangle \delta_{1} \ldots \delta_{m}}=\frac{1}{2} \epsilon_{\mu \alpha \beta \lambda} U^{\lambda} \epsilon_{\nu \sigma \delta}^{\mu} U^{\nu} F_{\gamma_{1} \ldots \gamma_{n}}{ }^{\sigma \delta}{ }_{\delta_{1} \ldots \delta_{m}} .
$$

\subsubsection{Maxwell's equations}

Maxwell's equations are given in tensor form by the pair of equations:

$$
F_{; \beta}^{\alpha \beta}=4 \pi j^{\alpha} \quad(\mathrm{a}) ; \quad \star F_{; \beta}^{\alpha \beta}=0 \quad(\mathrm{~b}) .
$$

Here (6a) are the Maxwell source equations, and (6b) are the source-free equations, equivalent to $F_{[\alpha \beta ; \gamma]}=0$, and commonly called the Bianchi identity for $F_{\alpha \beta} . j^{\alpha}$ denotes the current 4-vector. Decomposing the tensors $F_{\alpha \beta ; \gamma}$ and $\star F_{\alpha \beta ; \gamma}$ into their time and space projections in the first two indices, using Eq. (4) to project spatially, we obtain their decomposition in terms of tidal tensors,

$$
\begin{aligned}
F_{\alpha \beta ; \gamma} & =2 U_{[\alpha} E_{\beta] \gamma}+\epsilon_{\alpha \beta \mu \sigma} U^{\sigma} B_{\gamma}^{\mu} ; \\
\star F_{\alpha \beta ; \gamma} & =2 U_{[\alpha} B_{\beta] \gamma}-\epsilon_{\alpha \beta \mu \sigma} U^{\sigma} E_{\gamma}^{\mu} .
\end{aligned}
$$


Substituting these decompositions into Eqs. (6) and taking time and space projections, we obtain the set of four equations:

$$
\begin{aligned}
E_{\alpha}^{\alpha} & =4 \pi \rho_{c} ; \\
E_{[\alpha \beta]} & =U_{[\alpha} E_{\beta] \gamma} U^{\gamma}+\frac{1}{2} \epsilon_{\alpha \beta \mu \sigma} U^{\sigma} B^{\mu \gamma} U_{\gamma} ; \\
B_{\alpha}^{\alpha} & =0 ; \\
B_{[\alpha \beta]} & =U_{[\alpha} B_{\beta] \gamma} U^{\gamma}-\frac{1}{2} \epsilon_{\alpha \beta \mu \sigma} U^{\sigma} E^{\mu \gamma} U_{\gamma}-2 \pi \epsilon_{\alpha \beta \sigma \gamma} j^{\sigma} U^{\gamma} .
\end{aligned}
$$

Here $\rho_{c} \equiv-j^{\alpha} U_{\alpha}$ is the charge density as measured by an observer of 4-velocity $U^{\alpha}$. In more detail, taking the time projection of (6a), we obtain Eq. (9); taking the space projection, by contracting with the spatial 3-form $\epsilon_{\mu \nu \alpha \sigma} U^{\sigma}$, yields Eq. (12). The same procedure applied to Eq. (6p) yields Eqs. (11) and (10) as time and space projections, respectively.

Hence, in this formalism, Maxwell's equations are cast as the equations for the traces and antisymmetric parts of the electromagnetic tidal tensors; and they involve only tidal tensors and sources. Substituting (7)-(8) back into (10) and (12), leads to the equivalent set Eqs. (1, 4a)-(1,8a) of Table 1. It is also useful to note that the pair of Eqs. 10 and $(12$ ) can be condensed into the equivalent pair

$$
\epsilon_{\alpha \delta}^{\beta \gamma} U^{\delta} E_{[\gamma \beta]}=-B_{\alpha \beta} U^{\beta} ; \quad \text { (a) } \quad \epsilon_{\alpha \delta}^{\beta \gamma} U^{\delta} B_{[\gamma \beta]}=E_{\alpha \beta} U^{\beta}+4 \pi j_{\alpha} .
$$

In a Lorentz frame in flat spacetime, since $U_{; \beta}^{\alpha}=U_{, \beta}^{\alpha}=0$, we have $E_{\gamma \beta}=E_{\gamma ; \beta}, B_{\gamma \beta}=B_{\gamma ; \beta}$; and (using $U^{\alpha}=\delta_{0}^{\alpha}$ ) Eqs. 13 can be written in the familiar vector forms $\nabla \times \vec{E}=-\partial \vec{B} / \partial t$ and $\nabla \times \vec{B}=\partial \vec{E} / \partial t+4 \pi \vec{j}$, respectively. Likewise, Eqs. (9) and 11 reduce in this frame to the familiar forms $\nabla \cdot \vec{E}=4 \pi \rho_{c}$ and $\nabla \cdot \vec{B}=0$, respectively.

\subsubsection{Einstein's equations}

Equations (14a) below are the Einstein source equations for the gravitational field; Eqs (14b) are the algebraic Bianchi identity, equivalent to $R_{[\alpha \beta \gamma] \delta}=0$ :

$$
R_{\alpha \gamma \beta}^{\gamma} \equiv R_{\alpha \beta}=8 \pi\left(T_{\alpha \beta}-\frac{1}{2} g_{\alpha \beta} T_{\gamma}^{\gamma}\right) \quad(\mathrm{a}) ; \quad \star R_{\gamma \beta}^{\gamma \alpha}=0 \quad(\mathrm{~b}) .
$$

In order to express these equations in the tidal tensor formalism we will decompose the Riemann tensor into its time and space projections (in each of its indices) with respect to a unit time-like vector $U^{\alpha}, R^{\alpha \beta \gamma \delta}=\left(\top_{\rho}^{\alpha}+h_{\rho}^{\alpha}\right) . .\left(T_{\sigma}^{\delta}+h_{\sigma}^{\delta}\right) R^{\rho . \sigma}$, cf. Eq. [3]; we obtain 2

$$
\begin{aligned}
R_{\gamma \delta}^{\alpha \beta}= & 4 \mathbb{E}_{[\gamma}^{[\alpha} U_{\delta]} U^{\beta]}+2\left\{\epsilon_{\gamma \delta}^{\mu \chi} U_{\chi} \mathbb{H}_{\mu}^{[\beta} U^{\alpha]}+\epsilon^{\mu \alpha \beta \chi} U_{\chi} \mathbb{H}_{\mu[\delta} U_{\gamma]}\right\} \\
& +\epsilon^{\alpha \beta \phi \psi} U_{\psi} \epsilon^{\mu \nu}{ }_{\gamma \delta} U_{\nu} \mathbb{F}_{\phi \mu},
\end{aligned}
$$

where we made use of the identity (4) to project spatially an antisymmetric pair of indices, noting that $R_{\alpha \beta \gamma \delta}$ can be regarded as a double 2 -form. This equation tells us that the Riemann tensor

\footnotetext{
${ }^{2}$ The characterization of the Riemann tensor by these three spatial rank 2 tensors is known as the "Bel decomposition", even though the explicit decomposition (15) is not presented in any of Bel's papers (e.g. [5]). To the author's knowledge, an equivalent expression (Eq. (4.6) therein) can only be found at 91.
} 
decomposes, with respect to $U^{\alpha}$, into three spatial tensors: the gravitoelectric tidal tensor $\mathbb{E}_{\alpha \beta}$, the gravitomagnetic tidal tensor $\mathbb{H}_{\alpha \beta}$, plus a third tensor

$$
\mathbb{F}_{\alpha \beta} \equiv \star R \star_{\alpha \gamma \beta \delta} U^{\gamma} U^{\delta}=\epsilon_{\alpha \gamma}^{\mu \nu} \epsilon_{\beta \delta}^{\lambda \tau} R_{\mu \nu \lambda \tau} U^{\gamma} U^{\delta}
$$

introduced by Bel [5], which encodes the purely spatial curvature with respect to $U^{\alpha}$, and has no electromagnetic analogue. In order to obtain Eq. 15, we made use of the symmetries $R^{\alpha \beta \gamma \delta}=$ $R^{[\alpha \beta][\gamma \delta]}$, and in the case of the terms involving $\mathbb{H}_{\alpha \beta}$ (and only for these terms) we also assumed the pair exchange symmetry $R^{\alpha \beta \gamma \delta}=R^{\gamma \delta \alpha \beta} . \mathbb{E}_{\alpha \beta}$ and $\mathbb{F}_{\alpha \beta}$ are symmetric (and spatial), and therefore have 6 independent components each; $\mathbb{H}_{\alpha \beta}$ is traceless (and spatial), and so has 8 independent components. Therefore these three tensors together encode the 20 independent components of the Riemann tensor.

In what follows we will need also the Hodge dual, in the first two indices, of the decomposition (15):

$$
\begin{aligned}
\star R_{\gamma \delta}^{\alpha \beta}= & 2 \epsilon_{\lambda \tau}^{\alpha \beta} \mathbb{E}_{[\gamma}^{\lambda} U_{\delta]} U^{\tau}+4 U^{[\alpha} \mathbb{H}_{[\delta}^{\beta]} U_{\gamma]}+\epsilon_{\lambda \tau}^{\alpha \beta} \epsilon_{\gamma \delta}^{\mu \nu} U_{\nu} \mathbb{H}_{\mu}{ }^{\tau} U^{\lambda} \\
& -2 U^{[\alpha} \mathbb{F}^{\beta]}{ }_{\mu} \epsilon^{\mu \nu}{ }_{\gamma \delta} U_{\nu} .
\end{aligned}
$$

The Ricci tensor $R_{\delta}^{\beta}=R_{\alpha \delta}^{\alpha \beta}$ and the tensor $\star R_{\alpha \mu \beta}^{\mu}$ follow as:

$$
\begin{gathered}
R_{\delta}^{\beta}=-\epsilon^{\alpha \beta \mu \nu} \mathbb{H}_{\mu \alpha} U_{\delta} U_{\nu}-\epsilon_{\alpha \delta \mu \nu} \mathbb{H}^{\mu \alpha} U^{\beta} U^{\nu}-\mathbb{F}_{\delta}^{\beta}-\mathbb{E}_{\delta}^{\beta}+\mathbb{E}_{\sigma}^{\sigma} U^{\beta} U_{\delta}+\mathbb{F}_{\sigma}^{\sigma} h_{\delta}^{\beta}, \\
\star R^{\alpha \beta}{ }_{\alpha \delta}=\epsilon_{\lambda \tau}^{\alpha \beta} \mathbb{E}_{\alpha}^{\lambda} U_{\delta} U^{\tau}-\delta^{\beta}{ }_{\delta} \mathbb{H}^{\alpha}{ }_{\alpha}+U^{\beta} \mathbb{F}_{\mu}^{\alpha} \epsilon_{\alpha \delta}^{\mu \nu} U_{\nu} .
\end{gathered}
$$

Substituting (17) into (14 $)$, and (18) into (14 $\mathrm{b})$, we obtain Einstein's equations and the algebraic Bianchi identities in terms of the tensors $\mathbb{E}_{\alpha \beta}, \mathbb{H}_{\alpha \beta}, \mathbb{F}_{\alpha \beta}$. Now let us make the time-space splitting of these equations. Eq. (14a) is symmetric, hence it only has 3 non-trivial projections: time-time, time-space, and space-space. The time-time projection yields

$$
\mathbb{E}_{\alpha}^{\alpha}=4 \pi\left(2 \rho+T_{\alpha}^{\alpha}\right)
$$

where $\rho \equiv T^{\alpha \beta} U_{\beta} U_{\alpha}$ denotes the mass-energy density as measured by an observer of 4-velocity $U^{\alpha}$. Contraction of (17) with the time-space projector $\top^{\theta}{ }_{\beta} \epsilon_{\sigma \tau \gamma}^{\delta} U^{\gamma}$ yields, using (14a):

$$
\mathbb{H}_{[\sigma \tau]}=-4 \pi \epsilon_{\lambda \sigma \tau \gamma} J^{\lambda} U^{\gamma}
$$

where $J^{\alpha} \equiv-T^{\alpha \beta} U_{\beta}$ is the mass/energy current as measured by an observer of 4 -velocity $U^{\alpha}$. The space-space projection yields:

$$
\mathbb{F}_{\theta}^{\lambda}+\mathbb{E}^{\lambda}{ }_{\theta}-\mathbb{F}_{\sigma}^{\sigma} h_{\theta}^{\lambda}=8 \pi\left[h^{\lambda} \frac{1}{2} T_{\alpha}^{\alpha}-T_{\langle\theta\rangle}^{\langle\lambda\rangle}\right]
$$

where $T_{\langle\theta\rangle}^{\langle\lambda\rangle} \equiv h^{\lambda}{ }_{\delta} h_{\theta}^{\beta} T_{\beta}^{\delta}$.

Since the tensor $\star R^{\gamma \alpha}{ }_{\gamma \beta}$ is not symmetric, Eq. $(14 \mathrm{p})$ seemingly splits into four parts: a timetime, time-space, space-time, and space-space projections. However, the time-time and space-space 
projections yield the same equation. Substituting decomposition (18) into Eq. (14) b), and taking the time-time (or space-space), time-space, and space-time projections, yields, respectively:

$$
\mathbb{H}_{\alpha}^{\alpha}=0 ; \quad \text { (a) } \quad \mathbb{F}_{[\alpha \beta]}=0 ; \quad \text { (b) } \quad \mathbb{E}_{[\alpha \beta]}=0 \quad \text { (c) }
$$

Note however that Eqs. (19)-(22) are not a set of six independent equations (only five), as Eqs. (22) $), 22$ c) and (21) are not independent; using the latter, together with $(22 \mathrm{~b}) /(22 \mathrm{c})$, one can obtain the remaining one, $(22 \mathrm{c}) /(22 \mathrm{~b})$.

The gravitational field equations are summarized and contrasted with their electromagnetic counterparts in Table 1. Eqs. (1.4b)-(1.5b), (1.7b)-(1,8b) are very similar in form to Maxwell Eqs. (1.4a)-(1,8a); they are their physical gravitational analogues, since both are the traces and antisymmetric parts of the tensors $\left\{E_{\alpha \beta}, B_{\alpha \beta}\right\} \leftrightarrow\left\{\mathbb{E}_{\alpha \beta}, \mathbb{H}_{\alpha \beta}\right\}$, which we know, from equations (1.1) and (1.2), to play analogous physical roles in the two theories. Note this interesting aspect of the analogy: if one replaces, in Eqs. (9)- $(12)$, the electromagnetic tidal tensors $\left(E_{\alpha \beta}\right.$ and $\left.B_{\alpha \beta}\right)$ by the gravitational ones $\left(\mathbb{E}_{\alpha \beta}\right.$ and $\left.\mathbb{H}_{\alpha \beta}\right)$, and the charges by masses (i.e., density $\rho_{c}$ and current $j^{\alpha}$ of charge by density $\rho$ and current $J^{\alpha}$ of mass), one almost obtains Eqs. (1.4b)-(1.5b), (1.7b)(1.8b), apart from a factor of 2 in the source term in $(1,5 \mathrm{~b})$ and the difference in the source of (1.4b). This happens because, since $\mathbb{E}_{\alpha \beta}$ and $\mathbb{H}_{\alpha \beta}$ are spatial tensors, all the contractions with $U^{\alpha}$ present in Eqs. 10 and 12 vanish. In the case of vacuum, the four gravitational equations which are analogous to Maxwell's are thus exactly obtained from the latter by simply replacing $\left\{E_{\alpha \beta}, B_{\alpha \beta}\right\} \rightarrow\left\{\mathbb{E}_{\alpha \beta}, \mathbb{H}_{\alpha \beta}\right\}$.

Eqs. (22b) and (21), involving $\mathbb{F}_{\alpha \beta}$, have no electromagnetic analogue. Eq. (21) involves also, as a source, the space-space part of the energy momentum tensor, $T^{\langle\alpha\rangle\langle\beta\rangle}$, which, unlike the energy current 4-vector $J^{\alpha}=-T^{\alpha \beta} U_{\beta}$ (analogous to the charge current 4-vector $j^{\alpha}$ ), has no electromagnetic counterpart. It is worth discussing this equation in some detail. It has a fundamental differenc ${ }^{3}$ with respect to the other gravitational field equations in Table 1, and with their electromagnetic analogues: the latter are algebraic equations involving only the traces and antisymmetric parts of the tidal tensors (or of $\mathbb{F}_{\alpha \beta}$ ), plus the source terms; they impose no condition on the symmetric parts. In electromagnetism, this is what allows the field to be dynamical, and waves to exist (their tidal tensors are described, in an inertial frame, by Eqs. (157)-(158) below); were there additional independent algebraic equations for the traceless symmetric part of the tidal tensors, and these fields would be fixed. But Eq. (21), by contrast, is an equation for the symmetric parts of the tensors $\mathbb{E}_{\alpha \beta}$ and $\mathbb{F}_{\alpha \beta}$. It can be split into two parts. Taking the trace, and using (19), one obtains the source equation for $\mathbb{F}_{\alpha \beta}$ :

$$
\mathbb{F}_{\sigma}^{\sigma}=8 \pi \rho
$$

substituting back into (21) we get:

$$
\mathbb{F}_{\beta}^{\alpha}+\mathbb{E}_{\beta}^{\alpha}=8 \pi\left[h_{\beta}^{\alpha}\left(\frac{1}{2} T_{\gamma}^{\gamma}+\rho\right)-T_{\langle\beta\rangle}^{\langle\alpha\rangle}\right] .
$$

This equation tells us that the tensor $\mathbb{F}_{\beta}^{\alpha}$ is not an extra (comparing with electrodynamics) independent object; given the sources and the gravitoelectric tidal tensor $\mathbb{E}_{\alpha \beta}, \mathbb{F}_{\alpha \beta}$ is completely determined by (24).

\footnotetext{
${ }^{3}$ We thank João Penedones for drawing our attention to this point.
} 
In vacuum $\left(T^{\alpha \beta}=0, j^{\alpha}=0\right)$, the Riemann tensor becomes the Weyl tensor: $R_{\alpha \beta \gamma \delta}=C_{\alpha \beta \gamma \delta}$; due to the self duality property of the latter: $C_{\alpha \beta \gamma \delta}=-\star C \star_{\alpha \beta \gamma \delta}$, it follows that $\mathbb{F}_{\alpha \beta}=-\mathbb{E}_{\alpha \beta}$.

The equations in Table 1 have the status of constraints for the tidal fields. They are especially suited to compare the tidal dynamics (i.e., Eqs. (1.1) and (1.2)) of the two interactions, which is discussed in the next section. But they do not tell us about the dynamics of the fields themselves. To obtain dynamical field equations, one possible route is to take one step back and express the tidal tensors in terms of gauge fields (such as the GEM inertial fields $\vec{G}, \vec{H}$ and the shear $K_{(\alpha \beta)}$ of the $1+3$ formalism of Sec. 3 the general expressions of Einstein equations in terms of these fields is given Sec. 3.4 .2 below; but is also possible to write the equations for the dynamics of the tidal tensors (the physical fields); that is done not through Einstein equations (14), but through the differential Bianchi identity $R_{\sigma \tau[\mu \nu ; \alpha]}=0$, together with decomposition (15), and using (14) to substitute $R^{\alpha \beta}$ by the source terms. The resulting equations, for the case of vacuum (where $\left\{\mathcal{E}_{\alpha \beta}, \mathcal{H}_{\alpha \beta}\right\}=\left\{\mathbb{E}_{\alpha \beta}, \mathbb{H}_{\alpha \beta}\right\}$ ), are Eqs. 150-151) of Sec. 6 below. One may write as well dynamical equations for the electromagnetic tidal tensors, which for the case of vacuum, and an inertial frame, are Eqs. (157)-158) of Sec. 6.1 however in the electromagnetic case the fundamental physical fields are the vectors $E^{\alpha}, B^{\alpha}$, whose covariant field equations are Eqs. 152)-(155) (the tidal field equations (157)-158) follow trivially from these).

\subsection{Gravity vs Electromagnetism}

In the tidal tensor formalism, cf. Table 1, the gravitational field is described by five (independent) algebraic equations, four of which analogous to the Maxwell equations, plus an additional equation, involving the tensor $\mathbb{F}_{\alpha \beta}$, which has no parallel in electromagnetism. Conversely, in Maxwell equations there are terms with no gravitational counterpart; these correspond to the antisymmetric parts / time projections (with respect to the observer congruence) of the electromagnetic tidal tensors.

The tensor $\mathbb{F}_{\alpha \beta}$ - whereas Maxwell's equations can be fully expressed in terms of tidal tensors and sources, the same is only true, in general, for the temporal part of Einstein's equations. The Space-Space part, Eq. (21), involves the tensor $\mathbb{F}_{\alpha \beta}$, which has no electromagnetic analogue. This tensor, however, is not an additional independent object, as it is completely determined via (21) given the sources and $\mathbb{E}_{\alpha \beta}$. In vacuum $\mathbb{F}_{\alpha \beta}=-\mathbb{E}_{\alpha \beta}$.

Sources - The source of the gravitational field is the rank two energy momentum tensor $T^{\alpha \beta}$, whereas the source of the electromagnetic field is the current 4-vector $j^{\alpha}$. Using the projectors (2) one can split $T^{\alpha \beta}=\rho U^{\alpha} U^{\beta}+2 U^{(\alpha} h_{\mu}^{\beta)} J^{\mu}+T^{\langle\alpha\rangle\langle\beta\rangle}$, and $j^{\alpha}=\rho_{c} U^{\alpha}+h^{\alpha}{ }_{\mu} j^{\mu}$. Eqs. 114) show that the source of $E_{\alpha \beta}$ is $\rho_{c}$, and its gravitational analogue, as the source of $\mathbb{E}_{\alpha \beta}$, is $2 \rho+T_{\alpha}^{\alpha}(\rho+3 p$ for a perfect fluid). The magnetic/gravitomagnetic tidal tensors are analogously sourced by the charge/mass-energy currents $j^{\langle\mu\rangle} / J^{\langle\mu\rangle}$, as shown by Eqs. (1.5). Note that, when the Maxwell tensor is covariantly constant along the observer's worldline, $\star F_{\alpha \beta ; \gamma} U^{\gamma}$ vanishes and equations (1.5a) and (1.5b) match up to a factor of 2, identifying $j^{\langle\mu\rangle} \leftrightarrow J^{\langle\mu\rangle}$. Eq. 23 shows that $\rho$ is the source of $\mathbb{F}_{\alpha \beta}$. Eq. 1 , 6 ), sourced by the space-space part $T^{\langle\alpha\rangle\langle\beta\rangle}$, as well as the contribution $T_{\alpha}^{\alpha}$ for (1.4b), manifest the well known fact that in gravity, by contrast with electromagnetism, pressure and stresses act as sources of the field.

Symmetries and time projections of tidal tensors - The gravitational and electromagnetic tidal tensors do not generically exhibit the same symmetries; moreover, the former tidal tensors 
are spatial, whereas the latter have a time projection (with respect to the observer measuring

Table 1: The gravito-electromagnetic analogy based on tidal tensors.

Electromagnetism

Worldline deviation:

$\frac{D^{2} \delta x^{\alpha}}{d \tau^{2}}=\frac{q}{m} E_{\beta}^{\alpha} \delta x^{\beta}, \quad E_{\beta}^{\alpha} \equiv F_{\mu ; \beta}^{\alpha} U^{\mu}$

Force on magnetic dipole:

$F_{E M}^{\beta}=B_{\alpha}^{\beta} \mu^{\alpha}, B_{\beta}^{\alpha} \equiv \star F_{\mu ; \beta}^{\alpha} U^{\mu}$

(1.2a)

1.1a)

$\frac{D^{2} \delta x^{\alpha}}{d \tau^{2}}=-\mathbb{E}_{\beta}^{\alpha} \delta x^{\beta}$

Gravity

Force on gyroscope:

Differential precession of magnetic dipoles:

$$
\delta \Omega_{\mathrm{EM}}^{i}=-\sigma B^{i}{ }_{\beta} \delta x^{\beta}
$$

(1.3a)

$F_{G}^{\beta}=-\mathbb{H}_{\alpha}{ }^{\beta} S^{\alpha}, \quad \mathbb{H}^{\alpha}{ }_{\beta} \equiv \star R_{\mu \beta \nu}^{\alpha} U^{\mu} U^{\nu}$
Differential precession of gyroscopes:

$$
\delta \Omega_{\mathrm{G}}^{i}=\mathbb{H}^{i}{ }_{\beta} \delta x^{\beta}
$$

Maxwell Source Equations

$$
F_{; \beta}^{\alpha \beta}=4 \pi j^{\alpha}
$$

- Time Projection:

$$
E_{\alpha}^{\alpha}=4 \pi \rho_{c}
$$

- Space Projection:

$B_{[\alpha \beta]}=\frac{1}{2} \star F_{\alpha \beta ; \gamma} U^{\gamma}-2 \pi \epsilon_{\alpha \beta \sigma \gamma} j^{\sigma} U^{\gamma}$

No electromagnetic analogue

Bianchi Identity

$\star F_{; \beta}^{\alpha \beta}=0 \quad\left(\Leftrightarrow F_{[\alpha \beta ; \gamma]}=0\right)$

- Time Projection:

$$
B_{\alpha}^{\alpha}=0
$$

- Space Projection:

$$
E_{[\alpha \beta]}=\frac{1}{2} F_{\alpha \beta ; \gamma} U^{\gamma}
$$

No electromagnetic analogue
Einstein Equations

$R_{\mu \nu}=8 \pi\left(T_{\mu \nu}-\frac{1}{2} g_{\mu \nu} T_{\alpha}^{\alpha}\right)$

- Time-Time Projection:

$$
\mathbb{E}_{\alpha}^{\alpha}=4 \pi\left(2 \rho+T_{\alpha}^{\alpha}\right)
$$

- Time-Space Projection:

$\mathbb{H}_{[\alpha \beta]}=-4 \pi \epsilon_{\alpha \beta \sigma \gamma} J^{\sigma} U^{\gamma}$

- Space-Space Projection:

$$
\mathbb{F}_{\beta}^{\alpha}+\mathbb{E}_{\beta}^{\alpha}-\mathbb{F}_{\sigma}^{\sigma} h_{\beta}^{\alpha}=8 \pi\left[\frac{1}{2} T_{\gamma}^{\gamma} h_{\beta}^{\alpha}-T_{\langle\beta\rangle}^{\langle\alpha\rangle}\right]
$$

Algebraic Bianchi Identity

$$
\star R_{\gamma \beta}^{\gamma \alpha}=0 \quad\left(\Leftrightarrow R_{[\alpha \beta \gamma] \delta}=0\right)
$$

- Time-Time (or Space-Space) Proj.:

$$
\mathbb{H}^{\alpha}{ }_{\alpha}=0
$$

- Space-Time Projection:

$$
\mathbb{E}_{[\alpha \beta]}=0
$$

- Time-Space Projection:

$$
\mathbb{F}_{[\alpha \beta]}=0
$$

them), signaling fundamental differences between the two interactions. In the general case of fields that vary along the observer's worldline (that is the case of an intrinsically non-stationary field, or an observer moving in a stationary non-uniform field), $E_{\alpha \beta}$ possesses an antisymmetric part; $\mathbb{E}_{\alpha \beta}$, by contrast, is always symmetric. $E_{[\alpha \beta]}$ encodes Faraday's law of induction: as discussed above, $E_{\alpha \beta}$ is a covariant derivative of the electric field as measured in the momentarily comoving reference frame (MCRF); thus Eq. (13a) is a covariant way of writing the Maxwell-Faraday equation $\nabla \times \vec{E}=-\partial \vec{B} / \partial t$. Therefore, the statement encoded in the equation $\mathbb{E}_{[\alpha \beta]}=0$ is that there are no 
analogous induction effects in the physical (i.e., tidal) gravitational forces (in the language of GEM vector fields of Sec. 3, we can say that the curl of the gravitoelectric field $\vec{G}$ does not manifest itself in the tidal forces, unlike its electromagnetic counterpart; see Sec. 3.5 for explicit demonstration). To see a physical consequence, let $\delta x^{\alpha}$ in Eq. (1.1a) - the separation vector between a pair of particles with the same $q / m$ and the same 4 -velocity $U^{\alpha}$ - be spatial with respect to $U^{\alpha}\left(\delta x^{\alpha} U_{\alpha}=0\right)$; and note that the spatially projected antisymmetric part of $E_{\mu \nu}$ can be written in terms of the dual spatial vector $\alpha^{\mu}: E_{[\langle\mu\rangle\langle\nu\rangle]}=\epsilon_{\mu \nu \gamma \delta} \alpha^{\gamma} U^{\delta}$. Then the spatial components (1.1a) can be written as (using $\left.E_{\langle\mu\rangle\langle\nu\rangle}=E_{(\langle\mu\rangle\langle\nu\rangle)}+E_{[\langle\mu\rangle\langle\nu\rangle]}\right)$ :

$$
\frac{D^{2} \delta x_{\langle\mu\rangle}}{d \tau^{2}}=\frac{q}{m}\left[E_{(\langle\mu\rangle\langle\nu\rangle)} \delta x^{\nu}+\epsilon_{\mu \nu \gamma \delta} \alpha^{\gamma} U^{\delta} \delta x^{\nu}\right] \quad \Leftrightarrow \quad \frac{D^{2} \delta \vec{x}}{d \tau^{2}}=\frac{q}{m}[\overleftrightarrow{E} \cdot \delta \vec{x}+\delta \vec{x} \times \vec{\alpha}],
$$

the second equation holding in the frame $U^{i}=0$, where we used the dyadic notation $\overleftrightarrow{E}$ of e.g. [89. From the form of the second equation we see that $q \vec{\alpha} / m$ is minus an angular acceleration. Using equation $\sqrt{13}$ ), we see that $\alpha^{\mu}=B^{\mu}{ }_{\beta} U^{\beta}$; and in an inertial frame $\vec{\alpha}=\partial \vec{B} / \partial t=-\nabla \times \vec{E}$. In the gravitational case, since $\mathbb{E}_{\mu \nu}=\mathbb{E}_{(\mu \nu)}=\mathbb{E}_{\langle\mu\rangle\langle\nu\rangle}$, we have

$$
\frac{D^{2} \delta x_{\langle\mu\rangle}}{d \tau^{2}}=\frac{D^{2} \delta x_{\mu}}{d \tau^{2}}=-\mathbb{E}_{(\mu \nu)} \delta x^{\nu} \quad \Leftrightarrow \quad \frac{D^{2} \delta \vec{x}}{d \tau^{2}}=-\overleftrightarrow{\mathbb{E}} \cdot \delta \vec{x}
$$

That is, given a set of neighboring charged test particles, the electromagnetic field "shears" the set via $E_{(\mu \nu)}$, and induces an accelerated rotation ${ }^{4}$ via the laws of electromagnetic induction encoded in $E_{[\mu \nu]}$. The gravitational field, by contrast, only shears ${ }^{5}$ the set, since $\mathbb{E}_{[\mu \nu]}=0$.

Further physical evidence for the absence of a gravitational analogue for Faraday's law of induction in the physical forces and torques is given in the companion paper [6]: consider a spinning spherical charged body in an electromagnetic field; and choose the MCRF; if the magnetic field is not constant in this frame, by virtue of equation $\nabla \times \vec{E}=-\partial \vec{B} / \partial t$, a torque will in general be exerted on the body by the induced electric field, changing its angular momentum and kinetic energy of rotation. By contrast, no gravitational torque is exerted on a spinning "spherical" body (i.e., a particle whose multipole moments in a local orthonormal frame match the ones of a spherical body in flat spacetime) placed in an arbitrary gravitational field; its angular momentum and kinetic energy of rotation are constant.

As discussed in the previous section, the symmetry of $\mathbb{E}_{\alpha \beta}$ follows from the algebraic Bianchi identity $R_{[\beta \gamma \delta]}^{\alpha}=0$; this identity states that $R_{\beta \gamma \delta}^{\alpha}$ is the curvature tensor of a connection with vanishing torsion (the Levi-Civita connection of the space-time manifold). So one can say that the absence of electromagnetic-like induction effects is the statement that the physical gravitational forces are described by the curvature tensor of a connection without torsion.

\footnotetext{
${ }^{4}$ By rotation we mean here absolute rotation, i.e, measured with respect to a comoving Fermi-Walker transported frame. As one can check from the connection coefficients 39 below, in such frame $\left(\Omega_{\alpha \beta}=0\right)$ we have $d^{2} \delta x^{\hat{i}} / d \tau^{2}=$ $D^{2} \delta x^{\hat{i}} / d \tau^{2}$. See also in this respect [112].

${ }^{5}$ If the two particles were connected by a "rigid" rod then the symmetric part of the electric tidal tensor would also, in general, torque the rod; hence in such system we would have a rotation even in the gravitational case, see [25] pp. 154-155. The same is true for a quasi-rigid extended body; however, even in this case the effects due to the symmetric part are very different from the ones arising from electromagnetic induction: first, the former do not require the fields to vary along the particle's worldline, they exist even if the body is at rest in a stationary field; second, they vanish if the body is spherical, which does not happen with the torque generated by the induced electric field, see [6].
} 
There is also an antisymmetric contribution $\star F_{\alpha \beta ; \gamma} U^{\gamma}$ to $B_{\alpha \beta}$; in vacuum, Eq. (1.5a) is a covariant form of $\nabla \times \vec{B}=\partial \vec{E} / \partial t$; hence, the fact that, in vacuum, $\mathbb{H}_{[\alpha \beta]}=0$, means that there is no gravitational analogue to the antisymmetric part $B_{[\alpha \beta]}$ (i.e., the curl of $\vec{B}$ ) induced by the time varying field $\vec{E}$. Some physical consequences of this fact are explored in [6]: Eq. 11.5a) implies, via (1.2a), that whenever a magnetic dipole moves in a non-homogeneous field, it measures a nonvanishing $B_{[\alpha \beta]}$ (thus also $B_{\alpha \beta} \neq 0$ ), and therefore (except for very special orientations of the dipole moment $\mu^{\alpha}$ ) a force will be exerted on it; in the gravitational case, by contrast, the gravitational force on a gyroscope is not constrained to be non-vanishing when it moves in a non-homogeneous field; it is found that it may actually move along geodesics, as is the case of radial motion in Schwarzschild spacetim $£^{6}$, or circular geodesics in Kerr-dS spacetime.

The spatial character of the gravitational tidal tensors, contrasting with their electromagnetic counterparts, is another difference in the tensorial structure related to the laws of electromagnetic induction: as can be seen from Eqs. $(10)$ and $(12)$, the antisymmetric parts of $E_{\alpha \beta}$ and $B_{\alpha \beta}$ (in vacuum, for the latter) consist of time projections of these tidal tensors. Physically, these time projections are manifest for instance in the fact that the electromagnetic force on a magnetic dipole has a non-vanishing projection along the particle's 4-velocity $U^{\alpha}$, which is the rate of work done on it by the induced electric field [1, 6], and is reflected in a variation of the particle's proper mass. The projection, along $U^{\alpha}$, of the gravitational force (1.2b), in turn, vanishes, and the gyroscope's proper mass is constant.

\subsection{The analogy for differential precession}

Eqs. (1.2) in Table 1 give $B_{\alpha \beta}$ and $\mathbb{H}_{\alpha \beta}$ a physical interpretation as the tensors which, when contracted with a magnetic/gravitomagnetic dipole vector, yield the force exerted on magnetic dipoles/gyroscopes. We will now show that these tensors can also be interpreted as tensors of "relative", or "differential", precession for these test particles; i.e., tensors that, when contracted with a separation vector $\delta x^{\beta}$, yield the angular velocity of precession of a spinning particle at a point $\mathcal{P}_{2}$ relative to a system of axes anchored to spinning particles, with the same 4-velocity (and the same gyromagnetic ratio $\sigma$, if an electromagnetic field is present), at the infinitesimally close point $\mathcal{P}_{1}$. This is analogous to the electric tidal tensors $E_{\alpha \beta}$ and $\mathbb{E}_{\alpha \beta}$, which, when contracted with $\delta x^{\beta}$, yield the relative acceleration of two infinitesimally close test particles with the same 4-velocity (and the same ratio $q / m$ in the electromagnetic case).

For clarity we will treat the gravitational and electromagnetic interactions separately. We will start by the gravitational problem. Our goal is to compute the precession of a gyroscope at some point $\mathcal{P}_{2}$ relative to a frame attached to guiding gyroscopes at the neighboring point $\mathcal{P}_{1}$. Let $U^{\alpha}$ be the 4 -velocity of the gyroscope, and $a^{\alpha}=D U^{\alpha} / d \tau$ its acceleration. According to the Mathisson-Papapetrou equations [47, 49, no torque is exerted on a gyroscope (taken as a poledipole particle) in a gravitational field; if one moreover assumes the Mathisson-Pirani [47, 50] spin condition $S^{\alpha \beta} U_{\beta}=0$, it follows from these equations that its spin vector undergoes Fermi-Walker

\footnotetext{
${ }^{6}$ This example is particularly interesting in this discussion. In the electromagnetic analogous problem, a magnetic dipole in (initially) radial motion in the Coulomb field of a point charge experiences a force; that force, as shown in [6], comes entirely from the antisymmetric part of the magnetic tidal tensor, $B_{\alpha \beta}=B_{[\alpha \beta]}$; it is thus a natural realization of the arguments above that $\mathbb{H}_{\alpha \beta}=0$ in the analogous gravitational problem.
} 
transport,

$$
\frac{D S^{\alpha}}{d \tau}=S_{\nu} a^{\nu} U^{\alpha}
$$

(for more details, see [6]; in the comoving frame, the spatial part reads $D \vec{S} / d \tau=0$ ). Thus the frame we are looking for is a tetrad Fermi-Walker transported along the worldline $L$ of the set of gyroscopes 1 (passing trough the location $\mathcal{P}_{1}$ ). There is a locally rectangular coordinate system associated to such tetrad, the so-called 7 "Fermi coordinates"; let $\mathbf{e}_{\alpha}$ denote its basis vectors and $\Gamma_{\beta \gamma}^{\alpha}$ its Christoffel symbols, $\Gamma_{\beta \gamma}^{\alpha} \mathbf{e}_{\alpha}=\nabla_{\mathbf{e}_{\beta}} \mathbf{e}_{\gamma}$. The vectors $\mathbf{e}_{\alpha}$ are Fermi-Walker transported along $L$, so $\left.\left\langle\nabla_{\mathbf{e}_{0}} \mathbf{e}_{i}, \mathbf{e}_{j}\right\rangle\right|_{\mathcal{P}_{1}}=0 \Rightarrow \Gamma_{0 i}^{j}\left(\mathcal{P}_{1}\right)=0$. Hence, a gyroscope at $\mathcal{P}_{1}$, momentarily at rest in this frame, by Eq. 27] obviously does not precess relative to it, $d \vec{S} /\left.d \tau\right|_{\mathcal{P}_{1}}=\left.\dot{\overrightarrow{\vec{S}}}\right|_{\mathcal{P}_{1}}=0$. Here the dot denotes ordinary derivative along $\mathbf{e}_{0}, \dot{A}^{\alpha} \equiv \partial_{0} A^{\alpha}$. However, outside $L$, the basis vectors $\mathbf{e}_{\alpha}$ are no longer Fermi-Walker transported, $\left.\left\langle\nabla_{\mathbf{e}_{0}} \mathbf{e}_{i}, \mathbf{e}_{j}\right\rangle\right|_{\mathcal{P}_{2}} \neq 0 \Rightarrow \Gamma_{0 i}^{j}\left(\mathcal{P}_{2}\right) \neq 0$. That means that gyroscope 2 , at a point $\mathcal{P}_{2}$ (outside $L$ ), will be be seen to precess relative to the frame $\mathbf{e}_{\alpha}: d \vec{S}_{2} /\left.d \tau_{2}\right|_{\mathcal{P}_{2}} \neq 0$. If the gyroscope is at rest in this frame $\left(U_{2}^{i}=0\right)$, we have

$$
\left.\frac{d S_{2}^{i}}{d \tau_{2}}\right|_{\mathcal{P}_{2}}=-\Gamma_{0 j}^{i}\left(\mathcal{P}_{2}\right) S_{2}^{j} U_{2}^{0} .
$$

The Christoffel symbol $\Gamma_{0 j}^{i}\left(\mathcal{P}_{2}\right)$, to first order in $\delta x$, can be obtained from e.g. Eqs. (20) of [83] (making $\Omega_{i j}=0$ therein); it reads $\Gamma_{0 j}^{i}\left(\mathcal{P}_{2}\right)=R_{j k 0}^{i}\left(\mathcal{P}_{1}\right) \delta x^{k}$. From Eq. (4) above, we note that

$$
R_{\langle\alpha\rangle\langle\beta\rangle \gamma \tau}=\epsilon_{\sigma \delta}^{\mu \nu} \epsilon_{\mu \alpha \beta \lambda} U^{\lambda} U_{\nu} R_{\gamma \tau}^{\sigma \delta}=\epsilon_{\mu \alpha \beta \lambda} U^{\lambda} U_{\nu} \star R_{\gamma \tau}^{\mu \nu},
$$

which, in the Fermi frame $\mathbf{e}_{\alpha}$ (orthonormal at $\mathcal{P}_{1}$ ), reads: $R_{i j \gamma \tau}=\epsilon_{i j k} \star R_{0 \gamma \tau}^{k}$. We thus have

$$
\Gamma_{0 j}^{i}\left(\mathcal{P}_{2}\right)=\epsilon_{j k}^{i} \star R_{0 l 0}^{k}\left(\mathcal{P}_{1}\right) \delta x^{l}=\epsilon^{i}{ }_{j k} \mathbb{H}^{k}{ }_{l}^{k} \delta x^{l} .
$$

$\Gamma_{0 j}^{i}\left(\mathcal{P}_{2}\right)$ is an antisymmetric matrix, which we can write as $\Gamma_{0 j}^{i}\left(\mathcal{P}_{2}\right)=\epsilon^{i}{ }_{j k} \delta \Omega_{\mathrm{G}}^{k}$, where

$$
\delta \Omega_{\mathrm{G}}^{i} \equiv \mathbb{H}^{i}{ }_{l} \delta x^{l}
$$

Substituting into 28, and noting that $U_{2}^{0}=\left(-g_{00}\right)^{-1 / 2}=1+\mathcal{O}\left(\delta x^{2}\right)$, we have, to first order in $\delta x$

$$
\left.\frac{d \vec{S}_{2}}{d \tau_{2}}\right|_{\mathcal{P}_{2}}=\left.\dot{\vec{S}}_{2}\right|_{\mathcal{P}_{2}}=\delta \vec{\Omega}_{\mathrm{G}} \times \vec{S}_{2},
$$

where in the first equality we noted that $d \vec{S}_{2} /\left.d \tau_{2}\right|_{\mathcal{P}_{2}}=\left.\dot{\vec{S}}_{2}\right|_{\mathcal{P}_{2}}+\mathcal{O}\left(\delta x^{3}\right)$. Thus, $\delta \vec{\Omega}_{\mathrm{G}}$ is the angular velocity of precession of gyroscopes at $\mathcal{P}_{2}$ with respect to the Fermi frame $\mathbf{e}_{i}$, locked to the guiding gyroscopes at $\mathcal{P}_{1}$. Obviously, this is just minus the angular velocity of rotation of the basis vectors $\mathbf{e}_{i}$ relative to Fermi-Walker transport at $\mathcal{P}_{2}$. This result was first obtained in a recent work [4] through a different procedure; we believe the derivation above is more clear, and shows that one

\footnotetext{
${ }^{7}$ Following Synge [82, by Fermi coordinates we mean the locally rectangular coordinate system associated to a tetrad Fermi-Walker transported along a worldline (the curve being the origin of the frame, and its tangent the time axis). Note the existence of different conventions in the literature: the so-called "Fermi normal coordinates" of e.g. [84, 52 are a special case of our definition, for the case that the worldline is geodesic. The "Fermi coordinates" of 83, in turn, are a generalization of our definition, for the case that the tetrad is not Fermi-Walker transported.
} 
of the assumptions made in [4] to obtain $\delta \vec{\Omega}_{\mathrm{G}}$ - that the gyroscopes at $\mathcal{P}_{1}$ and $\mathcal{P}_{2}$ have the same acceleration - is not necessary; in order for (30) to hold, they only need to be momentarily comoving 8 , in the sense that they have zero 3 -velocity in the Fermi coordinate system of $\mathcal{P}_{1}$. It is also worth noting that if the basis worldline $L$ through $\mathcal{P}_{1}$ is geodesic, then (30) still holds when the gyroscopes at $\mathcal{P}_{2}$ have an infinitesimal velocity $v \propto \delta x$ in such frame (as is straightforward to check by a similar computation for the case $U_{2}^{i} \neq 0$ ). Hence Eq. (30) applies to gyroscopes carried by infinitesimally close observers belonging to an arbitrary geodesic congruence (in a certain analogy with the geodesic deviation equation (1.1b) of Table 1). The differential dragging effect in terms of the eigenvectors of $\mathbb{H}_{\alpha \beta}$ and their associated field lines, as well as their visualization in different spacetimes, are discussed in detail in [4].

Let us turn now to the analogous electromagnetic problem. Consider, in flat spacetime, a triad of orthogonal magnetic dipoles (magnetic moment $\mu^{\alpha}=\sigma S^{\alpha}$ ), with the same gyromagnetic ratio $\sigma$ (so that they all precess with the same frequency), moving along a worldline $L$ of tangent $\mathbf{U}$ passing through $\mathcal{P}_{1}$. If the Mathisson-Pirani condition holds, the spin vector of a magnetic dipole evolves along $L$ as (e.g. [6]):

$$
\frac{D S^{\mu}}{d \tau}=S_{\nu} a^{\nu} U^{\mu}+\sigma \epsilon_{\alpha \beta \nu}^{\mu} U^{\nu} S^{\alpha} B^{\beta}
$$

where $B^{\alpha} \equiv \star F^{\alpha \beta} U_{\beta}$. The second term marks an obvious difference with the case of the gyroscope, as it means that magnetic dipoles do precess (with angular velocity $\Omega^{\alpha}=-\sigma B^{\alpha}$ ) with respect to the comoving Fermi-Walker transported frame. We shall compare this precession for dipoles at infinitesimally close points, and relate with the gravitational analogue. In the Fermi frame $\mathbf{e}_{\alpha}$ with origin at $L$, the space part of Eq. (31) reads (at $\mathcal{P}_{1}$ ),

$$
\left.\frac{d \vec{S}}{d \tau}\right|_{\mathcal{P}_{1}}=\vec{\Omega}\left(\mathcal{P}_{1}\right) \times \vec{S} ; \quad \vec{\Omega}\left(\mathcal{P}_{1}\right) \equiv-\sigma \vec{B}\left(\mathcal{P}_{1}\right) .
$$

Now consider a magnetic dipole at the neighboring point $\mathcal{P}_{2}$, at rest in the frame $\mathbf{e}_{\alpha}$ (i.e. $U_{2}^{i}=0$, so that we are using the same notion of comoving as in the gravitational case); we have

$$
\left.\frac{d S_{2}^{i}}{d \tau_{2}}\right|_{\mathcal{P}_{2}}=-\Gamma_{0 j}^{i}\left(\mathcal{P}_{2}\right) S_{2}^{j}+\left(\vec{\Omega}\left(\mathcal{P}_{2}\right) \times \vec{S}_{2}\right)^{i}=\left(\vec{\Omega}\left(\mathcal{P}_{2}\right) \times \vec{S}_{2}\right)^{i} ; \quad \vec{\Omega}\left(\mathcal{P}_{2}\right) \equiv-\sigma \vec{B}\left(\mathcal{P}_{2}\right),
$$

where we noted that, in flat spacetime, $\Gamma_{0 j}^{i}=0$ everywhere in a Fermi frame (cf. Eqs (20) of [83]). Being at rest in the frame $\mathbf{e}_{\alpha}$ implies, in flat spacetims $9, \mathbf{U}_{2}=\mathbf{U}$ (note that parallelism between vectors at different points is well defined herein). Thus, $d S_{2}^{i} / d \tau_{2} \equiv\left(S_{2}\right)^{i}{ }_{\alpha} U_{2}^{\alpha}=d S_{2}^{i} / d \tau$, and $B^{\alpha}\left(\mathcal{P}_{2}\right) \equiv \star F_{\beta}^{\alpha}\left(\mathcal{P}_{2}\right) U_{2}^{\beta}=\star F_{\beta}^{\alpha}\left(\mathcal{P}_{2}\right) U^{\beta}$. Performing a Taylor expansion of $F_{\beta}^{\alpha}$ about $\mathcal{P}_{1}$ (and using for this operation a rectangular coordinate system, which one can always do in flat spacetime, so that $\left.F_{\beta, \gamma}^{\alpha}=F_{\beta ; \gamma}^{\alpha}\right)$, we may write $F_{\beta}^{\alpha}\left(\mathcal{P}_{2}\right)=F_{\beta}^{\alpha}\left(\mathcal{P}_{2}\right)+F_{\beta ; \gamma}^{\alpha}\left(\mathcal{P}_{1}\right) \delta x^{\gamma}+\mathcal{O}\left(\delta x^{2}\right)$. Therefore,

$$
B^{\alpha}\left(\mathcal{P}_{2}\right)=B^{\alpha}\left(\mathcal{P}_{1}\right)+B_{\gamma}^{\alpha}\left(\mathcal{P}_{1}\right) \delta x^{\gamma}+\mathcal{O}\left(\delta x^{2}\right)
$$

\footnotetext{
${ }^{8}$ The relative velocity of objects at different points is not a well defined notion in curved spacetime, since there is no natural way of comparing vectors at different points, see e.g. [85, 86. The notion of relative velocity implied above is dubbed in 85 . "Fermi relative velocity".

${ }^{9}$ This can be easily seen from the fact that the triad $\mathbf{e}_{i}$ coincides with the basis vectors of a momentarily comoving inertial frame; thus $U_{2}^{i}=0$ implies that, in the inertial frame, $U_{2}^{\alpha}=(1, \overrightarrow{0})=U^{\alpha}$. This can also be seen from Eqs. (22)-(23) of 85], to which we refer for a detailed discussion of the Fermi relative velocity in flat spacetime.
} 
where $B_{\alpha \beta}=\star F_{\alpha \gamma ; \beta} U^{\gamma}$ is the magnetic tidal tensor as defined in Eq. (1.2a) of Table 1. Eqs. (32) and $(33)$ are precessions measured with respect to the same frame $\mathbf{e}_{\alpha}$; taking the difference $\delta \vec{\Omega}_{\mathrm{EM}}$, we obtain

$$
\delta \Omega_{\mathrm{EM}}^{i}=\Omega^{i}\left(\mathcal{P}_{2}\right)-\Omega^{i}\left(\mathcal{P}_{1}\right)=-\sigma B_{\gamma}^{i} \delta x^{\gamma},
$$

which is analogous to Eq. (29), only with $-\sigma B_{\alpha \beta}$ in the place of $\mathbb{H}_{\alpha \beta}$.

It should be mentioned, however, that Eq. (35) does not, in general, yield the precession of dipole 2 with respect to a frame whose axes are anchored to the spin vectors of guiding magnetic dipoles at $\mathcal{P}_{1}$ (which would be perhaps the most natural analogue of the gravitational problem considered above). Let us denote by $\left(\mathbf{e}_{\text {dip }}\right)_{\alpha}$ the basis vectors of the coordinate system adapted to such frame (originating at $L$, where it is rectangular, with $\left(\mathbf{e}_{\text {dip }}\right)_{0}=\mathbf{U}$; this is a generalized version of the Fermi-coordinates of $L$, for the case that the spatial triad is not Fermi-Walker transported). The spin evolution equation for dipole 2 reads, in this frame,

$$
\left.\frac{d \vec{S}_{2}}{d \tau}\right|_{\mathcal{P}_{2}}=\delta \vec{\Omega}_{\mathrm{EM}} \times \vec{S}_{2}+\left(\vec{a} \times \vec{U}_{2}\right) \times \vec{S}_{2},
$$

where we used the connection coefficients given in Eqs. (20) of [83] (in particular, $\Gamma_{0 j}^{i}\left(\mathcal{P}_{2}\right)=\Omega^{i}{ }_{j}=$ $-\sigma \epsilon_{k j}^{i} B^{k}\left(\mathcal{P}_{1}\right)$ ), and noted that dipole 2 (since it is at rest in the Fermi frame $\mathbf{e}_{\alpha}$ ) moves in the frame $\left(\mathbf{e}_{\text {dip }}\right)_{\alpha}$ with spatial velocity $U_{2}^{i} \approx-\vec{\Omega}\left(\mathcal{P}_{1}\right) \times \vec{\delta} x$. Thus, only when $L$ is geodesic one has in such frame $d \vec{S}_{2} /\left.d \tau\right|_{\mathcal{P}_{2}}=\delta \vec{\Omega}_{\mathrm{EM}} \times \vec{S}_{2}$ (as for the acceleration of dipole 2, it can be arbitrary). It should also be noted that, by contrast with the gravitational Eqs. (29)-(30), Eqs. (35)-(36), do not hold when the dipoles possess an infinitesimal relative velocity $\delta U \propto \delta x$ (even if the basis worldline $L$ is geodesic, as an extra term $\star F_{\beta}^{\alpha} \delta U^{\beta}$ would show up in $\left.(34) ; \delta \mathbf{U}=\mathbf{U}_{2}-\mathbf{U}\right)$; they must be strictly comoving. This is analogous to the situation with the worldline deviation equations (1.1) of Table 1, where the gravitational equation allows the particles to have an infinitesimal deviation velocity, whereas the electromagnetic one does not (cf. footnote 1).

\section{Gravito-electromagnetic analogy based on inertial fields from the $1+3$ splitting of spacetime}

This approach has a different philosophy from the tidal tensor analogy of Sec. 2. Therein we aimed to compare physical, covariant forces of both theories; which was accomplished through the tidal forces. Herein the analogy drawn is between the electromagnetic fields $E^{\alpha}$, $B^{\alpha}$ and spatial inertial fields $G^{\alpha}, H^{\alpha}$ (i.e., fields of inertial forces, or "acceleration" fields), usually dubbed "gravitoelectromagnetic" (GEM) fields, that mimic $E^{\alpha}$ and $B^{\alpha}$ in gravitational dynamics. Inertial forces are fictitious forces, attached to a specific reference frame, and in this sense one can regard this analogy as a parallelism between physical forces from one theory, and reference frame effects from the other.

The GEM 3-vector fields are best known in the context of linearized theory for stationary spacetimes, e.g. [7, 11], where they are (somewhat naively) formulated as derivatives of the temporal components of the linearized metric tensor (the GEM potentials, in analogy with the EM potentials). More general approaches are possible if one observes that these are fields associated not to the local properties of a particular spacetime, but, as stated above, to the kinematical quantities of the 
reference frame. In particular, the GEM fields of the usual linearized approaches are but, up to some factors, the acceleration and vorticity of the congruence of zero 3 -velocity observers $\left(u^{\alpha} \simeq \delta_{0}^{\alpha}\right)$ in the chosen background. Taking this perspective, the GEM fields may actually be cast in an exact form, applying to arbitrary reference frames in arbitrary fields, through a general $1+3$ splitting of spacetime. In this section we present such an exact and general formulation. We take an arbitrary orthonormal reference frame, which can be thought as a continuous field of orthonormal tetrads, or, alternatively, as consisting of a congruence of observers, each of them carrying an orthonormal tetrad whose time axis is the observer's 4-velocity; the spatial triads, spanning the local rest space of the observers, are generically left arbitrary (namely their rotation with respect to Fermi-Walker transport). The inertial fields associated to this frame are, in this framework, encoded in the mixed time-space part of the connection coefficients: the acceleration $a^{\alpha}$ and vorticity $\omega^{\alpha}$ of the observer congruence, plus the rotation frequency $\Omega^{\alpha}$ of the spatial triads with respect to Fermi-Walker transport. The connection coefficients encode also the shear/expansion $K_{(\alpha \beta)}$ of the congruence. A "gravitoelectric" field is defined in this framework as $G^{\alpha} \equiv-a^{\alpha}$, and a gravitomagnetic field as $H^{\alpha} \equiv \Omega^{\alpha}+\omega^{\alpha}$; the motivation for these definitions being the geodesic equation, whose space part, in such frame, resembles the Lorentz force, with $G^{\alpha}$ in the role of an electric field, $H^{\alpha}$ in the role of a magnetic field, plus a third term with no electromagnetic analogue, involving $K_{(\alpha \beta)}$.

The treatment herein is to a large extent equivalent to what is dubbed in [27, 28, the "congruence point of view"; the main difference (apart from the differences in the formalism) is that we use a more general definition of gravitomagnetic field $H^{\alpha}$, allowing for an arbitrary rotation of the spatial frame (i.e., $\Omega^{\alpha}$ is left arbitrary), so that it adjusts to any frame. We also try to use a simplified notation, as the one in [27, 28], albeit very precise and rigorous, is not easy to follow. For that we work with orthonormal frames in most of our analysis, which are especially suited for our purposes because the connection coefficients associated to them are very simply related with the inertial fields. The price to pay is not having manifestly covariant equations at each step, by contrast with the formalism in [27, 28] (although the results are easily written in covariant form).

\subsection{The reference frame}

To an arbitrary observer moving along a worldline of tangent vector $u^{\alpha}$, one naturally associates an adapted local orthonormal frame (e.g. [27]), which is a tetrad $\mathbf{e}_{\hat{\alpha}}$ whose time axis is the observer's 4 -velocity, $\mathbf{e}_{\hat{0}}=\mathbf{u}$, and whose spatial triad $\mathbf{e}_{\hat{i}}$ spans the local rest space of the observer. The latter is for now undefined up to an arbitrary rotation. The evolution of the tetrad along the observer's worldline is generically described by the equation:

$$
\nabla_{\mathbf{u}} \mathbf{e}_{\hat{\beta}}=\Omega_{\hat{\beta}}^{\hat{\alpha}} \mathbf{e}_{\hat{\alpha}} ; \quad \Omega^{\alpha \beta}=2 u^{[\alpha} a^{\beta]}+\epsilon_{\nu \mu}^{\alpha \beta} \Omega^{\mu} u^{\nu}
$$

where $\Omega^{\alpha \beta}$ is the (anti-symmetric) infinitesimal generator of Lorentz transformations, whose spatial

part $\Omega_{\hat{i} \hat{j}}=\epsilon_{\hat{i} \hat{k} \hat{j}} \Omega^{\hat{k}}$ describes the arbitrary angular velocity $\vec{\Omega}$ of rotation of the spatial triad $\mathbf{e}_{\hat{i}}$ relative to a Fermi-Walker transported triad. $a^{\alpha} \equiv \nabla_{\mathbf{u}} u^{\alpha}$ is the observers' acceleration. Alternatively, from the definition of the connection coefficients,

$$
\nabla_{\mathbf{e}_{\hat{\beta}}} \mathbf{e}_{\hat{\gamma}}=\Gamma_{\hat{\beta} \hat{\gamma}}^{\hat{\alpha}} \mathbf{e}_{\hat{\alpha}}
$$




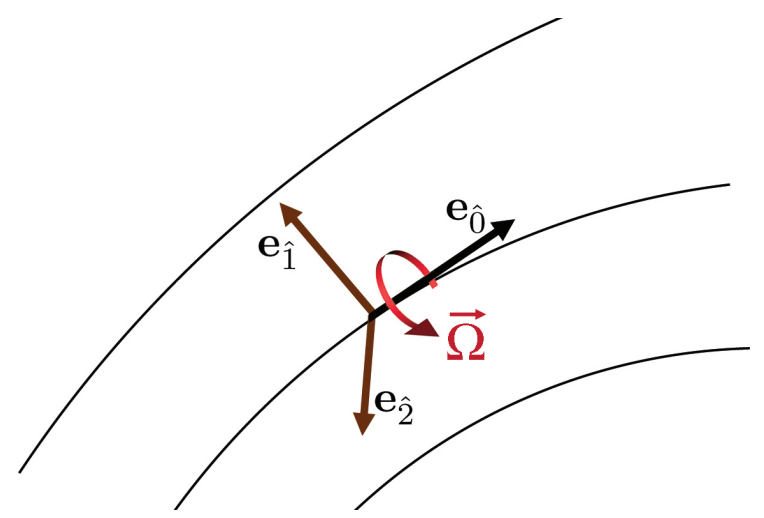

Figure 1: The reference frame: a congruence of of time-like curves — the observers' worldlines — of of tangent $\mathbf{u}$; each observer carries an orthonormal tetrad $\mathbf{e}_{\hat{\alpha}}$ such that $\mathbf{e}_{\hat{0}}=\mathbf{u}$ and the spatial triad $\mathbf{e}_{\hat{i}}$ spans the observer's local rest space. The triad $\mathbf{e}_{\hat{i}}$ rotates, relative to Fermi-Walker transport, with some prescribed angular velocity $\vec{\Omega}$.

we can think of the components of $\Omega^{\alpha \beta}$ as some of these coefficients:

$$
\begin{aligned}
& \Omega_{\hat{0}}^{\hat{i}}=\Gamma_{\hat{0} \hat{0}}^{\hat{i}}=\Gamma_{\hat{0} \hat{i}}^{\hat{0}}=a^{\hat{i}} ; \\
& \Omega_{\hat{j}}^{\hat{i}}=\Gamma_{\hat{0} \hat{j}}^{\hat{i}}=\epsilon_{\hat{i} \hat{k} \hat{j}} \Omega^{\hat{k}} .
\end{aligned}
$$

Unlike the situation in flat spacetime (and Lorentz coordinates), where one can take the tetrad adapted to a given inertial observer as a global frame, in the general case such tetrad is a valid frame only locally, in an infinitesimal neighborhood of the observer. In order to define a reference frame over an extended region of spacetime, one needs a congruence of observers, that is, one needs to extend $u^{\alpha}$ to a field of unit timelike vectors tangent to a congruence of time-like curves. A connecting vector $X^{\alpha}$ between two neighboring observers in the congruence satisfies

$$
[\mathbf{u}, \mathbf{X}]=\mathbf{0} \Leftrightarrow u^{\beta} \nabla_{\beta} X^{\alpha}-X^{\beta} \nabla_{\beta} u^{\alpha}=0 .
$$

The evolution of the connecting vector along the worldline of an observer in the congruence is then given by the linear equation

$$
\nabla_{\mathbf{u}} X^{\alpha}=\left(\nabla^{\beta} u^{\alpha}\right) X_{\beta}
$$

The component of the connecting vector orthogonal to the congruence,

$$
Y^{\alpha}=\left(h^{u}\right)_{\beta}^{\alpha} X^{\beta}=X^{\alpha}+\left(u_{\beta} X^{\beta}\right) u^{\alpha},
$$

satisfies

$$
\nabla_{\mathbf{u}} Y^{\alpha}=K^{\alpha \beta} Y_{\beta}+\left(a_{\beta} Y^{\beta}\right) u^{\alpha},
$$

where $K^{\alpha \beta}$ denotes the spatially projected covariant derivative of $u^{\alpha}$

$$
K^{\alpha \beta} \equiv\left(h^{u}\right)_{\lambda}^{\alpha}\left(h^{u}\right)_{\tau}^{\beta} u^{\lambda ; \tau}=\nabla^{\beta} u^{\alpha}+a^{\alpha} u^{\beta}
$$

The decomposition of this tensor into its trace, symmetric trace-free and anti-symmetric parts yields the expansion

$$
\theta=u_{; \alpha}^{\alpha}
$$


the shear

$$
\sigma_{\alpha \beta}=K_{(\alpha \beta)}-\frac{1}{3} \theta g_{\alpha \beta}-\frac{1}{3} \theta u_{\alpha} u_{\beta}
$$

and the vorticity

$$
\omega_{\alpha \beta}=K_{[\alpha \beta]}
$$

of the congruence. It is useful to introduce the vorticity vector

$$
\omega^{\alpha}=\frac{1}{2} \epsilon^{\alpha \beta \gamma \delta} u_{\gamma ; \beta} u_{\delta}=-\frac{1}{2} \epsilon^{\alpha \beta \gamma \delta} \omega_{\alpha \beta} u_{\delta} .
$$

According to definition above, $\omega^{\alpha}$ yields half the curl of $u^{\alpha}$; this is in agreement with the convention in e.g. [20, 27], but differs by a minus sign from the definition in e.g. [35, 36, 76]. Note however that for the vorticity tensor $\omega_{\alpha \beta}$ we are using the most usual definition given in [35, 36, 76], differing from a minus sign from the one in [27] (consequently, $\omega^{\alpha}$ given by Eq. (47) is minus the dual of $\left.\omega_{\alpha \beta}\right)$. The non-vanishing tetrad components of $K_{\alpha \beta}$ are

$$
K_{\hat{i} \hat{j}}=\sigma_{\hat{i} \hat{j}}+\frac{1}{3} \theta \delta_{\hat{i} \hat{j}}+\omega_{\hat{i} \hat{j}}
$$

These components determine the following connection coefficients:

$$
K_{\hat{i} \hat{j}}=\nabla_{\hat{j}} u_{\hat{i}}=\Gamma_{\hat{j} \hat{i}}^{\hat{0}}=\Gamma_{\hat{j} \hat{0}}^{\hat{i}} \text {. }
$$

The remaining temporal connection coefficients (other than the ones given in Eqs. (38)-(39), 449) above) are trivially zero:

$$
\Gamma_{\hat{\alpha} \hat{0}}^{\hat{0}}=-\mathbf{e}_{\hat{0}} \cdot \nabla_{\mathbf{e}_{\hat{\alpha}}} \mathbf{e}_{\hat{0}}=-\frac{1}{2} \nabla_{\mathbf{e}_{\hat{\alpha}}}\left(\mathbf{e}_{\hat{0}} \cdot \mathbf{e}_{\hat{0}}\right)=0 .
$$

Each observer in the congruence carries its own adapted tetrad, c.f. Fig. 1, and to define the reference frame one must provide the law of evolution for the spatial triads orthogonal to $u^{\alpha}$. A natural choice would be Fermi-Walker transport, $\vec{\Omega}=0$ (the triad does not rotate relative to local guiding gyroscopes); another natural choice, of great usefulness in this framework, is to lock the rotation of the spatial triads to the vorticity of the congruence, $\vec{\Omega}=\vec{\omega}$. We will dub such frame "congruence adapted frame' 10 , as argued in [56, 54] (see also [55]), this is the most natural generalization of the non-relativistic concept of reference frame; and the corresponding transport law $\vec{\Omega}=\vec{\omega}$ has been dubbed "co-rotating Fermi-Walker transport" [27, 28]. This choice is more intuitive in the special case of a shear-free congruence, where, as we will show next, the axes of the frame thereby defined point towards fixed neighboring observers. Indeed, if $X^{\alpha}$ is a connecting vector between two neighboring observers of the congruence and $Y^{\alpha}$ is its component orthogonal to the congruence, we have

$$
\nabla_{\mathbf{u}} Y^{\hat{i}}=\dot{Y}^{\hat{i}}+\Gamma_{\hat{0} \hat{0}}^{\hat{i}} Y^{\hat{0}}+\Gamma_{\hat{0} \hat{j}}^{\hat{i}} Y^{\hat{j}}=\dot{Y}^{\hat{i}}+\Omega_{\hat{j}}^{\hat{i}} X^{\hat{j}}
$$

\footnotetext{
${ }^{10}$ Note however that in some literature, e.g. 112, the term "congruence adapted" is employed with a different meaning, designating any tetrad field whose time axis is tangent to the congruence, without any restriction on the transport law for the spatial triad (namely without requiring the triads to co-rotate with the congruence). Hence "adapted" therein means what, in our convention, we would call adapted to each individual observer.
} 
where the dot denotes the ordinary derivative along $\mathbf{u}: \dot{A}_{\hat{\alpha}} \equiv A_{\hat{\alpha}, \hat{\beta}} u^{\hat{\beta}}$. Since, from $43, \nabla_{\mathbf{u}} Y^{\hat{i}}=$ $K_{\hat{j}}^{\hat{i}} Y^{\hat{j}}$, we conclude that

$$
\dot{Y}_{\hat{i}}=\left(\sigma_{\hat{i} \hat{j}}+\frac{1}{3} \theta \delta_{\hat{i} \hat{j}}+\omega_{\hat{i} \hat{j}}-\Omega_{\hat{i} \hat{j}}\right) Y^{\hat{j}}
$$

This tells us that for a shear-free congruence $\left(\sigma_{\hat{i} \hat{j}}=0\right)$, if we lock the rotation $\vec{\Omega}$ of the tetrad to the vorticity $\vec{\omega}$ of the congruence, $\Omega_{\hat{i} \hat{j}}=\omega_{\hat{i} \hat{j}}$, the connecting vector's direction is fixed on the tetrad (and if in addition $\theta=0$, i.e., the congruence is rigid, the connecting vectors have constant components on the tetrad). A familiar example is the rigidly rotating frame in flat spacetime; in the non-relativistic limit, the vorticity of the congruence formed by the rigidly rotating observers is constant, and equals the angular velocity of the frame; in this case, by choosing $\vec{\Omega}=\vec{\omega}$, one is demanding that the spatial triads $\mathbf{e}_{\hat{i}}$ carried by the observers co-rotate with the angular velocity of the congruence; hence it is clear that the axes $\mathbf{e}_{\hat{i}}$ always point to the same neighboring observers. For relativistic rotation, the vorticity $\vec{\omega}$ is not constant and no longer equals the (constant) angular velocity of the rotating observers; but it is still the condition $\vec{\Omega}=\vec{\omega}$ that ensures that the tetrads are rigidly anchored to the observer congruence. Another example is the family of the so-called "static" observers in Kerr spacetime, which is very important in this context, because it is this construction which allows us to determine the rotation of the frame of the "distant stars" with respect to a local gyroscope, as we shall see in Sec. 3.3 .

\subsection{Geodesics. Inertial forces — "gravitoelectromagnetic fields"}

The spatial part of the geodesic equation for a test particle of 4 -velocity $U^{\alpha}, \nabla_{\mathbf{U}} U^{\alpha} \equiv D U^{\alpha} / d \tau=0$, reads, in the frame $e_{\hat{\alpha}}$ :

$$
\frac{d U^{\hat{i}}}{d \tau}+\Gamma_{\hat{0} \hat{0}}^{\hat{i}}\left(U^{\hat{0}}\right)^{2}+\left(\Gamma_{\hat{0} \hat{j}}^{\hat{i}}+\Gamma_{\hat{j} \hat{0}}^{\hat{i}}\right) U^{\hat{0}} U^{\hat{j}}+\Gamma_{\hat{j} \hat{k}}^{\hat{i}} U^{\hat{k}} U^{\hat{j}}=0
$$

Substituting (38), 39) and (49), we have

$$
\frac{\tilde{D} \vec{U}}{d \tau}=U^{\hat{0}}\left[U^{\hat{0}} \vec{G}+\vec{U} \times \vec{H}-\sigma^{\hat{i}}{ }_{j} U^{\hat{j}} \mathbf{e}_{\hat{i}}-\frac{1}{3} \theta \vec{U}\right] \equiv \vec{F}_{\mathrm{GEM}}
$$

where

$$
\frac{\tilde{D} U^{\hat{i}}}{d \tau}=\frac{d U^{\hat{i}}}{d \tau}+\Gamma_{\hat{j} \hat{k}}^{\hat{i}} U^{\hat{k}} U^{\hat{j}}
$$

Here $\vec{G}=-\vec{a}$ is the "gravitoelectric field", and $\vec{H}=\vec{\omega}+\vec{\Omega}$ is the "gravitomagnetic field". These designations are due to the analogy with the roles that the electric and magnetic fields play in the electromagnetic Lorentz force, which reads in the tetrad

$$
\left(\nabla_{\mathbf{U}} \mathbf{U}\right)^{\hat{i}}=\frac{q}{m}\left[U^{\hat{0}} E^{\hat{i}}+(\vec{U} \times \vec{B})^{\hat{i}}\right]
$$

with $\vec{E} \equiv \vec{E}(u)$ and $\vec{B} \equiv \vec{B}(u)$ denoting the electric and magnetic fields as measured by the observers $u^{\alpha}$. It is useful to write the GEM fields in a manifestly covariant form:

$$
\left(G^{u}\right)^{\alpha}=-\nabla_{\mathbf{u}} u^{\alpha} \equiv-u_{; \beta}^{\alpha} u^{\beta} ; \quad\left(H^{u}\right)^{\alpha}=\omega^{\alpha}+\Omega^{\alpha}
$$


The gravitomagnetic field $\left(H^{u}\right)^{\alpha}$ thus consists of two parts of different origins: the angular velocity $\Omega^{\alpha}$ of rotation of the spatial triads relative to Fermi-Walker transport (i.e., to local guiding gyroscopes), plus the vorticity $\omega^{\alpha}$ of the congruence of observers $u^{\alpha}$. If we lock the rotation of the triads to the vorticity of the congruence, $\Omega^{\alpha}=\omega^{\alpha}$, the gravitomagnetic field becomes simply twice the vorticity: $\left(H^{u}\right)^{\alpha}=2 \omega^{\alpha}$.

The last two terms of (52) have no electromagnetic counterpart; they consist of the shear/expansion tensor $K_{(\alpha \beta)}$, which is sometimes called the second fundamental form of the distribution of hyperplanes orthogonal to $\mathbf{u}$. If this distribution is integrable (that is, if there is no vorticity) then $K_{(\alpha \beta)}$ is just the extrinsic curvature of the time slices orthogonal to $\mathbf{u}$. These terms correspond to the time derivative of the spatial metric $\left(h^{u}\right)_{\alpha \beta}$, that locally measures the spatial distances between neighboring observers; this can be seen noting that $2 K_{(\alpha \beta)}=\left(h^{u}\right)^{\gamma}{ }_{\alpha}\left(h^{u}\right)^{\delta}{ }_{\beta} \mathcal{L}_{\mathbf{u}}\left(h^{u}\right)_{\gamma \delta}=u^{0} \partial_{0}\left(h^{u}\right)_{\alpha \beta}$, the last equality holding in a coordinate system where $\mathbf{u}$ is proportional to $\partial / \partial t$.

\subsubsection{The derivative operator $\tilde{D} / d \tau$ and inertial forces}

$\vec{F}_{\mathrm{GEM}}=\tilde{D} \vec{U} / d \tau$ describes the inertial accelerations (forces) associated to an arbitrary orthonormal frame; we shall now justify this statement, and the splitting of the connection made in Eq. (52). We start by noticing that $\tilde{D} \vec{U} / d \tau$ is a spatial vector which is the derivative of another spatial vector (the spatial velocity $U^{\langle\alpha\rangle}=\left(h^{u}\right)_{\beta}^{\alpha} U^{\beta}$, or $\vec{U}$ in the tetrad $\mathbf{e}_{\hat{\alpha}}$ ); mathematically, this is determined by a connection on the vector bundle of all spatial vectors. There is a Riemannian metric naturally defined on this vector bundle, the spatial metric $\left(h^{u}\right)_{\alpha \beta}$, and the most obvious connection preserving it is the orthogonal projection $\nabla^{\perp}$ of the ordinary spacetime covariant derivative,

$$
\nabla_{\alpha}^{\perp} X^{\beta} \equiv\left(h^{u}\right)_{\gamma}^{\beta} \nabla_{\alpha} X^{\gamma}
$$

which in terms of the tetrad components is written

$$
\nabla_{\hat{\alpha}}^{\perp} X^{\hat{i}}=\nabla_{\hat{\alpha}} X^{\hat{i}}=X_{, \hat{\alpha}}^{\hat{i}}+\Gamma_{\hat{\alpha} \hat{j}}^{\hat{i}} X^{\hat{j}}
$$

We shall call $\nabla^{\perp}$ the Fermi-Walker connection, since the parallel transport that it defines along the congruence is exactly the Fermi-Walker transport; this is because the spatially projected covariant derivative of a spatial vector $X^{\alpha}$ equals its Fermi-Walker derivative:

$$
\nabla_{\mathbf{u}}^{\perp} X^{\alpha}=\left(h^{u}\right)_{\beta}^{\alpha} \nabla_{\mathbf{u}} X^{\beta}=\nabla_{\mathbf{u}} X^{\alpha}-u^{\alpha} X_{\beta} \nabla_{\mathbf{u}} u^{\beta}
$$

Along any curve with tangent vector $\mathbf{U}$ we have

$$
\begin{aligned}
\frac{D^{\perp} X^{\hat{i}}}{d \tau} & \equiv \nabla_{\mathbf{U}}^{\perp} X^{\hat{i}}=\frac{d X^{\hat{i}}}{d \tau}+\Gamma_{\hat{0} \hat{j}}^{\hat{i}} U^{\hat{0}} X^{\hat{j}}+\Gamma_{\hat{j} \hat{k}}^{\hat{i}} U^{\hat{j}} X^{\hat{k}} \\
& =\frac{d X^{\hat{i}}}{d \tau}+\Omega^{\hat{i}}{ }_{\hat{j}} U^{\hat{0}} X^{\hat{j}}+\Gamma_{\hat{j} \hat{k}}^{\hat{i}} U^{\hat{j}} X^{\hat{k}}
\end{aligned}
$$

and so along the congruence,

$$
\nabla_{\mathbf{u}}^{\perp} X^{\hat{i}}=\dot{X}^{\hat{i}}+\Omega_{\hat{j}}^{\hat{i}} X^{\hat{j}}
$$


Notice that the Fermi-Walker connection preserves the spatial metric: if $\vec{X}$ and $\vec{Y}$ are spatial vector fields then we have

$$
\begin{aligned}
\frac{d}{d \tau}(\vec{X} \cdot \vec{Y}) & =\frac{d}{d \tau}\left(\delta_{\hat{i} \hat{j}} X^{\hat{i}} Y^{\hat{j}}\right)=\delta_{\hat{i} \hat{j}}\left(\frac{d X^{\hat{i}}}{d \tau} Y^{\hat{j}}+X^{\hat{i}} \frac{d Y^{\hat{j}}}{d \tau}\right)=\delta_{\hat{i} \hat{j}}\left(\frac{D^{\perp} X^{\hat{i}}}{d \tau} Y^{\hat{j}}+X^{\hat{i}} \frac{D^{\perp} Y^{\hat{j}}}{d \tau}\right) \\
& =\frac{D^{\perp} \vec{X}}{d \tau} \cdot \vec{Y}+\vec{X} \cdot \frac{D^{\perp} \vec{Y}}{d \tau},
\end{aligned}
$$

where we used $\Omega^{\hat{i}}{ }_{\hat{j}}=-\Omega_{\hat{i}}^{\hat{j}}$ and $\Gamma_{\hat{j} \hat{k}}^{\hat{i}}=-\Gamma_{\hat{j} \hat{i}}^{\hat{k}}$.

Eq. (59) yields the variation, along a curve of tangent $\mathbf{U}$, of a spatial vector $X^{\alpha}$, with respect to a triad of spatial axes undergoing Fermi-Walker transport along the congruence. But our goal is to measure "accelerations" (i.e, the variation of the spatial velocity $U^{\langle\alpha\rangle} \equiv\left(h^{u}\right)_{\beta}^{\alpha} U^{\beta}$ ) with respect to some chosen orthonormal frame, whose triad of spatial vectors $\mathbf{e}_{\hat{i}}$ rotate along the congruence (according to the Fermi-Walker connection, cf. Eq. (37)) with an angular velocity $\vec{\Omega}$ that one may arbitrarily specify. We need thus to define a connection for which the $\mathbf{e}_{\hat{i}}$ are constant along $\mathbf{u}$, whilst still equaling the projection $\left(\nabla^{\perp}\right)$ of the spacetime covariant derivative $\nabla$ along the directions orthogonal to $\mathbf{u}$ (so that it still corrects, via the coefficients $\Gamma_{\hat{j} \hat{k}}^{\hat{i}}$, for the variation ${ }^{11}$ of the $\mathbf{e}_{\hat{i}}$ in the directions orthogonal to the congruence, which are not related with inertial forces). This is achieved by the connection

$$
\tilde{\nabla}_{\alpha} X^{\beta}=\nabla_{\alpha}^{\perp} X^{\beta}+u_{\alpha}\left(h^{u}\right)_{\gamma}^{\beta} \Omega_{\delta}^{\gamma} X^{\delta}=\left(h^{u}\right)_{\gamma}^{\beta} \nabla_{\alpha} X^{\gamma}+u_{\alpha}\left(h^{u}\right)_{\gamma}^{\beta} \Omega_{\delta}^{\gamma} X^{\delta},
$$

or, in tetrad components,

$$
\tilde{\nabla}_{\hat{\alpha}} X^{\hat{i}}=\nabla_{\hat{\alpha}}^{\perp} X^{\hat{i}}-\delta_{\hat{\alpha}}^{\hat{0}} \Omega_{\hat{j}}^{\hat{i}} X^{\hat{j}}=X_{, \hat{\alpha}}^{\hat{i}}+\Gamma_{\hat{\alpha} \hat{j}}^{\hat{i}} X^{\hat{j}}-\delta_{\hat{\alpha}}^{0} \Omega^{\hat{i}}{ }_{\hat{j}} X^{\hat{j}},
$$

so that

$$
\frac{\tilde{D} X^{\hat{i}}}{d \tau}=\nabla_{\mathbf{U}}^{\perp} X^{\hat{i}}-\Omega_{\hat{j}}^{\hat{i}} U^{\hat{0}} X^{\hat{j}}=\frac{d X^{\hat{i}}}{d \tau}+\Gamma_{\hat{j} \hat{k}}^{\hat{i}} U^{\hat{j}} X^{\hat{k}} .
$$

Similarly to what was done for $\nabla^{\perp}$, it is easy to see that $\tilde{\nabla}$ preserves the spatial metric:

$$
\frac{d}{d \tau}(\vec{X} \cdot \vec{Y})=\frac{\tilde{D} \vec{X}}{d \tau} \cdot \vec{Y}+\vec{X} \cdot \frac{\tilde{D} \vec{Y}}{d \tau}
$$

Hence $\tilde{D} / d \tau$ is a covariant derivative along a curve, preserving the spatial metric $\left(h^{u}\right)_{\alpha \beta}$, for spatial vectors. The inertial forces $\left(F_{\mathrm{GEM}}^{\alpha}\right)$ of a given frame are given by the derivative $\tilde{D} / d \tau$ acting on the spatial velocity $U^{\langle\alpha\rangle}$ of a particle undergoing geodesic motion. In covariant form, we have

$$
F_{\mathrm{GEM}}^{\alpha} \equiv \frac{\tilde{D} U^{\langle\alpha\rangle}}{d \tau}=\frac{D^{\perp} U^{\langle\alpha\rangle}}{d \tau}+\gamma \epsilon_{\beta \gamma \delta}^{\alpha} u^{\delta} U^{\beta} \Omega^{\gamma}=-\gamma \frac{D^{\perp} u^{\alpha}}{d \tau}+\gamma \epsilon_{\beta \gamma \delta}^{\alpha} u^{\delta} U^{\beta} \Omega^{\gamma}
$$

where $\gamma \equiv-U_{\alpha} u^{\alpha}$. In the last equality, we decomposed $U^{\alpha}$ into its projections parallel and orthogonal to the congruence, $U^{\alpha}=\gamma u^{\alpha}+U^{\langle\alpha\rangle}$, and used the geodesic equation, $D U^{\alpha} / d \tau=0$, to note that $D^{\perp} U^{\langle\alpha\rangle} / d \tau=-\gamma D^{\perp} u^{\alpha} / d \tau$. Eq. 64 manifests that $F_{\text {GEM }}^{\alpha}$ consists of two terms of distinct origin: the first term which depends only on the variation of the observer velocity $u^{\alpha}$ along

\footnotetext{
${ }^{11}$ e.g. the trivial variation from point to point of the triads associated with a non-rectangular coordinate system in flat spacetime. These do not encode inertial forces, nor do they necessarily vanish in an inertial frame.
} 
the test particle's worldline, and the second term which is independent of the observer congruence, and arises from the transport law for the spatial triads along $u^{\alpha}$. These two contributions are illustrated, for simple examples in flat spacetime, in Appendix A.

Using $D u^{\alpha} / d \tau=u^{\alpha ; \beta} U_{\beta}$, and decomposing $u_{\alpha ; \beta}$ in the congruence kinematics, cf. Eqs. (44), (46), (47),

$$
u_{\alpha ; \beta}=-a(u)_{\alpha} u_{\beta}-\epsilon_{\alpha \beta \gamma \delta} \omega^{\gamma} u^{\delta}+K_{(\alpha \beta)},
$$

we get, substituting into $64{ }^{12}$

$$
F_{\mathrm{GEM}}^{\alpha}=\gamma\left[\gamma G^{\alpha}+\epsilon_{\beta \gamma \delta}^{\alpha} u^{\delta} U^{\beta}\left(\omega^{\gamma}+\Omega^{\gamma}\right)-K^{(\alpha \beta)} U_{\beta}\right]
$$

which is Eq. (52) in covariant form.

The derivative (53) has a geometrical interpretation that is more familiar when $\Omega^{\alpha}=\omega^{\alpha}$ and the restriction of $\tilde{\nabla}$ to the spatial directions can be interpreted as the Levi-Civita connection of some 3-D Riemannian manifold. However, as we will see, this happens only in special cases. The quotient of the spacetime by the congruence is diffeomorphic to any time slice $\Sigma$, locally given by $t=f\left(x^{i}\right)$ in a coordinate system where $\mathbf{u}$ is proportional to $\partial / \partial t$. The restriction to $\Sigma$ of the spatial projection of the 4-D metric, $\left.\left.\left(h^{u}\right)_{\alpha \beta}\right|_{\Sigma} \equiv h_{\alpha \beta}\right|_{\Sigma}$, yields the spatial distances between neighboring observers along the slice; choosing it to be the Riemannian metric on $\Sigma$ leads, in general, to a slice dependent metric, $\left.h_{\alpha \beta}\right|_{\Sigma} \equiv h_{\alpha \beta}\left(f\left(x^{i}\right), x^{i}\right)$. Let $\mathbf{E}_{\hat{i}}=\mathbf{e}_{\hat{i}}+A_{\hat{i}} \mathbf{e}_{\hat{0}}$ be tangent to $\Sigma$; they form (by construction) an orthonormal basis with respect to $\left.h_{\alpha \beta}\right|_{\Sigma}$. Let $\Gamma(\Sigma)_{\hat{i} \hat{j}}^{\hat{k}}$ be the Levi-Civita connection coefficients of $\left.h_{\alpha \beta}\right|_{\Sigma}$ in this basis. Using the vanishing of the torsion, $\left[\mathbf{E}_{\hat{j}}, \mathbf{E}_{\hat{k}}\right]=2 \Gamma(\Sigma)_{[\hat{j} \hat{k}]}^{\hat{i}} \mathbf{e}_{\hat{i}}$, and anti-symmetry in $\hat{i}, \hat{k}$, one can show after some algebra that

$$
\Gamma(\Sigma)_{\hat{j} \hat{k}}^{\hat{i}}=\Gamma_{\hat{j} \hat{k}}^{\hat{i}}+A_{\hat{k}} K_{(\hat{i} \hat{j})}-A_{\hat{i}} K_{(\hat{j} \hat{k})}
$$

(for an equivalent expression in terms of a coordinate basis, see Eq. (10.10) of [27]). This tells us that the coefficients $\Gamma_{\hat{j} \hat{k}}^{\hat{i}}$ match $\Gamma(\Sigma)_{\hat{j} \hat{k}}^{\hat{i}}$ in two notable cases: (i) when $\omega^{\alpha}=0$ and/or (ii) $K_{(\alpha \beta)}=0$. In case (i) the congruence is hypersurface orthogonal; let $\Sigma_{t}$ be one such particular hypersurface; it follows that $A_{i}=0 \Rightarrow \Gamma(\Sigma)_{\hat{j} k}^{\hat{i}}=\Gamma_{\hat{j} \hat{k}}^{\hat{i}}$. This is a natural result if we note that $\left.h_{\alpha \beta}\right|_{\Sigma}$ is in this case the induced metric on $\Sigma_{t}$, whose Levi-Civita connection is well known (e.g. [12], Lemma 10.2.1) to be the projection of the spacetime connection $\nabla$ on $\Sigma_{t}$. In case (ii) the congruence is not hypersurface orthogonal; but on the other hand it is rigid (i.e., the distance between neighboring observers is constant along $\mathbf{u}$ ), and so $\left.h_{\alpha \beta}\right|_{\Sigma}=h_{\alpha \beta}$ is independent of the time slice (see e.g. [19] p. 221; indeed in this case one can identify the quotient with a Riemannian manifold $(\Sigma, \boldsymbol{h})$ ). Therefore at each point of the quotient one can choose a slice which is orthogonal to the congruence at that point, and an argument similar to the above applies.

Let us now see the geometrical meaning of $\vec{F}_{\mathrm{GEM}}=\tilde{D} \vec{U} / d \tau$ in these two cases. Consider, in case (ii), the 3-D curve obtained by projecting the particle's worldline $z^{\alpha}(\tau)$ on the (time independent) space manifold $\Sigma$. Let it still be parametrized by $\tau$; then $\vec{U}$ is its tangent vector, and $\tilde{D} \vec{U} / d \tau$ is just

\footnotetext{
${ }^{12}$ This corresponds to a generalized version, for arbitrary orthonormal frames, of Eqs. (6.13) or (6.18) of [27, which in their scheme would follow from a "derivative" of the type (5.3), but allowing for an arbitrary $\Omega^{\alpha}$, rather than the two choices $\Omega^{\alpha}=0$ and $\Omega^{\alpha}=\omega^{\alpha}$ ("fw" and "cfw" in their notation, respectively), cf. Eq. (2.16). On the other hand, their Lie transport option ("lie") in (5.3), which does not preserve orthonormality of the axes, is not encompassed in our derivative (64).
} 
the usual covariant derivative, with respect the spatial metric, of $\vec{U}$ - that is, the 3-D acceleration of this curve. Note that expression (53) is indeed identical to the usual definition of 3-D acceleration for curved spaces (or non-rectangular coordinate systems in Euclidean space), e.g. Eq. (6.9) of [113]. It is easy to see that this corresponds to the usual notion of inertial force from classical mechanics. Take a familiar example, a rigidly rotating frame in flat spacetime (as discussed in Appendix A]); we are familiar with the inertial forces arising in such frame, from e.g. a merry-go-round. They are in this case a gravitoelectric field $\vec{G}=\vec{\omega} \times(\vec{r} \times \vec{\omega})$, due to the acceleration of the rigidly rotating observers, that causes a centrifugal force, plus a gravitomagnetic field $\vec{H}=2 \vec{\omega}$, half of it originating from the observers' vorticity $\vec{\omega}$, and the other half from the angular velocity of rotation $\vec{\Omega}=\vec{\omega}$ (relative to Fermi-Walker transport) of the spatial triads they carry. $\vec{H}$ causes the Coriolis (or gravitomagnetic) force $\vec{U} \times \vec{H}=2 \vec{U} \times \vec{\omega}$. These centrifugal and Coriolis forces become, in the non-relativistic limit (so that the vorticity $\vec{\omega}$ equals the angular velocity of the rotating frame) the well known expressions from non-relativistic mechanics e.g. Eq. (4.91) of [114]; the derivative operator $\tilde{D} / d \tau$ becomes the one in Eq. (4.86) therein, and Eq. 63 becomes Eq. (4.82) therein, identifying $\nabla_{\mathbf{U}}^{\perp} X^{\hat{i}}$ and $\tilde{D} X^{\hat{i}} / d \tau$, respectively, with their "time derivatives observed in the space (i.e. non-rotating) and rotating frames".

Let us turn now to the sub-case $\omega^{\alpha}=0$. If $K_{(\alpha \beta)}=0$ (static congruence), $\vec{F}_{\text {GEM }}$ is just the 3-D acceleration of the projection of the particle's worldline on the time independent hypersurfaces $\Sigma$. If $K_{(\alpha \beta)} \neq 0$, the geometric interpretation of $\vec{F}_{\mathrm{GEM}}$ is a bit trickier because $\Sigma_{t}$ changes with time. In this case, we can make a point-wise interpretation: at each point, it is the 3-D acceleration of the projected curve on that particular $\Sigma_{t}$ at that point.

In the more general case $\omega^{\alpha} \neq 0$ and $K_{(\alpha \beta)} \neq 0$, and/or if $\omega^{\alpha} \neq \Omega^{\alpha}$ (case of the so-called "locally non-rotating frames", see below), it is not possible to interpret (53) as the acceleration of a projected curve in some space manifold; but it still yields what one would call the inertial forces of the given frame, which is exemplified in the case of flat spacetime in Appendix A.

Usefulness of the general equation. - An equation like (52), yielding the inertial forces in an arbitrary orthonormal frame, in particular allowing for an arbitrary rotation $\Omega^{\alpha}$ of the spatial triads along $u^{\alpha}$, independent of $\omega^{\alpha}$, is of interest in many applications. Although the congruence adapted frame, $\vec{\Omega}=\vec{\omega}=\vec{H} / 2$, might seem the most natural frame associated to a given family of observers, other frames are used in the literature, and the associated gravitomagnetic effects (encompassed in our general definition of $\vec{H}$, Eq. 55) discussed therein. That includes the case of the reference frames sometimes employed in the context of black hole physics and astrophysics [26, 102, 103, 104, 105, 52]: the tetrads carried by hypersurface orthogonal observers, whose spatial axis are taken to be fixed to the background symmetries; for instance, in the Kerr spacetime, the congruence are the zero angular momentum observers (ZAMOS), and the spatial triads are fixed to the Boyer-Lindquist spatial coordinate basis vectors. This tetrad field has been dubbed in some literature "locally non-rotating frames" $(\mathrm{LNR}){ }^{13}$ [103, 102, 104, 26] or "proper reference frames of the fiducial observers" [25]. It is regarded as important for black hole physics because it is a reference frame that is defined everywhere (unlike for instance the star fixed static observers, see Sec. 3.3 below, that do not exist past the ergosphere). Eq. (52) allows the description of the inertial forces in these frames, where $\vec{\omega}=0$, and $\vec{H}=\vec{\Omega}$; that is, all the gravitomagnetic accelerations

\footnotetext{
${ }^{13}$ Somewhat erroneously, as the tetrads do rotate with respect to the local compass of inertia, since they are not Fermi-Walker transported in general [52, 104, 105.
} 
come from ${ }^{14} \vec{\Omega}$ (for explicit expressions of $\vec{\Omega}$, see e.g. Eq. (33.24) of [52], or Eq. (75) of [104]). Frames corresponding to a congruence with vorticity, but where the spatial triads are chosen to be Fermi-Walker transported, $\vec{\Omega}=0$, have also been considered; in such frames $\vec{H}=\vec{\omega}$ (dubbed the "Fermi-Walker gravitomagnetic field" [27, 28]).

Finally, it is worth noting that the GEM "Lorentz" forces from the more popular linearized theory, e.g. [7, 8, 11, 9, 14, or post-Newtonian approaches, e.g. [67, 62, 61], are special cases of Eq. (52) (e.g. linearizing it, one obtains Eq. (2.5) of [51]; further specializing to stationary fields, one obtains e.g. (6.1.26) of [7]).

\subsubsection{Stationary fields — "quasi-Maxwell" formalism}

If one considers a stationary spacetime, and a frame where it is explicitly time-independent (i.e., a congruence of observers $u^{\alpha}$ tangent to a time-like Killing vector field, which necessarily means that the congruence is rigid [99]), the last two terms of Eq. (52) vanish and the geodesic equation becomes formally similar to the Lorentz force (54):

$$
\frac{\tilde{D} \vec{U}}{d \tau}=U^{\hat{0}}\left(U^{\hat{0}} \vec{G}+\vec{U} \times \vec{H}\right) .
$$

The line element of a stationary spacetime is generically described by:

$$
d s^{2}=-e^{2 \Phi}\left(d t-\mathcal{A}_{i} d x^{i}\right)^{2}+h_{i j} d x^{i} d x^{j}
$$

with $\Phi, \overrightarrow{\mathcal{A}}, h_{i j}$ time-independent. Here $h_{i j}=\left(h^{u}\right)_{i j}$ is a Riemannian metric, not flat in general, that measures the time-constant distance between stationary observers, as measured by the Einstein light signaling procedure [18]. As discussed above, Eq. 68 is the acceleration of the 3-D curves obtained by projecting test-particle's geodesics in the 3 -D manifold of metric $h_{i j}$. The GEM fields measured by the static observers (i.e. the observers of zero 3-velocity in the coordinate system of (69)) are related with the metric potentials by [20]:

$$
\vec{G}=-\tilde{\nabla} \Phi ; \quad \vec{H}=e^{\Phi} \tilde{\nabla} \times \overrightarrow{\mathcal{A}},
$$

with $\tilde{\nabla}$ denoting the covariant differentiation operator with respect to the spatial metric $h_{i j}$. The formulation (70) of GEM fields applying to stationary spacetimes is the most usual one; it was introduced in [18], and further worked out in e.g. [21, 20, 25, 23, 19], and is sometimes called the "quasi-Maxwell formalism".

\subsection{Gyroscope precession}

One of the main results of this approach is that, within this formalism, the equation describing the evolution of the spin-vector of a gyroscope in a gravitational field takes a form exactly analogous to the precession of a magnetic dipole under the action of a magnetic field when expressed in a local orthonormal tetrad comoving with the test particle.

As we have seen in Sec. 2.3. if the Mathisson-Pirani condition holds, the spin vector of a torque-free gyroscope is Fermi-Walker transported, cf. Eq. (27). Let $U^{\alpha}$ be the 4 -velocity of the

\footnotetext{
${ }^{14}$ In [26] the term involving $\Omega^{\alpha}$ in Eq. 66 above is cast not as part of a gravitomagnetic, but of a "Coriolis" acceleration (" $a_{\mathrm{C}}^{\alpha}$ "). Therein, what is cast as "gravitomagnetic" (" $a_{\mathrm{d}}^{\alpha}$ "), are the terms involving $K_{(\alpha \beta)}$ and $\omega^{\alpha}$.
} 
gyroscope; in a comoving orthonormal tetrad $e_{\hat{\alpha}}, U^{\hat{\alpha}}=\delta_{\hat{0}}^{\hat{\alpha}}$ and $S^{\hat{0}}=0$; therefore, Eq. 27] reduces in such frame to:

$$
\frac{D S^{\hat{i}}}{d \tau}=0 \Leftrightarrow \frac{d S^{\hat{i}}}{d \tau}=-\Gamma_{\hat{0} \hat{k}}^{\hat{i}} S^{\hat{k}}=(\vec{S} \times \vec{\Omega})^{\hat{i}}
$$

This is the natural result. If we choose a Fermi-Walker transported frame, obtained by setting in Eq. (37) $\Omega^{\alpha}=0, u^{\alpha}=U^{\alpha}$ (mathematically this is defined as a frame with no "absolute" spatial rotation), then gyroscopes, which are understood as objects that "oppose" to changes in direction (and interpreted as determining the local "compass of inertia" [7]), have their axes fixed with respect to such frame: $d \vec{S} / d \tau=0$. Otherwise gyroscopes are seen to "precesses" with an angular velocity $-\vec{\Omega}$, that is in fact just minus the angular velocity of rotation of the chosen frame relative to a Fermi-Walker transported frame. Now, for a congruence adapted frame, $\vec{\Omega}=\vec{\omega}$, Eq. 71 becomes:

$$
\frac{d \vec{S}}{d \tau}=\frac{1}{2} \vec{S} \times \vec{H}
$$

Thus, the "precession" of a gyroscope is given, in terms of the gravitomagnetic field $\vec{H}$, by an expression identical (up to a factor of 2) to the precession of a magnetic dipole under the action of a magnetic field $\vec{B}$, cf. Eq. 31):

$$
\frac{D \vec{S}}{d \tau}=\vec{\mu} \times \vec{B} .
$$

This holds for arbitrary fields, hence in this case the one to one correspondence with electromagnetism is more general than the one for the geodesics described above (between Eqs. (68) and (54)), which required the fields to be stationary and the observers to be stationary (i.e., their worldlines be tangent to a Killing vector field); herein by contrast the only conditions are the observer to be comoving with the gyroscope, and using an orthonormal tetrad. Also, the earlier result obtained for weak fields in [51] (that the analogy holds even if the fields are time dependent) is just a special case of this principle.

However important differences should be noted: whereas in the electromagnetic case it is the same field $\vec{B}$ that is at the origin of both the magnetic force $q(\vec{U} \times \vec{B})$ in Eq. 54 and the torque $\vec{\mu} \times \vec{B}$ on the magnetic dipole, in the case of the gravitomagnetic force $\vec{U} \times \vec{H}$ it has, in the general formulation, a different origin from gyroscope "precession", since the former arises not only from the rotation $\vec{\Omega}$ of the frame relative to a local Fermi-Walker transported tetrad, but also from the vorticity $\vec{\omega}$ of the congruence. In this sense, one can say that the Lense-Thirring effect detected in the LAGEOS satellite data [58] (and presently under experimental scrutiny by the ongoing LARES mission [60]), measuring $\vec{H}$ from test particle's deflection, is of a different mathematical origin from the one which was under scrutiny by the Gravity Probe B mission [59], measuring $\vec{\Omega}$ from gyroscope precession, the two being made to match by measuring both effects relative to the "frame of the distant stars" (discussed below), for which $\vec{\Omega}=\vec{\omega}=\vec{H} / 2$. This type of frame (i.e. congruence adapted) is the most usual in the literature; in this case the fields causing the gravitomagnetic force and the precession of a gyroscope differ only by a relative factor of 2 . But it is important to not overlook their distinct origin, as in the literature GEM fields of frames which are not congruence adapted are considered as well; for instance the "Fermi-Walker gravitomagnetic field" defined in [27], which is the $\vec{H}$ of a frame corresponding to a congruence with vorticity, but where the spatial triads are chosen to be Fermi-Walker transported: $\vec{\Omega}=0$. Thus there is a non-vanishing $\vec{H}=\vec{\omega}$ in this frame, whereas at the same time gyroscopes do not precess relative to it. 
Another obvious difference between Eqs. (73) and (72) is the presence of a covariant derivative in the former, whereas in the latter we have a simple derivative, signaling that $\vec{B}$ is a physical field, and $\vec{H}$ a mere artifact of the reference frame (which can be anything, depending on the frame one chooses), that can always be made to vanish (in the congruence adapted case, $\vec{H}=2 \vec{\omega}$, by choosing a vorticity-free observer congruence).

Frame dragging. - The fact that $\vec{H}$ is a reference frame artifact does not mean it is necessarily meaningless; indeed it has no local physical significance, but it can tell us about frame dragging, which is a non-local physical effect. That is the case when one chooses the so-called "frame of the distant stars", a notion that applies to asymptotically flat spacetimes. In stationary spacetimes, such frame is setup as follows: consider a rigid congruence of stationary observers such that at infinity it coincides with the asymptotic inertial rest frame of the source - the axes of the latter define the directions fixed relative to the distant stars. These observers are interpreted as being "at rest" with respect to the distant stars (and also at rest with respect to the asymptotic inertial frame of the source); since the congruence is rigid, it may be thought as a grid of points rigidly fixed to the distant stars. For this reason we dub them "static observers' 15 , This congruence fixes the time axis of the local tetrads of the frame. Now if we demand the rotation $\vec{\Omega}$ of the spatial triads (relative to Fermi-Walker transport) to equal the vorticity $\vec{\omega}$ of the congruence, we see from Eq. (51) that the spatial components of the connecting vectors between different observers are constant in the tetrad:

$$
\dot{Y}^{\hat{i}}=0 ;
$$

in other words, each local spatial triad $e_{\hat{i}}$ is locked to this grid, and therefore has directions fixed to the distant stars. Hence, despite having no local meaning, the gravitomagnetic field $\vec{H}=2 \vec{\Omega}=2 \vec{\omega}$ describes in this case a consequence of the frame dragging effect: the fact that a torque free gyroscope at finite distance from a rotating source precesses with respect to an inertial frame at infinity. This is a physical effect, that clearly distinguishes, for instance, the Kerr from the Schwarzschild spacetimes, but is non-local (i.e., it cannot be detected in any local measurement; only by locking to the distant stars by means of telescopes). It should be noted, however, that the relative precession of two neighboring (comoving) systems of gyroscopes is locally measurable, and encoded in the curvature tensor (more precisely, in the gravitomagnetic tidal tensor $\mathbb{H}_{\alpha \beta}$, as discussed in Sec. 2.3).

\subsection{Field equations}

The Einstein field equations and the algebraic Bianchi identity, Eqs. (14), can be generically written in this exact GEM formalism - i.e., in terms of $\vec{G}, \vec{\Omega}, \vec{\omega}$ and $K_{(\alpha \beta)}$. These equations will be compared in this section with the analogous electromagnetic situation: Maxwell's equations in an arbitrarily accelerated, rotating and shearing frame. The latter will be of use also in Sec. 6. As a special case, we will also consider stationary spacetimes (and rigid, congruence adapted frames therein), where we recover the quasi-Maxwell formalism of e.g. [20, 24, 18, 22, 23, 19]. In this case,

\footnotetext{
${ }^{15}$ In the case of Kerr spacetime, these are the observers whose worldlines are tangent to the temporal Killing vector field $\xi=\partial / \partial t$, i.e., the observers of zero 3-velocity in Boyer-Lindquist coordinates. This agrees with the convention in [28, 98, 52, 102]. We note however that the denomination "static observers" is employed in some literature (e.g. 87, 88]) with a different meaning, where it designates hypersurface orthogonal time-like Killing vector fields (which are rigid, vorticity-free congruences, existing only in static spacetimes).
} 
the similarity with the electromagnetic analogue - Maxwell's equations in a rigid, but arbitrarily accelerated and rotating frame - becomes closer, as we shall see.

Before proceeding, let us write the following relations which will be useful. Let $A^{\alpha}$ be a spatial vector; in the tetrad we have:

$$
\begin{aligned}
\tilde{\nabla}_{\hat{i}} A^{\hat{j}} & =A^{\hat{j}}{ }_{, \hat{i}}+\Gamma_{\hat{k} \hat{i}}^{\hat{j}} A^{\hat{k}} ; \\
\nabla_{\mathbf{u}} A^{\hat{i}} & =\dot{A}^{\hat{i}}+\Gamma_{\hat{0} \hat{j}}^{\hat{i}} A^{\hat{j}}=\dot{A}^{\hat{i}}+\Omega^{\hat{i}} A^{\hat{j}} ; \\
A_{; \beta}^{\beta} & =\left(A_{, \hat{i}}^{\hat{i}}+A^{\hat{i}} \Gamma_{\hat{j} \hat{i}}^{\hat{j}}\right)+A^{\hat{i}} \Gamma_{\hat{0} \hat{i}}^{\hat{0}}=\tilde{\nabla} \cdot \vec{A}+\vec{A} \cdot \vec{a},
\end{aligned}
$$

where we used (62) and the connection coefficients (38)- $(39)$, and the dot denotes the ordinary derivative along the observer worldline, $\dot{A}^{\hat{\alpha}} \equiv A_{, \hat{\beta}}^{\hat{\alpha}} u^{\hat{\beta}}$. $\tilde{\nabla}$ is the connection defined in Eqs. 61)(62); since in expressions $(74)$ and $(76)$ the derivatives are along the spatial directions, one could as well have used the Fermi-Walker connection $\nabla^{\perp}$, Eqs. (56)-(57), they are the same along these directions.

\subsubsection{Maxwell equations for the electromagnetic fields measured by an arbitrary con- gruence of observers}

Using decomposition (1), we write Maxwell's Eqs. (6) in terms of the electric and magnetic fields $\left(E^{u}\right)^{\alpha}=F_{\beta}^{\alpha} u^{\beta}$ and $\left(B^{u}\right)^{\alpha}=\star F_{\beta}^{\alpha} u^{\beta}$ measured by the congruence of observers of 4 -velocity $u^{\alpha}$. All the fields below are measured with respect to this congruence, so we may drop the superscripts $u$ : $\left(E^{u}\right)^{\alpha} \equiv E^{\alpha},\left(B^{u}\right)^{\alpha} \equiv B^{\alpha}$. The time projection of Eq. (6a) with respect to $u^{\alpha}$ (see point 2 of Sec. 1.1) reads:

$$
E_{; \beta}^{\beta}=4 \pi \rho_{c}+E^{\alpha} a_{\alpha}+2 \omega_{\alpha} B^{\alpha} .
$$

Using (76), we have in the tetrad:

$$
\tilde{\nabla} \cdot \vec{E}=4 \pi \rho_{c}+2 \vec{\omega} \cdot \vec{B}
$$

Analogously, for the time projection of $(6 \mathrm{~b})$ we get

$$
B_{; \beta}^{\beta}=B^{\alpha} a_{\alpha}-2 \omega^{\mu} E_{\mu}
$$

which in the tetrad becomes

$$
\tilde{\nabla} \cdot \vec{B}=-2 \vec{\omega} \cdot \vec{E}
$$

The space projection of Eq. (6a) reads:

$$
\epsilon^{\alpha \gamma \beta} B_{\beta ; \gamma}=\nabla_{\mathbf{u}}^{\perp} E^{\alpha}-K^{(\alpha \beta)} E_{\beta}+\theta E^{\alpha}-\epsilon_{\beta \gamma}^{\alpha} \omega^{\beta} E^{\gamma}+\epsilon_{\beta \gamma}^{\alpha} B^{\beta} a^{\gamma}+4 \pi j^{\langle\alpha\rangle},
$$

where the index notation $\langle\mu\rangle$ stands for the spatially projected part of a vector, $V_{\langle\mu\rangle} \equiv h_{\mu}{ }^{\nu} V_{\nu}$, and $\epsilon^{\mu \beta \sigma} \equiv \epsilon^{\mu \beta \sigma \alpha} u_{\alpha}$. The tetrad components of 81 in the frame defined in Sec. 3.1 read:

$$
(\tilde{\nabla} \times \vec{B})^{\hat{i}}=\nabla_{\mathbf{u}} E^{\hat{i}}-K^{(\hat{i} \hat{j})} E_{\hat{j}}+\theta E^{\hat{i}}-(\vec{\omega} \times \vec{E})^{\hat{i}}+(\vec{G} \times \vec{B})^{\hat{i}}+4 \pi j^{\hat{i}} .
$$

Using (75), this becomes

$$
(\tilde{\nabla} \times \vec{B})^{\hat{i}}=\dot{E}^{\hat{i}}+(\vec{G} \times \vec{B})^{\hat{i}}+[(\vec{\Omega}-\vec{\omega}) \times \vec{E}]^{\hat{i}}-K^{(\hat{i} \hat{j})} E_{\hat{j}}+\theta E^{\hat{i}}+4 \pi j^{\hat{i}}
$$


The space projection of $(6 \mathrm{~b})$ is

$$
\epsilon^{\alpha \gamma \beta} E_{\beta ; \gamma}=-\nabla_{\mathbf{u}}^{\perp} B^{\alpha}+K^{(\alpha \beta)} B_{\beta}-\theta B^{\alpha}+\epsilon_{\beta \gamma}^{\alpha} \omega^{\beta} B^{\gamma}+\epsilon^{\alpha \mu \sigma} E_{\mu} a_{\sigma}
$$

which, analogously, reads in the tetrad,

$$
(\tilde{\nabla} \times \vec{E})^{\hat{i}}=-\dot{B}^{\hat{i}}+(\vec{G} \times \vec{E})^{\hat{i}}+[(\vec{\omega}-\vec{\Omega}) \times \vec{B}]^{\hat{i}}+K^{(\hat{i} \hat{j})} B_{\hat{j}}-\theta B^{\hat{i}} .
$$

In the congruence adapted frame $(\vec{\omega}=\vec{\Omega}=\vec{H} / 2)$, Eqs. 78, , 80, 83 and 85 above become,

$$
\begin{aligned}
\tilde{\nabla} \cdot \vec{E} & =4 \pi \rho_{c}+\vec{H} \cdot \vec{B} \\
\tilde{\nabla} \times \vec{B} & =\dot{\vec{E}}+\vec{G} \times \vec{B}+4 \pi \vec{j}-K^{(\hat{i} \hat{j})} E_{\hat{j}} \vec{e}_{\hat{i}}+\theta \vec{E} ; \\
\tilde{\nabla} \cdot \vec{B} & =-\vec{H} \cdot \vec{E} \\
\tilde{\nabla} \times \vec{E} & =-\dot{\vec{B}}+\vec{G} \times \vec{E}+K^{(\hat{i} \hat{j})} B_{\hat{j}} \vec{e}_{\hat{i}}-\theta \vec{B}
\end{aligned}
$$

In the special case of a rigid frame $\left(K^{(\hat{i} \hat{j})}=\theta=0\right)$ and time-independent fields $(\dot{\vec{E}}=\dot{\vec{B}}=0)$, this yields Eqs. (2.4a)-(3.8a) of Table 2 .

\subsubsection{Einstein equations}

We start by computing the tetrad components of the Riemann tensor in the frame of Sec. 3.1 ;

$$
\begin{aligned}
R_{\hat{0} \hat{i} \hat{j} \hat{j}} & =-\tilde{\nabla}_{\hat{i}} G_{\hat{j}}+G_{\hat{i}} G_{\hat{j}}-\dot{K}_{\hat{j} \hat{i}}+K_{\hat{l i}} \Omega_{\hat{j}_{\hat{j}}^{\hat{l}}}+\Omega_{{ }_{i}^{\hat{l}}} K_{\hat{j} \hat{l}}-K_{\hat{i}}^{\hat{l}} K_{\hat{j} \hat{l}} ; \\
R_{\hat{0} \hat{i} \hat{j} \hat{k}} & =\tilde{\nabla}_{\hat{k}} K_{\hat{i} \hat{j}}-\tilde{\nabla}_{\hat{j}} K_{\hat{i} \hat{k}}+2 G_{\hat{i}} \omega_{\hat{j} \hat{k}} \\
R_{\hat{i} \hat{j} \hat{k} \hat{l}} & =\tilde{R}_{\hat{i} \hat{j} \hat{k} \hat{l}}-K_{\hat{l} \hat{l}} K_{\hat{k} \hat{j}}+K_{\hat{l} \hat{j}} K_{\hat{k} \hat{i}}+2 \omega_{\hat{i} \hat{j}} \Omega_{\hat{k} \hat{l}} .
\end{aligned}
$$

In the expressions above we kept $\vec{\Omega}$ independent of $\vec{\omega}$, so that they apply to an arbitrary orthonormal tetrad field. Here

$$
\tilde{R}_{\hat{j} \hat{k} \hat{l}}^{\hat{i}} \equiv \Gamma_{\hat{l} \hat{j}, \hat{k}}^{\hat{i}}-\Gamma_{\hat{k} \hat{j}, \hat{l}}^{\hat{i}}+\Gamma_{\hat{k} \hat{m}}^{\hat{i}} \Gamma_{\hat{l} \hat{j}}^{\hat{m}}-\Gamma_{\hat{l} \hat{m}}^{\hat{i}} \Gamma_{\hat{k} \hat{j}}^{\hat{m}}-C_{\hat{k} \hat{l}}^{\hat{m}} \Gamma_{\hat{m} \hat{j}}^{\hat{i}}
$$

(where $C_{\hat{k} \hat{l}}^{\hat{m}}=\Gamma_{\hat{k} \hat{l}}^{\hat{m}}-\Gamma_{\hat{l} \hat{k}}^{\hat{m}}$ ) is the restriction to the spatial directions of the curvature of the connection $\tilde{\nabla}$, given by

$$
\tilde{R}(\vec{X}, \vec{Y}) \vec{Z}=\tilde{\nabla}_{\vec{X}} \tilde{\nabla}_{\vec{Y}} \vec{Z}-\tilde{\nabla}_{\vec{Y}} \tilde{\nabla}_{\vec{X}} \vec{Z}-\tilde{\nabla}_{[\vec{X}, \vec{Y}]} \vec{Z}
$$

for any spatial vector fields $\vec{X}, \vec{Y}, \vec{Z}$. It is related by

$$
\tilde{R}_{\hat{i} \hat{j} \hat{k} \hat{l}}=R_{\hat{i} \hat{j} \hat{k} \hat{l}}^{\perp}-2 \omega_{\hat{i} \hat{j}} \Omega_{\hat{k} \hat{l}}
$$

to the curvature tensor of the distribution of hyperplanes orthogonal to the congruence, $R_{\hat{i} \hat{j} \hat{k} \hat{k}}^{\perp}$, that is, the restriction to the spatial directions of the curvature of the Fermi-Walker connection $\nabla^{\perp}$ on the vector bundle of spatial vectors, given by a definition similar to 94 , only replacing $\tilde{\nabla} \rightarrow \nabla^{\perp}$.

In the special cases (i)-(ii) discussed in Sec. 3.2.1 - congruence adapted frames $\vec{\Omega}=\vec{\omega}$, and (i) $\omega^{\alpha}=0$ or (ii) $K_{(\alpha \beta)}=0$ - where the spatial restriction of $\tilde{\nabla}$ is the Levi-Civita connection of the spatial metric $\left(h^{u}\right)_{\alpha \beta}, \tilde{R}_{\hat{i} \hat{j} \hat{k} \hat{l}}$ has a simple interpretation, it is the curvature tensor of such metric. In case (i), the observers are hypersurface orthogonal (i.e., the distribution is integrable), 
and $R_{\hat{i} \hat{j} \hat{k} \hat{l}}^{\perp}=\tilde{R}_{\hat{i} \hat{j} \hat{k} \hat{l}}$ is the curvature of the hypersurfaces. In case (ii) $\tilde{R}_{\hat{i} \hat{j} \hat{k} \hat{l}} \neq R_{\hat{i} \hat{j} \hat{k} \hat{l}}^{\perp}$, and it is $\tilde{R}_{\hat{i} \hat{j} \hat{k} \hat{l}}$ (not $R_{\hat{i} \hat{j} \hat{k} \hat{l}}^{\perp}$ ) that yields the curvature of the space manifold $(\Sigma, \mathbf{h})$. In the general case however $\tilde{R}_{\hat{i} \hat{j} \hat{k} \hat{l}}$ cannot be identified with the Levi-Civita connection of some 3D sub-manifold16.

We shall now compute the tetrad components of the Ricci tensor, but specializing to congruence adapted frames: $\Omega_{i j}=\omega_{i j}=K_{[i j]}=-\epsilon_{i j k} H^{k} / 2$, so that the Ricci tensor comes in terms of the three GEM fields: $\vec{G}, \vec{H}$ and $K_{(i j)}$. These read

$$
\begin{aligned}
R_{\hat{0} \hat{0}}= & -\tilde{\nabla} \cdot \vec{G}+\vec{G}^{2}+\frac{1}{2} \vec{H}^{2}-\dot{\theta}-K^{(\hat{i} \hat{j})} K_{(\hat{i} \hat{j})} ; \\
R_{\hat{0} \hat{i}}= & \tilde{\nabla}^{\hat{j}} K_{(\hat{j} \hat{i})}-\theta_{\hat{i}}+\frac{1}{2}(\tilde{\nabla} \times \vec{H})_{\hat{i}}-(\vec{G} \times \vec{H})_{\hat{i}} ; \\
R_{\hat{i} \hat{j}}= & \tilde{R}_{\hat{i} \hat{j}}+\tilde{\nabla}_{\hat{i}} G_{\hat{j}}-G_{\hat{i}} G_{\hat{j}}+\dot{K}_{(\hat{i} \hat{j})}+K_{(\hat{i} \hat{j})} \theta \\
& +\frac{1}{2}\left[\dot{H}_{\hat{i} \hat{j}}+H_{\hat{i} \hat{j}} \theta+\vec{H}^{2} \delta_{\hat{i} \hat{j}}-H_{\hat{i}} H_{\hat{j}}+K_{(\hat{i} \hat{l})} H_{\hat{j}}^{\hat{l}}-H_{\hat{i}}^{\hat{l}} K_{(\hat{l} j)}\right],
\end{aligned}
$$

where $H_{i j}=\epsilon_{i j k} H^{k}$ is the dual of $\vec{H}$, and $\tilde{R}_{\hat{i} \hat{j}} \equiv \tilde{R}_{\hat{i} \hat{l} \hat{j}}$ is the Ricci tensor associated to $\tilde{R}_{\hat{i} \hat{j} \hat{k} \hat{l}}$; this tensor is not symmetric in the general case of a congruence possessing both vorticity and shear. Using $T^{\hat{0} \hat{0}}=\rho$ and $T^{\hat{0} \hat{i}}=J^{\hat{i}}$, the time-time, time-space, and space-space components of the Einstein field equations with sources, Eq. (14), read, respectively:

$$
\begin{aligned}
\tilde{\nabla} \cdot \vec{G}= & -4 \pi\left(2 \rho+T_{\alpha}^{\alpha}\right)+\vec{G}^{2}+\frac{1}{2} \vec{H}^{2}-\dot{\theta}-K^{(\hat{i} \hat{j})} K_{(\hat{i} \hat{j})} ; \\
\tilde{\nabla} \times \vec{H}= & -16 \pi \vec{J}+2 \vec{G} \times \vec{H}+2 \tilde{\nabla} \theta-2 \tilde{\nabla}_{\hat{j}} K^{(\hat{j} \hat{i})} \vec{e}_{\hat{i}} ; \\
8 \pi\left(T_{\hat{i} \hat{j}}-\frac{1}{2} \delta_{\hat{i} \hat{j}} T_{\alpha}^{\alpha}\right)= & \tilde{R}_{\hat{i} \hat{j}}+\tilde{\nabla}_{\hat{i}} G_{\hat{j}}-G_{\hat{i}} G_{\hat{j}}+\dot{K}_{(\hat{i} \hat{j})}+K_{(\hat{i} \hat{j})} \theta \\
& +\frac{1}{2}\left[\dot{H}_{\hat{i} \hat{j}}+H_{\hat{i} \hat{j}} \theta+\vec{H}^{2} \delta_{\hat{i} \hat{j}}-H_{\hat{i}} H_{\hat{j}}+K_{(\hat{i} \hat{l})} H_{\hat{j}}^{\hat{l}}-H_{\hat{i}}{ }^{\hat{l}} K_{(\hat{l} \hat{j})}\right] .
\end{aligned}
$$

Eqs. (99)-(100) are the gravitational analogues of the electromagnetic equations (86) and (87), respectively; Eq. (101) has no electromagnetic counterpart.

As for the algebraic Bianchi identities (14), using (90)-92), the time-time (equal to spacespace, as discussed in Sec. 2.1.2, time-space and space-time components become, respectively:

$$
\begin{aligned}
\tilde{\nabla} \cdot \vec{H} & =-\vec{G} \cdot \vec{H} ; \\
\tilde{\nabla} \times \vec{G} & =-\dot{\vec{H}}-\vec{H} \theta+H_{\hat{j}} K^{(\hat{i} \hat{j})} \vec{e}_{\hat{i}} ; \\
K_{(\hat{i} \hat{j})} H^{\hat{j}} & =-\star \tilde{R}_{\hat{j} \hat{j}}^{\hat{j}} .
\end{aligned}
$$

Eqs. 102-103 are the gravitational analogues of the time and space projections of the electromagnetic Bianchi identities, Eqs. 88)-89, respectively ${ }^{17}$ Eq. (104) has no electromagnetic analogue.

\footnotetext{
${ }^{16}$ This is manifest in the algebraic Bianchi identities. The generalization of Eq. 104 for non-congruence adapted frames is $\star \tilde{R}_{j i}^{j}=2 \epsilon_{i k j} \omega^{j} \Omega^{k}-2 K_{(i k)} \omega^{k}$; the first term is not zero in general when $\vec{\Omega} \neq \vec{\omega}$ and/or both $K_{(i j)}$ and $\omega^{k}$ are different from zero.

${ }^{17}$ Eqs. 102 - 103 are equivalent to Eqs. (7.3) of [27; therein they are obtained through a different procedure, not by projecting the identity $\star R_{\gamma \beta}^{\gamma \alpha}=0 \Leftrightarrow R_{[\alpha \beta \gamma] \delta}=0$, but instead from the splitting of the identity $d^{2} \mathbf{u}=$ $0 \Leftrightarrow u_{[\alpha ; \beta \gamma]}=0$. Noting that $u_{[\alpha ; \beta \gamma]}=-R_{[\alpha \beta \gamma] \lambda} u^{\lambda}$, we see that the latter is indeed encoded in the time-time and space-time parts (with respect to $u^{\alpha}$ ) of the former.
} 
This equation states that if the observer congruence has both vorticity and shear/expansion then $\tilde{R}_{i j k l}$ does not obey the algebraic Bianchi identities for a 3D curvature tensor.

Note this remarkable aspect: all the terms in the Maxwell equations (86), (88) and (89) have a gravitational counterpart in 99,102 and 103 , respectively, substituting $\{\vec{E}, \vec{B}\} \rightarrow\{\vec{G}, \vec{H}\}$ (up to some numerical factors). As for (87), there are clear gravitational analogues in 100 to the terms $\vec{G} \times \vec{B}$ and the current $4 \pi \vec{j}$, but not to the remaining terms. It should nevertheless be noted that, as shown in Sec. 5 below, in the post-Newtonian regime (or in the "GEM limit" of linearized theory), the term $2 \tilde{\nabla} \theta$ of 100 embodies a contribution analogous to the displacement current term $\dot{\vec{E}}$ of 87 . The gravitational equations in turn contain, as one might expect, terms with no parallel in electromagnetism, most of them involving the shear/expansion tensor $K_{(\alpha \beta)}$.

Eqs. (99)-104) are the inertial fields based version of the tidal tensor equations (19)-22) of Sec. 2. Finally, it is also worth mentioning that these equations have been expressed in tetrad formalism also in the literature, e.g. [36, 46], albeit in a different language; we note that, for congruence adapted frames $(\vec{\Omega}=\vec{\omega})$, zero cosmological constant, and perfect fluids, Eqs. (6.45)-(6.47) of [36] correspond, respectively, to Eqs. (102)-(104) above; and (6.50)-(6.51) therein to (99)-(100).

\subsubsection{Special cases: quasi-Maxwell regime ( $1+3$ formalism), and hypersurface or- thogonal observers $(3+1$ formalism)}

Eqs. (99)-(104) encompass two notable regimes in the literature: (i) the "quasi-Maxwell" regime of Sec. 3.2.2, corresponding to stationary fields, and a frame adapted to a rigid congruence of stationary observers, which is obtained by setting $K_{(\alpha \beta)}$ and all time derivatives to zero; and (ii) the case of a frame adapted to an hypersurface orthogonal (i.e., vorticity free) congruence, obtained by setting $\vec{H}=0$ in the equations above, leading to what is sometimes dubbed the " $3+1$ " splitting, which is closely related to the well known ADM formalism (e.g. [108, 107, 52]). Note that these special limits correspond to the ones equally labeled (i) and (ii) in Sec. 3.2.1. Let us start by case (i), also known as the " $1+3$ formalism" (e.g. [24]) or threading picture [27, 28] for stationary spacetimes, which is where the similarity with the electromagnetism gets closer, since, as we have seen in the previous section, most of the differing terms between the gravitational field Eqs. (99), 100$),(102),(103)$, and their electromagnetic counterparts $(86)-(89)$, involve $K_{(\alpha \beta)}$. The field equations in this regime are given in Table 2. Therein we drop the hats in the indices; as discussed in Sec. 3.2.1 (notable case (ii)), in this regime one can identify the quotient space with a 3-D Riemannian manifold $(\Sigma, \mathbf{h})$ whose metric $h_{i j}$ measures the fixed distance between neighboring observers. The indices $i, j, \ldots$ are raised and lowered by $h_{i j}, \vec{G}$ and $\vec{H}$ can be interpreted as vector fields on $(\Sigma, \mathbf{h})$, the derivatives $\tilde{\nabla}_{i}$ become the usual covariant derivatives with respect to $h_{i j}$, and $\tilde{R}_{i j}$ is its Ricci tensor. The gravitational field equations in Table 2 exhibit a striking similarity with their electromagnetic counterparts, in spite of some natural differences that remain - numerical factors, the source and quadratic terms in $(2,4 \mathrm{~b})$ with no electromagnetic counterpart. We note in particular that, by simply replacing $\{\vec{E}, \vec{B}\} \rightarrow\{\vec{G}, \vec{H}\}$ in $(2.5 \mathrm{a})-(2,8 \mathrm{a})$, one obtains, up to some numerical factors, Eqs. (2.5b), (2.7b)-(2.8b). Of course, the electromagnetic terms involving products of GEM fields with EM fields, are mimicked in gravity by second order terms in the GEM fields. This is intrinsic to the non-linear nature of the gravitational field, and may be thought of as manifesting the fact that the gravitational field sources itself. It is interesting to note in this context that the term $2 \vec{G} \times \vec{H}$ in Eq. 2.5b), sourcing the curl of the gravitomagnetic field, 
Table 2: The gravito-electromagnetic analogy based on inertial GEM fields.

Electromagnetism

Lorentz Force:

$\left(\nabla_{\mathbf{U}} \mathbf{U}\right)^{\hat{i}}=\frac{q}{m}\left[U^{\hat{0}} E^{\hat{i}}+(\vec{U} \times \vec{B})^{\hat{i}}\right]$

Precession of magnetic dipole:

$$
\frac{D \vec{S}}{d \tau}=\vec{\mu} \times \vec{B}
$$

Gravity

Geodesic Equation $(\vec{H}=\vec{\Omega}+\vec{\omega})$ :

2.1a) $\frac{\tilde{D} \vec{U}}{d \tau}=U^{\hat{0}}\left[U^{\hat{0}} \vec{G}+\vec{U} \times \vec{H}-\sigma_{\hat{j}}^{\hat{i}} U^{\hat{j}} \mathbf{e}_{\hat{i}}-\frac{1}{3} \theta \vec{U}\right]$

$2.1 \mathrm{~b})$

Stationary fields, rigid, congruence adapted frame: $\vec{\Omega}=\vec{\omega}=\vec{H} / 2$ (quasi-Maxwell formalism)

Force on magnetic dipole:

$$
\vec{F}_{\mathrm{EM}}=\tilde{\nabla}(\vec{B} \cdot \vec{\mu})-\frac{1}{2} \vec{\mu}(\tilde{\nabla} \cdot \vec{B})-\frac{1}{2}(\vec{\mu} \cdot \vec{H}) \vec{E}
$$

Force on gyroscope:

22.3a) $\quad \vec{F}_{\mathrm{G}}=\frac{1}{2}[\tilde{\nabla}(\vec{H} \cdot \vec{S})-\vec{S}(\tilde{\nabla} \cdot \vec{H})-2(\vec{S} \cdot \vec{H}) \vec{G}]$
Maxwell Source Equations

$$
F_{; \beta}^{\alpha \beta}=4 \pi j^{\alpha}
$$

- Time Component:

$\tilde{\nabla} \cdot \vec{E}=4 \pi \rho_{c}+\vec{H} \cdot \vec{B}$

- Space Components:

$\tilde{\nabla} \times \vec{B}=\vec{G} \times \vec{B}+4 \pi \vec{j}$

No electromagnetic analogue
Einstein Equations

$R_{\mu \nu}=8 \pi\left(T_{\mu \nu}-\frac{1}{2} g_{\mu \nu} T_{\alpha}^{\alpha}\right)$

- Time-Time Component:

24 4a) $\quad \tilde{\nabla} \cdot \vec{G}=-4 \pi\left(2 \rho+T_{\alpha}^{\alpha}\right)+\vec{G}^{2}+\frac{1}{2} \vec{H}^{2}$

(2.4b)

- Time-Space Components:

$\tilde{\nabla} \times \vec{H}=2 \vec{G} \times \vec{H}-16 \pi \vec{J}$

- Space-Space Component:

$$
\tilde{\nabla}_{i} G_{j}-G_{i} G_{j}+\frac{1}{2} \vec{H}^{2} h_{i j}+\tilde{R}_{i j}=8 \pi\left(\frac{1}{2} h_{i j} T_{\alpha}^{\alpha}+T_{i j}\right)
$$

(2.5a)
Bianchi Identity

$\star F_{; \beta}^{\alpha \beta}=0 \quad\left(\Leftrightarrow F_{[\alpha \beta ; \gamma]}=0\right)$

- Time Component:

$\tilde{\nabla} \cdot \vec{B}=-\vec{H} \cdot \vec{E}$

- Space Components:

$\tilde{\nabla} \times \vec{E}=\vec{G} \times \vec{E}$
Algebraic Bianchi Identity

$\star R_{\gamma \beta}^{\gamma \alpha}=0 \quad\left(\Leftrightarrow R_{[\alpha \beta \gamma] \delta}=0\right)$

- Time-Time (or Space-Space) Component:

$$
\tilde{\nabla} \cdot \vec{H}=-\vec{H} \cdot \vec{G}
$$

- Space-Time Components:

$$
\tilde{\nabla} \times \vec{G}=0
$$

resembles the electromagnetic Poynting vector $\vec{p}_{E M}=\vec{E} \times \vec{B} / 4 \pi$; and the contribution $\vec{G}^{2}+\vec{H}^{2} / 2$ in Eq. (2.5b), sourcing the divergence of the gravitoelectric field, resembles the electromagnetic energy density $\rho_{E M}=\left(\vec{E}^{2}+\vec{B}^{2}\right) / 8 \pi$. For these reasons these quantities are dubbed in e.g. [19, 23, 24] gravitational "energy density" and "energy current density", respectively. It is also interesting that, in the asymptotic limit, $\vec{p}_{G} \equiv-\vec{G} \times \vec{H} / 4 \pi$ corresponds to the time-space components of the LandauLifshitz [18] pseudo-tensor $t^{\mu \nu}$ [61]. One should however bear in mind that, by contrast with their electromagnetic counterparts, these quantities are artifacts of the reference frame, with no physical significance from a local point of view (see related discussion in Sec. 6.1).

Let us turn now to case (ii); taking a vorticity-free congruence of observers (i.e., $\vec{\omega}=\vec{H}=0$ ), 
the Einstein Eqs. (99)-(101) can be written as, respectively,

$$
\begin{aligned}
16 \pi \rho & =\tilde{R}+\theta^{2}-K^{(\hat{i} \hat{j})} K_{(\hat{i} \hat{j})} ; \\
8 \pi \vec{J} & =\tilde{\nabla} \theta-\tilde{\nabla}_{\hat{j}} K^{(\hat{j} \hat{i})} \vec{e}_{\hat{i}} ; \\
\dot{K}_{(\hat{i} \hat{j})} & =G_{\hat{i}} G_{\hat{j}}-\tilde{\nabla}_{\hat{i}} G_{\hat{j}}-\tilde{R}_{i j}-\theta K_{(\hat{i} \hat{j})}+8 \pi\left(T_{\hat{i} \hat{j}}-\frac{1}{2} \delta_{\hat{i} \hat{j}} T_{\alpha}^{\alpha}\right) .
\end{aligned}
$$

This regime corresponds to case (i) discussed in Sec. 3.2.1 thus $\tilde{\nabla}_{i}$ are covariant derivatives with respect to the metric $h_{i j}$ induced on the hypersurfaces $\Sigma_{t}$ orthogonal to $u^{\alpha}$, and $\tilde{R}$ and $\tilde{R}_{i j}$ are, respectively, their 3-D Ricci scalar and tensor. Eq. (105) is known in the framework of the ADM formalism [108, 107, 109] as the "Hamiltonian constraint". Since this equation is the tetrad timetime component of Eq. (14), it can either be directly obtained from the latter by noting that, when $\omega^{\alpha}=0, K_{(\alpha \beta)}$ is the extrinsic curvature of the hypersurfaces orthogonal to the congruence, and employing the scalar Gauss equation (e.g. Eq. (2.95) of [107]); or from Eq. (99) above, computing $R=R_{\hat{i}}^{\hat{i}}-R_{\hat{0} \hat{0}}$ from Eqs. 296, , 97 (with $\vec{H}=\overrightarrow{0}$ ), substituting into 99 , and then using 14 a) to eliminate $R$. Eq. 106 follows directly from Eq. 100 by making $\vec{H}=\overrightarrow{0}$, and is known as the "momentum constraint" [107, 109]. The space-space Eq. (107) is the dynamical equation for the extrinsic curvature. It can be put in the usual forms in the literature as follows: 1) noting from Eq. (3.17) of [107] that $G_{\alpha}=-(1 / N) \tilde{\nabla}_{\alpha} N$, where $N$ is the lapse function; 2) noting that, since $\Gamma_{\hat{0} \hat{j}}^{\hat{i}}=\Omega^{\hat{i}}{ }_{\hat{j}}=0, \dot{K}_{(\hat{i} \hat{j})}=\nabla_{\mathbf{u}} K_{(\hat{i} \hat{j})}$, and then using ${ }^{18}$ Eq. (3.42) of [107] to express this in terms of the Lie derivative $\mathcal{L}_{\mathbf{m}} K_{(\hat{i} \hat{j})}$ along the "normal evolution vector" $\mathbf{m}=N \mathbf{u}$, obtaining Eq. (4.17) of [107], or in terms of $\mathcal{L}_{\mathbf{m}+\boldsymbol{\beta}} K_{(\hat{i} \hat{j})}$, for some suitable spatial "shift" vector $\boldsymbol{\beta}$ ( $\left.\beta^{\alpha} u_{\alpha}=0\right)$, leading to the best known "ADM" form Eq. (4.64) of [107]. Eqs. (105)-(106) have little resemblance to their physical electromagnetic counterparts 86 - 87) (for $\vec{H}=\vec{B}=\overrightarrow{0}$ ); but in this framework a different (purely formal) analogy is sometimes drawn (e.g. [109]): a parallelism between Eqs. (105)-106) and the two electromagnetic constraints (for Lorentz frames in flat spacetime) $\partial_{i} E^{i}=4 \pi \rho_{c}$ and $\partial_{i} B^{i}=0$, and between the $\mathrm{ADM}$ evolution equations for $K_{(i j)}$ and for the spatial metric, written in a coordinate system adapted to the foliation (e.g. Eqs. (4.63)-(4.64) of [107]), and the dynamical equations for the curls of $\vec{B}$ and $\vec{E}$.

\subsection{Relation with tidal tensor formalism}

The analogy based on the gravito-electromagnetic fields $\vec{G}$ and $\vec{H}$ is intrinsically different from the gravito-electromagnetic analogy based on tidal tensors of Sec. 2; the latter stems from tensor equations, whereas the former are fields of inertial forces, i.e., artifacts of the reference frame. A relationship between the two formalisms exists nevertheless, as in an arbitrary frame one can express the gravitational tidal tensors in terms of the GEM fields, using the expressions for the tetrad components of Riemann tensor Eqs. (90)-(91). This relationship is in many ways illuminating, as we shall see; it is one of the main results in this work, due the importance of using the two formalisms together in practical applications, to be presented elsewhere (e.g. [29]). In an arbitrary frame one can express the gravitational tidal tensors in terms of the GEM fields, using the expressions for the tetrad components of Riemann tensor (90)-(92). The expressions obtained are to be compared with the analogous electromagnetic situation, i.e., the electromagnetic tidal tensors computed from

\footnotetext{
${ }^{18}$ Our conventions relate to the ones in [107] by identifying our $\left\{K_{(i j)}, \theta\right\}$ with $\left\{-K_{i j},-K\right\}$ therein.
} 
the fields as measured in an arbitrarily accelerating, rotating, and shearing frame (in flat or curved spacetime).

We start by the electromagnetic tidal tensors; using the abbreviated notation $E_{\alpha \beta} \equiv\left(E^{u}\right)_{\alpha \beta}=$ $F_{\alpha \gamma ; \beta} u^{\gamma}, B_{\alpha \beta} \equiv\left(B^{u}\right)_{\alpha \beta}=\star F_{\alpha \gamma ; \beta} u^{\gamma}$, cf. Table 1, it follows that

$$
E_{\alpha \gamma}=E_{\alpha ; \gamma}-F_{\alpha \beta} u_{; \gamma}^{\beta} ; \quad B_{\alpha \gamma}=B_{\alpha ; \gamma}-\star F_{\alpha \beta} u_{; \gamma}^{\beta} .
$$

Using decompositions (1), and Eq. (75), we obtain the tetrad components $\left(E_{\hat{0} \hat{i}}=B_{\hat{0} \hat{i}}=0\right)$ :

$$
\begin{aligned}
E_{\hat{i} \hat{j}} & =\tilde{\nabla}_{\hat{j}} E_{\hat{i}}-\epsilon_{\hat{i}}^{\hat{l} \hat{m}} B_{\hat{m}} K_{\hat{l} \hat{j}} ; \\
B_{\hat{i} \hat{j}} & =\tilde{\nabla}_{\hat{j}} B_{\hat{i}}+\epsilon_{\hat{i}}^{\hat{l} \hat{m}} E_{\hat{m}} K_{\hat{l} \hat{j}} ; \\
E_{\hat{i} \hat{0}} & =\dot{E}_{\hat{i}}+(\vec{\Omega} \times \vec{E})_{\hat{i}}+(\vec{G} \times \vec{B})_{\hat{i}} \\
B_{\hat{i} \hat{0}} & =\dot{B}_{\hat{i}}+(\vec{\Omega} \times \vec{B})_{\hat{i}}-(\vec{G} \times \vec{E})_{\hat{i}},
\end{aligned}
$$

or, using $K_{i j}=\omega_{i j}+K_{(i j)}$, and choosing a congruence adapted frame $(\vec{\omega}=\vec{\Omega}=\vec{H} / 2)$,

$$
\begin{aligned}
E_{\hat{i} \hat{j}} & =\tilde{\nabla}_{\hat{j}} E_{\hat{i}}-\frac{1}{2}\left[\vec{B} \cdot \vec{H} \delta_{\hat{i} \hat{j}}-B_{\hat{j}} H_{\hat{i}}\right]-\epsilon_{\hat{i}}^{\hat{l} \hat{m}} B_{\hat{m}} K_{(\hat{l} \hat{j})} \\
B_{\hat{i} \hat{j}} & =\tilde{\nabla}_{\hat{j}} B_{\hat{i}}+\frac{1}{2}\left[\vec{E} \cdot \vec{H} \delta_{\hat{i} \hat{j}}-E_{\hat{j}} H_{\hat{i}}\right]+\epsilon_{\hat{i}}^{\hat{l} \hat{m}} E_{\hat{m}} K_{(\hat{l} \hat{j})} \\
E_{\hat{i} \hat{0}} & =\dot{E}_{\hat{i}}+\frac{1}{2}(\vec{H} \times \vec{E})_{\hat{i}}+(\vec{G} \times \vec{B})_{\hat{i}} \\
B_{\hat{i} \hat{0}} & =\dot{B}_{\hat{i}}+\frac{1}{2}(\vec{H} \times \vec{B})_{\hat{i}}-(\vec{G} \times \vec{E})_{\hat{i}} .
\end{aligned}
$$

Let us compute the gravitational tidal tensors. From the definitions of $\mathbb{E}_{\alpha \beta}$ and $\mathbb{H}_{\alpha \beta}$ in Table 1 . and using the tetrad components of the Riemann tensor, Eqs. 90$)-(91)$, we obtain $\left(\mathbb{E}_{\hat{0} \hat{\alpha}}=\mathbb{E}_{\hat{\alpha} \hat{0}}=\right.$ $\left.\mathbb{H}_{\hat{0} \hat{\alpha}}=\mathbb{H}_{\hat{\alpha} \hat{0}}=0\right)$ :

$$
\begin{aligned}
\mathbb{E}_{\hat{i} \hat{j}} & =-\tilde{\nabla}_{\hat{j}} G_{\hat{i}}+G_{\hat{i}} G_{\hat{j}}-\dot{K}_{\hat{i} \hat{j}}+K_{\hat{l} \hat{j}} \Omega_{\hat{i}}^{\hat{l}}+\Omega_{\hat{j}}^{\hat{l}} K_{\hat{i} \hat{l}}-K_{\hat{j}^{\hat{l}}} K_{\hat{i} \hat{l}} ; \\
\mathbb{H}_{\hat{i} \hat{j}} & =-\tilde{\nabla}_{\hat{j}} \omega_{\hat{i}}+\delta_{\hat{i} \hat{j}} \tilde{\nabla} \cdot \vec{\omega}+2 G_{\hat{j}} \omega_{\hat{i}}+\epsilon_{\hat{i}}^{\hat{l} \hat{m}} \tilde{\nabla}_{\hat{l}} K_{(\hat{j} \hat{m})} .
\end{aligned}
$$

For a congruence adapted frame these expressions become:

$$
\begin{aligned}
\mathbb{E}_{\hat{i} \hat{j}}= & -\tilde{\nabla}_{\hat{j}} G_{\hat{i}}+G_{\hat{i}} G_{\hat{j}}+\frac{1}{4}\left(\vec{H}^{2} \delta_{\hat{i} \hat{j}}-H_{\hat{j}} H_{\hat{i}}\right)+\frac{1}{2} \epsilon_{\hat{i} \hat{j} \hat{k}} \dot{H}^{\hat{k}}+\epsilon_{\hat{j} \hat{m}}^{\hat{l}} H^{\hat{m}} K_{(\hat{i} \hat{l})} \\
& -\dot{K}_{(\hat{i} \hat{j})}-\delta^{\hat{l} \hat{m}} K_{(\hat{i} \hat{l})} K_{(\hat{m} \hat{j})} \\
\mathbb{H}_{\hat{i} \hat{j}}= & -\frac{1}{2}\left[\tilde{\nabla}_{\hat{j}} H_{\hat{i}}+(\vec{G} \cdot \vec{H}) \delta_{\hat{i} \hat{j}}-2 G_{\hat{j}} H_{\hat{i}}\right]+\epsilon_{\hat{i}}^{\hat{l} \hat{m}} \tilde{\nabla}_{\hat{l}} K_{(\hat{j} \hat{m})} .
\end{aligned}
$$

In 119 we substituted $\tilde{\nabla} \cdot H=-\vec{G} \cdot \vec{H}$ using Eq. 102. Note the formal similarities with the electromagnetic analogues 112)-113). All the terms present in $E_{i j}$ and $B_{i j}$, except for the last term of the latter, have a correspondence in their gravitational counterparts $\mathbb{E}_{i j}, \mathbb{H}_{i j}$, substituting $\{\vec{E}, \vec{B}\} \rightarrow-\{\vec{G}, \vec{H}\}$ and correcting some factors of 2 . However, the gravitational tidal tensors contain additional terms, which (together with the differing numerical factors) encode the crucial differences in the tidal dynamics of the two interactions. The fourth and fifth terms in (118) have the role of canceling out the antisymmetric part of $\tilde{\nabla}_{\hat{j}} G_{\hat{i}}$, that is, canceling out the contribution of 
the curl of $\vec{G}$ to the gravitoelectric tidal tensor, as can be seen from Eq. (103). Note in particular the term involving $\dot{H}^{i}$, which has no counterpart in the electric tidal tensor (112); in Eq. (103), that term shows up "inducing" the curl of $\vec{G}$, in a role analogous to $\dot{B}^{i}$ in the equation 89 for $\widetilde{\nabla} \times \vec{E}$, which might lead one to think about gravitational induction effects in analogy with Faraday's law of electromagnetism. The fact that it is being subtracted in (118), means, however, that the curl of $\vec{G}$ does not translate into physical, covariant forces. For instance, it does not induce rotation in a set of free neighboring particles (see Eq. (26) above and discussion therein), nor does it torque an extended rigid body, as shown in the companion paper [6].

There are some interesting special regimes where the relation between the tidal tensor and the inertial fields formalism becomes simpler. One is the "quasi-Maxwell" regime of Sec. 3.2.2, i.e., stationary spacetimes, and a frame adapted to a rigid (i.e., shear and expansion-free) congruence of stationary observers. The gravitational tidal tensors as measured in such frame can be expressed entirely in terms of the gravitoelectric $\vec{G}$ and gravitomagnetic $\vec{H}$ fields; the non-vanishing components are:

$$
\begin{aligned}
\mathbb{E}_{i j} & =-\tilde{\nabla}_{j} G_{i}+G_{i} G_{j}+\frac{1}{4}\left(\vec{H}^{2} h_{i j}-H_{j} H_{i}\right) ; \\
\mathbb{H}_{i j} & =-\frac{1}{2}\left[\tilde{\nabla}_{j} H_{i}+(\vec{G} \cdot \vec{H}) h_{i j}-2 G_{j} H_{i}\right] .
\end{aligned}
$$

The hats in the indices of these expressions are dropped (as we did in Table 2 because, as discussed in Secs. 3.2.1 and 3.4.3, in this regime $h_{i j}$ is a canonical metric on the quotient space, whose LeviCivita connection is $\tilde{\nabla}_{j}$; so the $i, j$ (raised and lowered by $h_{i j}$ ) may refer to an arbitrary (possibly coordinate) basis in this manifold, not necessarily tetrad components.

The non-vanishing components of the electromagnetic tidal tensors are, under the same conditions,

$$
\begin{aligned}
& E_{i j}=\tilde{\nabla}_{j} E_{i}-\frac{1}{2}\left[\vec{B} \cdot \vec{H} h_{i j}-B_{j} H_{i}\right] \quad \text { (a) } \quad E_{i 0}=\frac{1}{2}(\vec{H} \times \vec{E})_{i}+(\vec{G} \times \vec{B})_{i} \\
& B_{i j}=\tilde{\nabla}_{j} B_{i}+\frac{1}{2}\left[\vec{E} \cdot \vec{H} h_{i j}-E_{j} H_{i}\right] \quad \text { (a) } \quad B_{i 0}=\frac{1}{2}(\vec{H} \times \vec{B})_{i}-(\vec{G} \times \vec{E})_{i}
\end{aligned}
$$

Thus again, even in the stationary regime, the electromagnetic tidal tensors have non-vanishing time components, unlike their gravitational counterparts. The spatial parts, however, are very similar in form; note that replacing $\{\vec{E}, \vec{B}\} \rightarrow-\{\vec{G}, \vec{H} / 2\}$ in 123 , the time components vanish, and one almost obtains the space part (121), apart from the factor of 2 in the third term; and that a similar substitution in 122 almost leads to 120$)$, apart from the term $G_{i} G_{j}$, which has no electromagnetic counterpart. The gravitational and electromagnetic tidal tensors are nevertheless very different, even in this regime; namely in their symmetries. $E_{i j}$ is not symmetric, whereas $\mathbb{E}_{i j}$ is (the second and third terms in (120) are obviously symmetric; and that the first one also is can be seen from Eq. (2,8b) of Table 2). As for the magnetic tidal tensors, note that, by virtue of Eq. (2.5b), the last term of (121) ensures that, in vacuum, the antisymmetric part $H_{[i ; j]}$ (i.e., the curl of $\vec{H}$ ) is subtracted from $H_{i ; j}$ in 117 , thus keeping $\mathbb{H}_{i j}$ symmetric, by contrast with $B_{i j}$. This can be seen explicitly by noting that in vacuum (121) can be put in the equivalent form

$$
\mathbb{H}_{i j}=-\frac{1}{2}\left[H_{i ; j}-H_{[i ; j]}+(\vec{G} \cdot \vec{H}) h_{i j}-2 G_{(j} H_{i)}\right] \text {, }
$$


where we used $H_{[i ; j]}=2 G_{[j} H_{i]}$, as follows from Eq. (2.5b).

Another interesting regime to consider is the weak field limit, where the non-linearities of the gravitational field are negligible, and compare with electromagnetism in inertial frames. From Eqs. (112)-(115), the non-vanishing components of the electromagnetic tidal tensors measured by observers at rest in an inertial frame are:

$$
E_{i j}=E_{i, j} ; \quad E_{i 0}=\dot{E}_{i} ; \quad B_{i j}=B_{i, j} ; \quad B_{i 0}=\dot{B}_{i}
$$

i.e., they reduce to ordinary derivatives of the electric and magnetic fields. The linearized gravitational tidal tensors are, from Eqs. (118)- 119 :

$$
\mathbb{E}_{i j} \approx-G_{i, j}+\frac{1}{2} \epsilon_{i j k} \dot{H}^{k}-\dot{K}_{(i j)} ; \quad\left(\text { a) } \quad \mathbb{H}_{i j} \approx-\frac{1}{2} H_{i, j}+\epsilon_{i}^{l m} K_{(j m), l}\right.
$$

Thus, even in the linear regime, the gravitational tidal tensors cannot, in general, be regarded as derivatives of the gravitoelectromagnetic fields $\vec{G}$ and $\vec{H}$. Noting, from Eq. 137) below, that $K_{(i j)}$ is the time derivative of the spatial metric, we see that only if the fields are time independent in the chosen frame do we have $\mathbb{E}_{i j} \approx-G_{i, j}, \mathbb{H}_{i j} \approx-\frac{1}{2} H_{i, j}$.

\subsection{Force on a gyroscope}

In the framework of the inertial GEM fields, there is also an analogy relating the gravitational force on a gyroscope and the electromagnetic force on a magnetic dipole. This is different from the analogy based on tidal tensors, and not as general. We start with equations (1.2) of Table 1 . which tell us that the forces are determined by the magnetic/gravitomagnetic tidal tensors as seen by the particle. For the spatial part of the forces, only the space components of the tidal tensors, as measured in the particle's proper frame, contribute. Comparing Eqs. (113) and (119), which yield the tidal tensors in terms of the electromagnetic/gravitoelectromagnetic fields, we see that a close formal analogy is possible only when $K_{(\alpha \beta)}=0$ in the chosen frame. Thus, a close analogy between the forces in this formalism can hold only when the particle is at rest with respect to a congruence for which $K_{(\alpha \beta)}=0$; that is, a rigid congruence. The rigidity requirement can be satisfied only in special spacetimes [99]; it is ensured in the "quasi-Maxwell" regime — that is, stationary spacetimes, and congruences tangent to time-like Killing vector fields therein.

Let us start by the electromagnetic problem - a magnetic dipole at rest in a rigid, but arbitrarily accelerating and rotating frame. Since the dipole is at rest in that frame, we have $\mu^{\alpha}=\left(0, \mu^{i}\right)$; hence the spatial part of the force is $F_{E M}^{i}=B^{j i} \mu_{j}$. Substituting $(123 \mathrm{a})$ in this expression yields the force exerted on the dipole, in terms of the electric and magnetic fields as measured in this frame:

$$
\vec{F}_{E M}=\tilde{\nabla}(\vec{B} \cdot \vec{\mu})+\frac{1}{2}[\vec{\mu}(\vec{E} \cdot \vec{H})-(\vec{\mu} \cdot \vec{H}) \vec{E}] .
$$

Using $\vec{H} \cdot \vec{E}=-\tilde{\nabla} \cdot \vec{B}$, cf. Eq. 2 .7a), we can re-write this expression as

$$
\vec{F}_{E M}=\tilde{\nabla}(\vec{B} \cdot \vec{\mu})-\frac{1}{2}[\vec{\mu}(\tilde{\nabla} \cdot \vec{B})+(\vec{\mu} \cdot \vec{H}) \vec{E}] .
$$

Consider now the analogous gravitational situation: a gyroscope at rest (i.e., with zero 3velocity, $U^{i}=0$ ) with respect to stationary observers (arbitrarily accelerated and rotating) in a 
stationary gravitational field. If the Mathisson-Pirani condition is employed (see [6] for details), the force exerted on it is described by Eq. (1.2b) of Table 1; using (121) we write this force in terms of the GEM fields:

$$
\vec{F}_{G}=\frac{1}{2}[\tilde{\nabla}(\vec{H} \cdot \vec{S})+\vec{S}(\vec{G} \cdot \vec{H})-2(\vec{S} \cdot \vec{H}) \vec{G}] .
$$

From Eq. $(2$.7b) we have $\vec{G} \cdot \vec{H}=-\tilde{\nabla} \cdot \vec{H}$; substituting yields [20]:

$$
\vec{F}_{G}=\frac{1}{2}[\tilde{\nabla}(\vec{H} \cdot \vec{S})-\vec{S}(\tilde{\nabla} \cdot \vec{H})-2(\vec{S} \cdot \vec{H}) \vec{G}] .
$$

Note that replacing $\{\vec{\mu}, \vec{E}, \vec{B}\} \rightarrow\{\vec{S}, \vec{G}, \vec{H} / 2\}$ in Eq. 125 one almost obtains 127, except for a factor of 2 in the last term. The last term of (127)-(128), in this framework, can be interpreted as the "weight" of the dipole's energy [20]. It plays, together with Eq. (2,5b), a crucial role in the dynamics, as it cancels out the contribution of the curl of $\vec{H}$ to the force, ensuring that it is given by a contraction of $S^{\alpha}$ with a symmetric tensor $\mathbb{H}_{\alpha \beta}$ (see the detailed discussion in Sec. 3.5). This contrasts with the electromagnetic case, where the curl of $\vec{B}$ is manifest in $B_{\alpha \beta}$ (which has an antisymmetric part) and in the force $F_{E M}^{\alpha}$.

The expression (128) was first found in [20], where it was compared to the force on a magnetic dipole as measured in the inertial frame momentarily comoving with it, in which case the last two terms of (126) vanish; herein we add expression (126), which is its electromagnetic counterpart for analogous conditions (the frame where the particle is at rest can be arbitrarily accelerating and rotating), and shows that the analogy is even stronger.

\section{4 "Ultra-stationary" spacetimes}

Ultra-stationary spacetimes are stationary spacetimes admitting rigid geodesic time-like congruences. In the coordinate system adapted to such congruence, the metric is generically obtained by taking $\Phi=0$ in Eq. (69), leading to,

$$
d s^{2}=-\left(d t-\mathcal{A}_{i}\left(x^{k}\right) d x^{i}\right)^{2}+h_{i j}\left(x^{k}\right) d x^{i} d x^{j} .
$$

Examples of these spacetimes are the Som-Raychaudhuri metrics [78], the van Stockum interior solution [79], and the Gödel [80] spacetime; see [2] for their discussion in this context. This is an interesting class of spacetimes in the context of GEM, due to the close similarity with electrodynamics, which was explored in an earlier work [1 by one of the authors: 1) they are exactly mapped [66, 1], via the Klein-Gordon equation, into curved 3-spaces with a "magnetic" field; 2) their gravitomagnetic tidal tensor is linear [1] (just like in the case of electromagnetism), and, up to a factor, matches the covariant derivative of the magnetic field of the electromagnetic analogue. A link between these two properties was suggested ${ }^{19}$ in [1]; however, the non-vanishing gravitoelectric

\footnotetext{
${ }^{19}$ In the earlier work Refs. [1, 2] by one of the authors (to whom the exact GEM fields analogy of Sec. 3 was not yet known), it was suggested that the above mapping could be interpreted as arising from the similarity of magnetic tidal forces manifest in relations 132 . It seems, however, to be much more related to the analogy based on GEM "vector" fields manifest in Eqs. 130 and 131. Even though the exact correspondence 132 reinforces in some sense the analogy, tidal forces do not seem to be the underlying principle behind the mapping, since: i) there is no electromagnetic counterpart to the non-vanishing gravitoelectric tidal tensor $\mathbb{E}_{\alpha \beta}$; ii) the Klein-Gordon equation $\square \Phi=m^{2} \Phi$ and the Hamiltonian in Sec. IV of [1] are for a (free) monopole particle, which feels no tidal forces. Thus one would expect it to reveal coordinate artifacts such as the fields $\overrightarrow{G,} \vec{H}$, not physical tidal forces.
} 
tidal tensor (while no electric field is present in the map) was a question left unanswered. Herein, putting together the knowledge from the tidal tensor and the inertial force formalisms (Secs. 2 and 3), we revisit these spacetimes and shed new light on these issues.

Eqs. (70) yield the GEM fields corresponding to the frame adapted to the rest observers, of 4 -velocity $u^{\alpha}=\delta_{0}^{\alpha}$ (in the coordinate system of $(129)$ ). They tell us that the gravitoelectric field vanishes, $\vec{G}=0$, which is consistent with the fact that no electric field arises in the mapping above; and that the gravitomagnetic field $\vec{H}$ is linear in the metric potentials:

$$
\vec{H}=\tilde{\nabla} \times \overrightarrow{\mathcal{A}}
$$

These properties can be interpreted as follows. The fact that $\vec{G}=0$ means that the rest observers are freely falling (as their acceleration $a^{\alpha}=-G^{\alpha}$ is zero); the very special property of these spacetimes is that such geodesic congruence is rigid, i.e. has no shear/expansion, allowing the metric to be time independent in a coordinate system associated to those observers (unlike the situation in general, e.g. the Kerr or Schwarzschild spacetimes). The gravitomagnetic field, on the other hand, does not vanish in this frame, which means in this context (since the frame is congruence adapted, see Sec. 3.1 and Eq. (55)), that the congruence has vorticity. The equation of motion for a free particle in this frame, cf. Eq. (68), reduces to

$$
\frac{\tilde{D} \vec{U}}{d \tau}=U^{\hat{0}} \vec{U} \times \vec{H}
$$

similar to the equation of motion of a charged particle under the action of a magnetic field; and since $\vec{H}$ is linear in the metric, the similarity with the electromagnetic analogue is indeed close.

Let us now examine the tidal effects. This type of spacetimes have a very special property: the gravitomagnetic tidal tensor measured by the observers $u^{\alpha}=\delta_{0}^{\alpha}$ is linear in the fields (and thus in the metric potentials), cf. Eq. (121), and, just like in the electromagnetic analogue, it is given by the covariant derivative of $\vec{H}$ with respect to the spatial metric $h_{i j}$ :

$$
\mathbb{H}_{i j}=-\frac{1}{2} \tilde{\nabla}_{j} H_{i}=-\frac{1}{2} \tilde{\nabla}_{j}(\tilde{\nabla} \times \overrightarrow{\mathcal{A}})_{i}
$$

$\left(\mathbb{H}_{0 j}=\mathbb{H}_{00}=\mathbb{H}_{j 0}\right.$ for these observers $)$. This reinforces the similarity with electromagnetism. The gravitoelectric tidal tensor is, however, non-zero, as seen from Eq. (120):

$$
\mathbb{E}_{i j}=\frac{1}{4}\left(\vec{H}^{2} h_{i j}-H_{j} H_{i}\right),
$$

even though $\vec{G}=\overrightarrow{0}$. This should not be surprising, for the following reasons: i) it is always possible to make $\vec{G}$ vanish by choosing freely falling observers (this is true in an arbitrary spacetime), but that does not eliminate the tidal effects, as they arise from the curvature tensor; ii) in the case of ultrastationary spacetimes, $\mathbb{E}_{\alpha \beta}$ is actually a non-linear tensor in $\vec{H}$, which merely reflects the fact that, except on very special circumstances, $\mathbb{E}_{\alpha \beta}$ cannot be thought of as simply a covariant derivative of some gravitoelectric field $\vec{G}$.

The tidal tensor (133) exhibits other interesting properties. It vanishes along the direction of the gravitomagnetic field $H^{\alpha}$ : let $X^{\alpha}$ be a spatial vector (with respect to $u^{\alpha}, X^{\alpha} u_{\alpha}=0$ ); if it is parallel to $H^{\alpha}$, then $\mathbb{E}^{\alpha}{ }_{\beta} X^{\beta}=0$. That is, the tidal force, or the relative acceleration of two neighboring test particles of 4 -velocity $u^{\alpha}$, connected by $X^{\alpha}$, vanishes. If $X^{\alpha}$ is orthogonal to the 
gravitomagnetic field, $H^{\alpha} X_{\alpha}=0$, then it is an eigenvector of $\mathbb{E}_{\beta}^{\alpha}$, with eigenvalue $\vec{H}^{2}$. Thus, in the two dimensional subspace (on the rest space $u^{i}=0$ ) spanned by the vectors orthogonal to $H^{\alpha}$, the tidal force $-\mathbb{E}_{\beta}^{\alpha} X^{\beta}$ is proportional to the separation vectors $X^{\alpha}$. Next we will physically interpret this for the special case of the Gödel universe.

\subsection{The Gödel Universe}

The Gödel universe is a solution corresponding to an homogeneous rotating dust with negative cosmological constant. The homogeneity implies that the dust rotates around every point. The line element can be put in the form (129), with

$$
\mathcal{A}_{i} d x^{i}=e^{\sqrt{2} \omega x} d y, \quad \gamma_{i j} d x^{i} d x^{j}=d x^{2}+\frac{1}{2} e^{2 \sqrt{2} \omega x} d y^{2}+d z^{2},
$$

where $\omega$ is a constant. The gravitomagnetic field is uniform, $\vec{H}=\tilde{\nabla} \times \overrightarrow{\mathcal{A}}=2 \omega \vec{e}_{z}$; hence, by virtue of $(132)$, the gravitomagnetic tidal tensor vanishes, $\mathbb{H}_{\alpha \beta}=0$. For this reason, this universe has been interpreted in [1, 2] as being analogous to an uniform magnetic field in the curved 3-manifold with metric $\gamma_{i j}$, and the homogeneous rotation physically interpreted in analogy with a gas of charged particles subject to a uniform magnetic field - as in that case one likewise has Larmor orbits around any point.

Now we will interpret its gravitoelectric tidal tensor. In the coordinate system of 134 it reads, for the rest $\left(u^{i}=0\right)$ observers,

$$
\mathbb{E}_{i j}=\omega^{2}\left(\gamma_{i j}-\delta_{i}^{z} \delta_{j}^{z}\right)
$$

It vanishes along $z$, and is isotropic in the spatial directions $x, y$ orthogonal to $\vec{H}$. It is similar to the Newtonian tidal tensor $\partial_{i} \partial_{j} V$ of a potential $V=\omega^{2}\left(x^{2}+y^{2}\right) / 2$, corresponding to a 2-D harmonic oscillator, which is the potential of the Newtonian analogue of the Gödel Universe [81]: a uniform, infinitely long and wide cylinder of dust rotating rigidly with angular velocity $\omega$. The potential $V$ is such that the gravitational attraction exactly balances the centrifugal force on each fluid element of the rotating cylinder. The rigid rotation causes a curious effect in the Newtonian system. Consider a Cartesian coordinate system $\mathcal{S}$ with origin at the axis of rotation of the cylinder, and let $\vec{r}$ be the position vector of an arbitrary dust particle. Its equation of motion is $\dot{\vec{r}}=\vec{\omega} \times \vec{r}$. Now take a particular dust particle at position $\vec{r}_{0}$, and consider the Cartesian coordinate system $\mathcal{S}^{\prime}$ originating and comoving with it. The position vector relative to $\mathcal{S}^{\prime}$ is $\vec{r}^{\prime}=\vec{r}-\vec{r}_{0}$. Hence the equation of motion of an arbitrary dust particle with respect to $\mathcal{S}^{\prime}$ reads

$$
\dot{\vec{r}}^{\prime}=\dot{\vec{r}}-\dot{\vec{r}}_{0}=\vec{\omega} \times\left(\vec{r}-\vec{r}_{0}\right)=\vec{\omega} \times \vec{r}^{\prime},
$$

which is formally identical to the equation in $\mathcal{S}$, replacing $\vec{r}^{\prime}$ by $\vec{r}$. That is, in the frame $\mathcal{S}^{\prime}$, the fluid is seen to be rigidly rotating about the new origin $\vec{r}^{\prime}=0$ (or $\vec{r}=\vec{r}_{0}$, in the coordinates of $\mathcal{S}$ ). Since the cylinder is infinite, the picture in the frame $\mathcal{S}^{\prime}$ is indistinguishable from the one at $\mathcal{S}$. We see therefore that any point $\vec{r}$ rotating rigidly with angular velocity $\vec{\omega}$ in the frame $\mathcal{S}$ can be an axis of rotation for the fluid indistinguishable from the "original one".

Therefore, whilst the gravitomagnetic field and tidal tensor, as well as the mapping via KleinGordon equation in [1], link to the magnetic analogue of the Gödel universe, the gravitoelectric tidal tensor links to the Newtonian analogue, both yielding consistent models to picture the homogeneous rotation of this universe. 


\section{$5 \quad$ Linear gravitoelectromagnetism}

The oldest and best known gravito-electromagnetic analogies are the ones based on linearized gravity, which have been worked out by many authors throughout the years, see e.g. [7, 8, 14, 9, 10, 11, 12, 90, 16, 13, 64, 106. As is usually presented, one considers a metric given by small perturbations $\left|\varepsilon_{\alpha \beta}\right| \ll 1$ around Minkowski spacetime, $g_{\alpha \beta}=\eta_{\alpha \beta}+\varepsilon_{\alpha \beta}$, and from the components $\varepsilon_{\alpha \beta}$ one defines the 3 -vectors $\vec{G}$ and $\vec{H}$, in terms of which one writes the gravitational equations. Let us write the line element of such metric in the general form

$$
d s^{2}=-(1+2 \Phi) d t^{2}+2 \mathcal{A}_{j} d t d x^{j}+\left(\delta_{i j}+2 \xi_{i j}\right) d x^{i} d x^{j}
$$

If ones considers stationary perturbations, as is more usual (e.g. [7, 12, 90, 16, 13, 64, 106]), the GEM fields are (up to numerical factors in the different definitions) $\vec{G}=-\nabla \Phi, \vec{H}=\nabla \times \overrightarrow{\mathcal{A}}$, where, in this section (and only herein!), $\nabla_{i} \equiv \partial / \partial x_{i}$ (equaling the covariant derivative operator associated to the background Euclidean metric $\delta_{i j}$ ). These fields are straightforwardly related to the ones in Sec. 3. they are just, to linear order, minus the acceleration and twice the vorticity of the zero 3 -velocity observers $\left(u^{i}=0\right)$ with respect to the coordinate system used in 135) (they can be called "static observers"). Thus they are simply a linear approximation to the quasi-Maxwell fields in Eqs. 70.

If the fields depend on time, different definitions of the fields exist in the literature, as a complete, one to one GEM analogy based on inertial fields, holding simultaneously for the geodesic equation and for the field equations, is not possible, as we shall see below (cf. also [8, 9, 10, 2, 51]). So if one chooses to write one of them in an electromagnetic like form, the other will contain extra terms. We stick to defining $\vec{G}$ and $\vec{H}$ by minus the acceleration and twice the vorticity of the observer congruence (i.e. the same definitions given in Sec. 3.2 for congruence adapted frames, only this time linearized), which seems to make more sense from a physical point of view, as with these definitions the fields appear in the equation of geodesics playing roles formally analogous to the electric and magnetic fields in the Lorentz force. That amounts to define:

$$
\vec{G}=-\nabla \Phi-\frac{\partial \overrightarrow{\mathcal{A}}}{\partial t} ; \quad \vec{H}=\nabla \times \overrightarrow{\mathcal{A}}
$$

The space part of the linearized equation for the geodesics, in the coordinate basis $\mathbf{e}_{\alpha} \equiv \partial_{\alpha}$ associated to the coordinate system in (135), is obtained from the corresponding exact equation (52), for orthonormal tetrads, as follows 20 . One first notes that the coordinate triad of basis vectors $\mathbf{e}_{i}$ are connecting vectors between the $u^{i}=0$ observers; thus they co-rotate with the congruence, and therefore the orthonormal tetrad which follows $\mathbf{e}_{\alpha}$ as close as possible is the congruence adapted tetrad (obtained by setting $\vec{\Omega}=\vec{\omega}=\vec{H} / 2$, cf. Sec. 3.1); i.e., a tetrad such that $\mathbf{e}_{\hat{0}} \propto \mathbf{e}_{0}$ (for one to be dealing with the same observers) and that $\mathbf{e}_{\hat{i}}$ co-rotates with the $\mathbf{e}_{i}$, but without enduring the shear and expansion effects of the former (since the $\mathbf{e}_{\hat{\alpha}}$ remain orthonormal). Let $e_{\hat{\alpha}}^{\beta}$ denote the transformation matrix between $\mathbf{e}_{\alpha}$ and $\mathbf{e}_{\hat{\alpha}}: \mathbf{e}_{\hat{\alpha}}=e_{\hat{\alpha}}^{\beta} \mathbf{e}_{\beta}$. To linear order, $e_{\hat{\alpha}}^{\beta}$, and its inverse $e_{\alpha}^{\hat{\beta}}$,

\footnotetext{
${ }^{20}$ One could also obtain it directly from the covariant version $(64), 66$, by setting therein $\Omega^{\alpha}=\omega^{\alpha}=H^{\alpha} / 2$, noting that, to linear order $\Gamma_{0 k}^{i}=\epsilon^{i}{ }_{j k} H^{j} / 2+\partial \xi_{k}^{i} / \partial t$, and using 137 , as done below.
} 
are given by:

$$
\begin{array}{ll}
\mathbf{e}_{\hat{0}}=(1-\Phi) \mathbf{e}_{0} ; & \mathbf{e}_{\hat{i}}=\mathbf{e}_{i}-\xi_{i}{ }^{j} \mathbf{e}_{j}-\mathcal{A}_{i} \mathbf{e}_{0} ; \\
\mathbf{e}_{0}=(1+\Phi) \mathbf{e}_{\hat{0}} ; & \mathbf{e}_{i}=\mathbf{e}_{\hat{i}}+\xi_{i}{ }^{\hat{j}} \mathbf{e}_{\hat{j}}+\mathcal{A}_{i} \mathbf{e}_{\hat{0}} .
\end{array}
$$

Thus, $U^{\hat{i}}=e_{\alpha}^{\hat{i}} U^{\alpha}=U^{i}+\xi_{i}{ }^{j} U_{j}$; using $U^{i}=d x^{i} / d \tau$, substituting into 52, linearizing in the perturbations and keeping lowest order terms in $U^{i}$, and noting that, to linear order,

$$
K_{(i j)} \equiv u_{(i ; j)}=\sigma_{i j}+\frac{1}{3} \theta \delta_{i j} \approx \frac{\partial \xi_{i j}}{\partial t} ; \quad \theta=K_{i}^{i}=\frac{\partial \xi_{i}^{i}}{\partial t}
$$

the equation for the geodesics reads:

$$
\frac{d \vec{U}}{d t}=\vec{G}+\vec{U} \times \vec{H}-2 \frac{\partial \xi_{j}^{i}}{\partial t} U^{j} \vec{e}_{i}
$$

That is, the extra term, compared to the Lorentz force of electromagnetism, comes from the time derivative of the spatial metric (which is true also in the exact case, as we have seen in Sec. 3.2). Noting that $d \vec{U} / d t \approx d^{2} \vec{x} / d t^{2}-\vec{v} \partial \Phi / \partial t$, with $\vec{v}=d \vec{x} / d t$, we can also write this result as

$$
\frac{d^{2} \vec{x}}{d t^{2}}=\vec{G}+\vec{v} \times \vec{H}-2 \frac{\partial \xi_{j}^{i}}{\partial t} v^{j} \vec{e}_{i}+\frac{\partial \Phi}{\partial t} \vec{v} .
$$

The gravitational field equations in this regime are obtained by linearizing (99)-(104) and substituting relations 137 :

$$
\begin{array}{ccc}
\nabla \cdot \vec{G}=-4 \pi\left(2 \rho+T_{\alpha}^{\alpha}\right)-\frac{\partial^{2} \xi_{i}^{i}}{\partial t^{2}} ; & (\mathrm{i}) & \nabla \times \vec{G}=-\frac{\partial \vec{H}}{\partial t} \\
\nabla \cdot \vec{H}=0 ; \quad(\mathrm{iii}) & \nabla \times \vec{H}=-16 \pi \vec{J}+4 \frac{\partial}{\partial t} \xi_{j}{ }^{[j, k]} \vec{e}_{k} \\
G_{j, i}+\frac{1}{2} \epsilon_{i j k} \frac{\partial H^{k}}{\partial t}+\frac{\partial^{2}}{\partial t^{2}} \xi_{i j}+2 \xi_{(j, i) k}^{k}-\nabla^{2} \xi_{i j}-\xi_{k, i j}^{k}=8 \pi\left(T_{i j}+\frac{1}{2} \delta_{i j} T_{\alpha}^{\alpha}\right) .
\end{array}
$$

Eqs. (140), (140 v), and (140v), are, respectively, the time-time, time-space, and space-space components of Einstein's equations with sources (14); Eqs. (140 iii) and (140ii) are, respectively the time-time and space-time components of the identities $(14 \mathrm{~b})$. To obtain $(140 \mathrm{r})$ from the exact Eq. (101), we note that $\tilde{R}_{i j}$ reads, to linear order

$$
\tilde{R}_{i j} \simeq \Gamma_{i j, k}^{k}-\Gamma_{k j, i}^{k} \simeq 2 \xi_{(j, i) k}^{k}-\nabla^{2} \xi_{i j}-\xi_{k, i j}^{k}
$$

As for the time-space component of the identity (14b), i.e., Eq. 104), it yields the trivial, at linear order, equation $\star \tilde{R}_{j i}^{j}=0$.

Eqs. (140) encompass two particularly important regimes: the "GEM limit", and gravitational radiation. Starting by the latter, in a source free region $\left(T^{\alpha \beta}=0\right)$ one can, as is well known, through gauge transformations (employing the harmonic gauge condition, and further specializing to the transverse traceless, or radiation, gauge, see e.g. [12]) make $\overrightarrow{\mathcal{A}}=\Phi=\xi_{i}^{i}=\xi^{i j}{ }_{j}=0$; with this choice, the only non trivial equation left is $(140 \mathrm{v})$, yielding the $3-\mathrm{D}$ wave equation $\partial^{2} \xi_{i j} / \partial t^{2}=\nabla^{2} \xi_{i j}$.

The GEM regime is obtained making $\xi_{i j}=-\Phi \delta_{i j}$ (which effectively neglects radiation); in this case, the traceless shear of the congruence of zero 3 -velocity observers $\left(u^{i}=0\right.$ in the coordinates system of (135) vanishes, $\sigma_{\alpha \beta}=0$, and we have $u_{(i ; j)}=\theta \delta_{i j} / 3=-\delta_{i j} \partial \Phi / \partial t$. This is also the 
case for the post-Newtonian regime (e.g. [27, 62, 57, 97, 67]). Moreover, the source is assumed to be non-relativistic, so that the contribution of the pressure and stresses in Eq. (140) is negligible: $2 \rho+T_{\alpha}^{\alpha} \approx \rho$. The two versions of the equation for the geodesics, (138) and (139), then read, respectively,

$$
\frac{d \vec{U}}{d t}=\vec{G}+\vec{U} \times \vec{H}+2 \frac{\partial \Phi}{\partial t} \vec{v} ; \quad \frac{d^{2} \vec{x}}{d t^{2}}=\vec{G}+\vec{v} \times \vec{H}+3 \frac{\partial \Phi}{\partial t} \vec{v}
$$

and Eqs. 140 above become

$$
\begin{array}{r}
\nabla \cdot \vec{G}=-4 \pi \rho+3 \frac{\partial^{2} \Phi}{\partial t^{2}} ; \quad \text { (i) } \quad \nabla \times \vec{G}=-\frac{\partial \vec{H}}{\partial t} ; \\
\nabla \cdot \vec{H}=0 ; \quad(\text { iii }) \quad \begin{array}{r}
\quad \\
\frac{\partial}{\partial t} \mathcal{A}_{(i, j)}-\left(\frac{\partial^{2} \Phi}{\partial t^{2}}-\nabla^{2} \Phi\right) \delta_{i j}=-4 \pi \rho \delta_{i j} . \quad(\mathrm{v})
\end{array}
\end{array}
$$

In some works, e.g. [1], the gravitoelectric field is given a different definition: $\vec{G}^{\prime}=-\nabla \Phi-\frac{1}{4} \partial \overrightarrow{\mathcal{A}} / \partial t$. With this definition, and choosing the harmonic gauge condition, which implies $\nabla \cdot \overrightarrow{\mathcal{A}}=-4 \partial \Phi / \partial t$, the non-Maxwellian term in Eq. (142) disappears; but, on the other hand, a "non-Lorentzian" term appears in the equations for the geodesics, where in the place of $\vec{G}$ in Eqs. 138)-(139), we would have instead $\vec{G}^{\prime}-\frac{3}{4} \partial \overrightarrow{\mathcal{A}} / \partial t$. As for the non-Maxwellian term in Eq. $\left.142 \mathrm{v}\right)$, it is neglected in the post-Newtonian regime [62, 27].

The presence of the terms $\partial \vec{H} / \partial t$ and $\partial \vec{G} / \partial t$, "inducing" curls in $\vec{G}$ and $\vec{H}$, respectively, analogous to the induction terms of electromagnetism, leads to the question of whether one can talk about gravitational induction effects in analogy with electrodynamics. Indeed, there is a debate concerning the applicability and physical content of this analogy for time-dependent fields, see e.g. [1] and references therein. Although a discussion of the approaches to this issue in the literature is outside the scope of this work, still there are some points that can be made based on the material herein. If one considers a time dependent gravitational field, such as the one generated by a moving point mass, e.g. Eq. (2.10) of [51], one finds that indeed the corresponding gravitoelectric field $\vec{G}$ is different from the one of a point mass at rest, and has a curl. That is, the acceleration $-\vec{G}$ of the congruence of observers at rest with respect to the background inertial frame (the "post-Newtonian grid", e.g. [57]), acquires a curl when the source moves with respect to that frame. From Eq. (142ii), one can think about this curl as induced by the time-varying gravitomagnetic field $\vec{H}$, see e.g. [97]. These fields are well suited to describe the apparent Newtonian and Coriolis-like accelerations of particles in geodesic motion, as shown by Eq. 1141) above (one must just bear in mind that in the case of time-dependent fields, the motion is not determined solely by $\vec{G}$ and $\vec{H}$; there is an additional term with no analogue in the Lorentz force law, which leads to important differences). However, the latter are artifacts of the reference frame; the physical (i.e., tidal) forces tell a different story, as one does not obtain the correct tidal forces by differentiation of $\vec{G}$ and $\vec{H}$ (as is the case with electrodynamics). Namely, the curls of the GEM fields do not translate into these forces. The linearized gravitoelectric tidal tensor, Eq. 124$)$, reads in the GEM regime $\left(K_{(i j)}=-\delta_{i j} \partial \Phi / \partial t\right)$,

$$
\mathbb{E}_{i j} \approx-G_{i, j}+\frac{1}{2} \epsilon_{i j k} \frac{\partial H^{k}}{\partial t}-\frac{\partial \Phi}{\partial t} \delta_{i j}=-G_{(i, j)}-\frac{\partial \Phi}{\partial t} \delta_{i j}
$$

where we see that the curl 142 ii) is subtracted from the derivative of $\vec{G}$. That is, only the symmetrized derivative $G_{(i, j)}$ describes physical, covariant forces. This is manifest in the fact that the 
curl of $\vec{G}$ does not induce a rotation on a set of neighboring particles (the gravitational field only shears the set, see Sec. 2.2 and Eq. (26) therein), nor does it torque a rigid test body, see [6]. Note that in electromagnetism this rotation and torque are tidal manifestations of Faraday's law of induction. Likewise, the curl of $\vec{H}$ is not manifest in the gravitomagnetic tidal effects (e.g., the force on a gyroscope); the linearized gravitomagnetic tidal tensor $124 \mathrm{~b}$ ) reads, in this regime:

$$
\mathbb{H}_{i j} \approx-\frac{1}{2}\left[H_{i, j}-2 \epsilon_{i j l}\left(\frac{\partial G^{l}}{\partial t}-\frac{\partial^{2} \mathcal{A}^{l}}{\partial t^{2}}\right)\right],
$$

where again we can see that the induction contribution $4 \partial \vec{G} / \partial t$ (and also the one of the term $\partial^{2} \overrightarrow{\mathcal{A}} / \partial t^{2}$ ) to the curl of $\vec{H}$ is subtracted from the derivative of $\vec{H}$. The physical consequences are explored in [6]: in electromagnetism, due to vacuum equation $\nabla \times \vec{B}=\partial \vec{E} / \partial t$, there is a nonvanishing force on a magnetic dipole, $F_{E M}^{i}=B^{\beta i} \mu_{\beta}\left(=\nabla^{i}(\vec{\mu} \cdot \vec{B})\right.$ in the comoving inertial frame, cf. Eq. (125), whenever it moves in a non-homogeneous field; this is because the electric field measured by the particle is time-varying, and so $\nabla \times \vec{B} \neq 0 \Rightarrow B_{i j} \neq 0 \Rightarrow \vec{F}_{E M} \neq 0$. That is not necessarily the case in gravity. In vacuum, from Eqs. $(142 \mathrm{kv})$ and $(144)$, we have $\mathbb{H}_{i j}=-H_{(i, j)} / 2$, and the gravitational force on a gyroscope, cf. Eq. (1,2b) of Table 1 , is $F_{G}^{i}=\frac{1}{2} H^{(i, j)} S_{j}$. Thus no analogous induction effect is manifest in the force, and in fact spinning particles in non-homogeneous gravitational fields can move along geodesics, as exemplified in [6].

As for the equation of motion for the gyroscope's spin vector, from Eq. 27) we get, in terms of components in the coordinate system associated to 135 ,

$$
\frac{d S^{i}}{d t}=-\Gamma_{0 j}^{i} S^{j}=\frac{1}{2}(\vec{S} \times \vec{H})^{i}-\frac{\partial \Phi}{\partial t} S^{i}
$$

Comparing with the equation for the precession of a magnetic dipole (with respect to an inertial frame), $d \vec{S} / d \tau=\vec{\mu} \times \vec{B}$, there is a factor of $1 / 2$, and an additional term. The origin of the former is explained in Sec. 3.3 . it is due to the fact the field $\vec{H}$, causing the Coriolis (or gravitomagnetic) acceleration of test particles via Eq. (138), is distinct from the field causing the gyroscope precession in 145; in general they are independent. $\vec{H}$ is the sum of the vorticity $\vec{\omega}$ of the observer congruence with the angular velocity of rotation $\vec{\Omega}$ of the frame's spatial triads relative to Fermi-Walker transport; and to Eq. (145) only the latter part contributes. In the case of a congruence adapted frame $(\vec{\Omega}=\vec{\omega})$, which is the problem at hand (the frame is adapted to the congruence of $u^{i}=0$ observers), this originates the relative factor of $1 / 2$. Note also that the same factor shows up also in the force on the gyroscope discussed above, but in this case by the opposite reason: to $\vec{F}_{G}$ the vorticity $\vec{\omega}$ is the only part of $\vec{H}$ that contributes, cf. Eq. 117). The second term in 145 merely reflects the fact that the basis vectors $\mathbf{e}_{i}$ expand; if, using expressions (136), one transforms to the orthonormal basis $S^{i}=e^{i} S_{i}^{\hat{i}}$, and substitutes into 145 , that term vanishes, as expected from the exact result 72 .

If the field is stationary, we have a one to one correspondence with electromagnetism in inertial frames. Eq. (140 ) above becomes identical to (140), and then we are left with a set of four equations - Eqs. 140 - $140 \mathrm{v})$ with the time dependent terms dropped — similar, up to some factors, to the time-independent Maxwell equations in an inertial frame. These equations can also be obtained by linearization of Eqs. (24b)- $(24 \mathrm{~b})$ of Table 2. The space part of the equation of the geodesics: $d^{2} \vec{x} / d t^{2}=\vec{G}+\vec{v} \times \vec{H}$, cf. Eq. 139 above, is also similar to the Lorentz force in a Lorentz frame. The equation for the evolution of the spin vector of a gyroscope, in the coordinate 
basis, becomes simply $d \vec{S} / d \tau=\vec{S} \times \vec{H} / 2$, which gives the precession relative to the background Minkowski frame, and is similar to the precession of a magnetic dipole in a magnetic field. The force on a gyroscope whose center of mass it at rest is $\vec{F}_{G}=\nabla(\vec{S} \cdot \vec{H}) / 2$, similar to the force $\vec{F}_{E M}=\nabla(\vec{\mu} \cdot \vec{B})$ on a magnetic dipole at rest in a Lorentz frame; the same for the differential precession of gyroscopes/dipoles at rest: for a spatial separation vector $\delta x^{\alpha}$ they read, respectively, $\delta \vec{\Omega}_{G}=-\nabla(\delta \vec{x} \cdot \vec{H}) / 2$ and $\delta \vec{\Omega}_{E M}=-\nabla(\delta \vec{x} \cdot \vec{B})$.

\section{The formal analogy between gravitational tidal tensors and electromagnetic fields}

There is a set of analogies, based on exact expressions, relating the Maxwell tensor $F^{\alpha \beta}$ and the Weyl tensor $C_{\alpha \beta \gamma \delta}$. These analogies rest on the fact that: 1) they both irreducibly decompose into an electric and a magnetic type spatial tensors; 2) these tensors obey differential equations - Maxwell's equations and the so called "higher order" gravitational field equations - which are formally analogous to a certain extent [35, 36, 32, 37, 38, and 3) they form invariants in a similar fashion [31, 32, 39, 40]. In this section we will briefly review these analogies and clarify their physical content in the light of the previous approaches.

The Maxwell tensor splits, with respect to a unit time-like vector $u^{\alpha}$, into its electric, $E^{\alpha} \equiv$ $\left(E^{u}\right)^{\alpha}=F_{\beta}^{\alpha} u^{\beta}$, and magnetic, $B^{\alpha} \equiv\left(B^{u}\right)^{\alpha}=\star F^{\alpha}{ }_{\beta} u^{\beta}$, parts; i.e., the electric and magnetic fields as measured by the observers of 4 -velocity $u^{\alpha}$. These are spatial vectors: $E^{\alpha} u_{\alpha}=B^{\alpha} u_{\alpha}=0$, thus possessing $3+3$ independent components, which completely encode the 6 independent components of $F_{\mu \nu}$, as can be seen explicitly in the decompositions (1). In spite of their dependence on $u^{\alpha}$, one can use $E^{\alpha}$ and $B^{\beta}$ to define two tensorial quantities which are $u^{\alpha}$ independent, namely

$$
E^{\alpha} E_{\alpha}-B^{\alpha} B_{\alpha}=-\frac{F_{\alpha \beta} F^{\alpha \beta}}{2}, \quad E^{\alpha} B_{\alpha}=-\frac{\star F_{\alpha \beta} F^{\alpha \beta}}{4}
$$

these are the only algebraically independent invariants one can define from the Maxwell tensor.

The Weyl tensor has a formally similar decomposition: with respect to a unit time-like vector $u^{\alpha}$, it splits irreducibly into its electric, $\mathcal{E}_{\alpha \beta} \equiv\left(\mathcal{E}^{u}\right)_{\alpha \beta}=C_{\alpha \gamma \beta \sigma} u^{\gamma} u^{\sigma}$, and magnetic, $\mathcal{H}_{\alpha \beta} \equiv\left(\mathcal{H}^{u}\right)_{\alpha \beta}=$ $\star C_{\alpha \gamma \beta \sigma} u^{\gamma} u^{\sigma}$, parts. These two spatial tensors, both of which are symmetric and traceless (hence have 5 independent components each), completely encode the 10 independent components of the Weyl tensor, as can be seen by writing [35]

$$
C_{\alpha \beta}^{\gamma \delta}=4\left\{2 u_{[\alpha} u^{[\gamma}+g_{[\alpha}^{[\gamma}\right\} \mathcal{E}_{\beta]}^{\delta]}+2\left\{\epsilon_{\alpha \beta \mu \nu} u^{[\gamma} \mathcal{H}^{\delta] \mu} u^{\nu}+\epsilon^{\gamma \delta \mu \nu} u_{[\alpha} \mathcal{H}_{\beta] \mu} u_{\nu}\right\}
$$

(in vacuum, this equals decomposition (15)). Again, in spite of their dependence on $u^{\alpha}$, one can use $\mathcal{E}_{\alpha \beta}$ and $\mathcal{H}_{\alpha \beta}$ to define the two tensorial quantities which are $U^{\alpha}$ independent,

$$
\mathcal{E}^{\alpha \beta} \mathcal{E}_{\alpha \beta}-\mathcal{H}^{\alpha \beta} \mathcal{H}_{\alpha \beta}=\frac{C_{\alpha \beta \mu \nu} C^{\alpha \beta \mu \nu}}{8}, \quad \mathcal{E}^{\alpha \beta} \mathcal{H}_{\alpha \beta}=\frac{\star C_{\alpha \beta \mu \nu} C^{\alpha \beta \mu \nu}}{16},
$$

which are formally analogous to the electromagnetic scalar invariants (146). Note however that, by contrast with the latter, these are not the only independent scalar invariants one can construct from $C_{\alpha \beta \mu \nu}$; there are also two cubic invariants, see e.g. [32, 42, 29, 41, 74, 44]. 
As stated above, these tensors obey also differential equations which have some formal similarities with Maxwell's; such equations, dubbed the "higher order field equations", are obtained from the Bianchi identities $R_{\sigma \tau[\mu \nu ; \alpha]}=0$. These, together with the field equations $(14$ a), lead to:

$$
C_{\nu \sigma \tau ; \mu}^{\mu}=8 \pi\left(T_{\nu[\tau ; \sigma]}-\frac{1}{3} g_{\nu[\tau} T_{; \sigma]}\right)
$$

Expressing $C_{\alpha \beta \delta \gamma}$ in terms of $\mathcal{E}_{\alpha \beta}$ and $\mathcal{H}_{\alpha \beta}$ using (147), and taking time and space projections of (149) using the projectors (2), we obtain, assuming a perfect fluid, the set of equations

$$
\begin{gathered}
\tilde{\nabla}^{\mu} \mathcal{E}_{\nu \mu}=\frac{8 \pi}{3} \tilde{\nabla}_{\nu} \rho+3 \omega^{\mu} \mathcal{H}_{\nu \mu}+\epsilon_{\nu \alpha \beta} \sigma_{\gamma}^{\alpha} \mathcal{H}^{\beta \gamma} ; \\
\operatorname{curl} \mathcal{H}_{\mu \nu}=\nabla_{\mathbf{u}}^{\perp} \mathcal{E}_{\mu \nu}+\mathcal{E}_{\mu \nu} \theta-3 \sigma_{\tau\langle\mu} \mathcal{E}_{\nu\rangle}^{\tau}-\omega^{\tau} \epsilon_{\tau \rho(\mu} \mathcal{E}_{\nu)}{ }^{\rho}-2 a^{\rho} \epsilon_{\rho \tau(\mu} \mathcal{H}_{\nu)}^{\tau}+4 \pi(\rho+p) \sigma_{\mu \nu} ; \\
\tilde{\nabla}^{\mu} \mathcal{H}_{\nu \mu}=-8 \pi(\rho+p) \omega_{\nu}-3 \omega^{\mu} \mathcal{E}_{\nu \mu}-\epsilon_{\nu \alpha \beta} \sigma_{\gamma}^{\alpha} \mathcal{E}^{\beta \gamma} ; \\
\operatorname{curl}_{\mu \nu}=-\nabla_{\mathbf{u}}^{\perp} \mathcal{H}_{\mu \nu}-\mathcal{H}_{\mu \nu} \theta+3 \sigma_{\tau\langle\mu} \mathcal{H}_{\nu\rangle}^{\tau}+\omega^{\tau} \epsilon_{\tau \rho(\mu} \mathcal{H}_{\nu)}^{\rho}-2 a^{\rho} \epsilon_{\rho \tau(\mu} \mathcal{E}_{\nu)}^{\tau},
\end{gathered}
$$

where, following the definitions in [35, 36], $\epsilon_{\mu \nu \rho} \equiv \epsilon_{\mu \nu \rho \tau} u^{\tau}, \operatorname{curl} A_{\alpha \beta} \equiv \epsilon_{(\alpha}^{\mu \nu} A_{\beta) \nu ; \mu}$, and the index notation $\langle\mu \nu\rangle$ stands for the spatially projected, symmetric and trace free part of a rank two tensor:

$$
A_{\langle\mu \nu\rangle} \equiv h_{(\mu}^{\alpha} h_{\nu)}^{\beta} A_{\alpha \beta}-\frac{1}{3} h_{\mu \nu} h_{\alpha \beta} A^{\alpha \beta}
$$

with $h_{\beta}^{\alpha} \equiv\left(h^{u}\right)_{\beta}^{\alpha}$, cf. Eq. (2). $\nabla_{\mathbf{u}}^{\perp}$ and $\tilde{\nabla}$ (which in the equations above we could have written as well $\nabla^{\perp}$, for they are the same along the spatial directions) are the derivative operators whose action on a spatial vector is defined in Eqs. (58) and (61), respectively ${ }^{21}$. For a rank two spatial tensor $A^{\alpha \beta}$, we have $\nabla_{\mathbf{u}}^{\perp} A^{\alpha \beta}=h^{\alpha}{ }_{\mu} h^{\beta}{ }_{\nu} \nabla_{\mathbf{u}} A^{\mu \nu}$ and $\tilde{\nabla}_{\alpha} A^{\alpha \beta}=h^{\alpha}{ }_{\mu} h^{\beta}{ }_{\nu} \nabla_{\alpha} A^{\mu \nu}$. As before, the quantities $\theta \equiv u_{; \alpha}^{\alpha}, \sigma_{\mu \nu} \equiv h_{\mu}^{\alpha} h_{\nu}^{\beta} u_{\alpha ; \beta}-\theta h_{\mu \nu} / 3, \omega^{\alpha} \equiv \epsilon_{\beta \gamma}^{\alpha} u_{\gamma ; \beta} / 2$ and $a^{\alpha}$ are, respectively, the expansion, shear, vorticity and acceleration of the congruence of observers with 4 -velocity $u^{\alpha}$.

The analogous electromagnetic equations are the ones in Sec. 3.4.1, which we can re-write as

$$
\begin{gathered}
\tilde{\nabla}_{\mu} E^{\mu}=4 \pi \rho_{c}+2 \omega_{\mu} B^{\mu} ; \\
\epsilon^{\alpha \gamma \beta} B_{\beta ; \gamma}=\nabla_{\mathbf{u}}^{\perp} E^{\alpha}-\sigma_{\beta}^{\alpha} E^{\beta}+\frac{2}{3} \theta E^{\alpha}-\epsilon_{\beta \gamma}^{\alpha} \omega^{\beta} E^{\gamma}+\epsilon_{\beta \gamma}^{\alpha} B^{\beta} a^{\gamma}+4 \pi j^{\langle\alpha\rangle} ; \\
\tilde{\nabla}_{\mu} B^{\mu}=-2 \omega_{\mu} E^{\mu} ; \\
\epsilon^{\alpha \gamma \beta} E_{\beta ; \gamma}=-\nabla_{\mathbf{u}}^{\perp} B^{\alpha}+\sigma_{\beta}^{\alpha} B^{\beta}-\frac{2}{3} \theta B^{\alpha}+\epsilon_{\beta \gamma}^{\alpha} \omega^{\beta} B^{\gamma}+\epsilon^{\alpha \mu \sigma} E_{\mu} a_{\sigma} .
\end{gathered}
$$

Eqs. (152) and (154) follow from Eqs. (77) and (79), respectively, by noting that, for an arbitrary spatial vector $A^{\alpha}$,

$$
A_{; \beta}^{\beta}=\left(\top_{\beta}^{\gamma}+h_{\beta}^{\gamma}\right)\left(\top_{\lambda}^{\beta}+h_{\lambda}^{\beta}\right) A_{; \gamma}^{\lambda}=\left(\top_{\lambda}^{\gamma}+h_{\lambda}^{\gamma}\right) A_{; \gamma}^{\lambda}=A^{\beta} a_{\beta}+\tilde{\nabla}_{\alpha} A^{\alpha} .
$$

Eqs. (153) and (155) follow from Eqs. (81) and (84) by decomposing $K_{(\alpha \beta)}=\sigma_{\alpha \beta}+\theta h_{\alpha \beta} / 3$.

\footnotetext{
${ }^{21}$ To make contact with the notation in $[35,36]$, we note that the restriction to the spatial directions of both $\tilde{\nabla}$ and $\nabla^{\perp}$ yields the $3-\mathrm{D}$ connection " $\bar{\nabla}$ " of [36] (" $D$ " of [35]).
} 
It is worth mentioning that the exact wave equations for $E^{\alpha}$ and $B^{\alpha}$ in this formalism were obtained in [110], Eqs. (39)-(40) therein ${ }^{22}$, As for the exact wave equations for $\mathcal{E}_{\alpha \beta}$ and $\mathcal{H}_{\alpha \beta}$, they have not, to our knowledge, been derived in the literature; only in some approximations, such as in e.g. [38, 77, or the linear regime of the next section.

\subsection{Matte's equations vs Maxwell equations. Tidal tensor interpretation of gravitational radiation.}

Table 3: Formal analogy between Maxwell's equations (differential equations for electromagnetic fields) and Matte's equations (differential equations for gravitational tidal tensors)

\begin{tabular}{|c|c|c|c|}
\hline Electromagnetism & & Linearized Gravity & \\
\hline Maxwell's Equations & & Matte's Equations & \\
\hline$E_{, i}^{i}=0$ & $3.1 \mathrm{a})$ & $\mathbb{E}_{, i}^{i j}=0$ & $3.1 \mathrm{~b})$ \\
\hline$B_{, i}^{i}=0$ & $3.2 \mathrm{a})$ & $\mathbb{H}_{, i}^{i j}=0$ & $3.2 \mathrm{~b})$ \\
\hline$\epsilon^{i k l} E_{l, k}=-\frac{\partial B^{i}}{\partial t}$ & $3.3 a)$ & $\epsilon^{i k l} \mathbb{E}_{l, k}^{j}=-\frac{\partial \mathbb{H}^{i j}}{\partial t}$ & $3.3 \mathrm{~b})$ \\
\hline$\epsilon^{i k l} B_{l, k}=\frac{\partial E^{i}}{\partial t}$ & $(3,4 a)$ & $\epsilon^{i k l} \mathbb{H}_{l, k}^{j}=\frac{\partial \mathbb{E}^{i j}}{\partial t}$ & $(3,4 b)$ \\
\hline Wave equations & & Wave equations & \\
\hline$\left(\frac{\partial^{2}}{\partial t^{2}}-\partial^{k} \partial_{k}\right) E^{i}=0$ & $(3.5 a)$ & $\left(\frac{\partial^{2}}{\partial t^{2}}-\partial^{k} \partial_{k}\right) \mathbb{E}_{i j}=0$ & $3.5 b)$ \\
\hline$\left(\frac{\partial^{2}}{\partial t^{2}}-\partial^{k} \partial_{k}\right) B^{i}=0$ & (3.6a) & $\left(\frac{\partial^{2}}{\partial t^{2}}-\partial^{k} \partial_{k}\right) \mathbb{H}_{i j}=0$ & (3.6b) \\
\hline
\end{tabular}

In vacuum, the Bianchi identities become:

$$
R_{\sigma \tau[\mu \nu ; \alpha]}=0 ; \quad(a) \quad R_{\alpha \beta \gamma ; \mu}^{\mu}=0 \quad(b)
$$

(the second equation following from the first and from vacuum equation $R_{\mu \nu}=0$ ). The formal analogy with Eqs. (6), for $j^{\alpha}=0$, is now more clear [32]. In a nearly Lorentz frame where $u^{i}=0$, and to linear order in the metric potentials, Eqs. (150)-(151), for vacuum, become Eqs. (3.1b)(3.4b) of Table 3, which are formally similar to Maxwell's equations in a Lorentz frame (3.1a)(3.4a). The analogy in Eqs. (3.1)-(3.4) was first found by Matte [31, and further studied by some other authors [32, 34, 33. Taking curls of Eqs. (3.3a)-(3.4a) we obtain the wave equations for the electromagnetic fields; and taking curls of (3.3b)-(3.4b), we obtain gravitational waves as wave equations for gravitational tidal tensors.

\footnotetext{
${ }^{22}$ The wave equations in [110, 111] are obtained using also the Ricci identities $2 \nabla_{[\gamma} \nabla_{\beta]} X_{\alpha}=R_{\delta \alpha \beta \gamma} X^{\delta}$, which couple the electromagnetic fields to the curvature tensor; this coupling is shown to lead to amplification phenomena, suggested therein as a possible explanation for the observed (and unexplained) large-scale cosmic magnetic fields.
} 
Hence, to this degree of accuracy, vacuum gravitational waves can be cast as a pair of oscillatory tidal tensors $\mathbb{E}_{\alpha \beta}, \mathbb{H}_{\alpha \beta}$, propagating in space by mutually inducing each other, just like the pair of fields $E^{\alpha}, B^{\alpha}$, in the case of the electromagnetic waves. Also, just like $E^{\alpha}$ and $B^{\alpha}$ are equal in magnitude and mutually orthogonal for a purely radiative field, the same applies to the waves in (3.5b)-(3.6b) of Table 3. In the electromagnetic case this implies that the two invariants (146) vanish; likewise, the gravitational invariants (148) also vanish for a solution corresponding to pure gravitational radiation according to Bel's second criterion (cf. e.g. 44] p. 53) - a definition based on "super-energy", see below.

An interesting aspect of this formulation of gravitational radiation, contrasting with the more usual approaches in the literature, e.g. [12, 52, 17, 3] - which consist of equations for the propagation of gauge fields (the components of the metric tensor), having no local physical significance (only their second derivatives may be related to physically measurable quantities, see in this respect [68]) - is that Eqs. (3.5b)-(3.6b) are equations for the propagation of tensors of physical forces, with direct translation in physical effects: the relative acceleration of two neighboring test particles via geodesic deviation equation (1,1b) of Table 1, the force on a spinning test particle, via MathissonPapapetrou-Pirani Eq. (1.2b), or the relative precession of two nearby gyroscopes, via Eqs. (29)-(30).

It is instructive to note this contrast: whereas in electromagnetic radiation it is the vector fields that propagate, gravitational radiation is a purely tidal effect, i.e., traveling tidal tensors not subsidiary to any associated (electromagnetic-like, or Newtonian-like) vector field; it is well known that there are no vector waves in gravity (see e.g. [63, 52, 10]; such waves would carry negative energy if they were to exist, cf. [52] p. 179). We have seen in Sec. 3.5 that, except for the very special case of the linear regime in weak, stationary fields (and static observers therein), the gravitational tidal tensors cannot be cast as derivatives of some vector field. In the electromagnetic case there are of course also tidal effects associated to the wave; but their dynamics follows trivially 23 from Eqs. (3.3a)-(3.4a) of Table 3 , to this accuracy, the tidal tensors as measured by the background static observers are just $E_{i j}=E_{i, j}, B_{i j}=B_{i, j}$; hence the equations of their evolution (i.e., the "electromagnetic higher order equations") are:

$$
\begin{gathered}
\epsilon_{i}{ }^{k l} E_{j l, k}=0 ; \quad \epsilon_{i}{ }^{k l} B_{j l, k}=0 \\
\epsilon_{i}{ }^{k l} E_{l j, k}=\epsilon_{i}{ }^{k l} E_{l k, j}=-\frac{\partial B_{i j}}{\partial t} ; \quad \epsilon_{i}{ }^{k l} B_{l j, k}=\epsilon_{i}{ }^{k l} B_{l k, j}=\frac{\partial E_{i j}}{\partial t} .
\end{gathered}
$$

These four equations are the physical analogues of the pair of gravitational Eqs. (3.3b)-(3.4b); we have two more equations in electromagnetism, since $E_{i j}$ and $B_{i j}$ are not symmetric. Eqs. (157), and the first equality in Eqs. (158), come from the fact that derivatives in flat spacetime commute; therefore $\epsilon_{i}{ }^{k l} E_{j l, k}=\epsilon_{i}{ }^{k l} E_{j,[l k]}=0$ and $E_{l j, k}=E_{l, j k}=E_{l k, j}$. Thus, Eqs. (158), which are the only ones that contain dynamics, are obtained by simply differentiating Eqs. (3.3a)-(3.4a) with respect to $x^{j}$. The wave equations for the electromagnetic tidal tensors follow likewise from differentiating Eqs. (3.5a)-(3.6a) with respect to $x^{j}$. Note that the fact that, in gravity, $\mathbb{H}_{j[l, k]} \neq 0$, is again related to the fact that, even in the linear regime, the gravitational tidal tensors are not derivatives of some vector fields.

\footnotetext{
${ }^{23}$ We thank J. Penedones for discussions on this point.
} 


\subsubsection{Super-energy}

Using the analogies herein as a guiding principle, a gravitational 4-index tensor $T^{\alpha \beta \gamma \delta}$ — the BelRobinson, or "super-energy" tensor, see e.g. [69, 35] - constructed from the curvature tensor in a way formally analogous to the way the energy-momentum tensor of the electromagnetic field $\left(T_{\mathrm{EM}}^{\alpha \beta}\right)$ is constructed from $F^{\alpha \beta}$, has been proposed. The motivation is to find local, covariant, quantities alternative to the gravitational energy and momentum given by the Landau-Lifshitz pseudo-tensor [18] (which can only have a meaning in a global sense, and in asymptotically flat spacetimes); the former however do not have the same dimensions, which has been posing difficulties in their physical interpretation. For a discussion on this issue and on the possible relation between energy and super-energy (which is still an open problem) we refer to [69] p. 31 (and references therein), and [68, 71, 72, 70, 73, 43, 91]. Herein we would just like to point out that the viewpoint that gravitational waves are characterized by a flow of super-energy fits well with their interpretation as a pair of propagating tidal tensors, since, as can be seen comparing Eqs. (23)-(24) to (40)-(41) of [35], the "super-energy" scalar $W \equiv T^{\alpha \beta \gamma \delta} u_{\alpha} u_{\beta} u_{\gamma} u_{\delta}$ and the "super-Poynting" vector $\mathcal{P}^{\langle\alpha\rangle} \equiv$ $-T^{\langle\alpha\rangle \beta \gamma \delta} u_{\beta} u_{\gamma} u_{\delta}$ (as measured by some observer $u^{\alpha}$ ), when written explicitly in terms of tidal tensors, are formally analogous to electromagnetic field energy $\rho_{E M} \equiv T_{E M}^{\alpha \beta} u_{\alpha} u_{\beta}$ and Poynting vector $p_{E M}^{\langle\alpha\rangle} \equiv-T_{E M}^{\langle\alpha\rangle} u_{\beta}$, only with $\mathbb{E}_{\alpha \beta}, \mathbb{H}_{\alpha \beta}$ in the place of the electromagnetic fields $E^{\alpha}, B^{\alpha}$.

\subsection{The relationship with the other GEM analogies}

The analogy drawn in this section is between the electromagnetic fields and the electric and magnetic parts of the Weyl tensor: $\left\{E^{\alpha}, B^{\alpha}\right\} \leftrightarrow\left\{\mathcal{E}_{\mu \nu}, \mathcal{H}_{\mu \nu}\right\}$. It is clear, from the discussion of the physical meaning of $\left\{\mathbb{E}_{\mu \nu}, \mathbb{H}_{\mu \nu}\right\}$ in Sec. 2 , and from the discussion in Sec. 3 of the dynamical gravitational counterparts of $\left\{E^{\alpha}, B^{\alpha}\right\}$, that this analogy is a purely formal one. It draws a parallelism between electromagnetic fields (whose dynamical gravitational analogues are the GEM inertial fields $\{\vec{G}, \vec{H}\}$ of Sec. 3), with gravitational tidal fields, which, as shown in Sec. 2, are the physical analogues not of $\left\{E^{\alpha}, B^{\alpha}\right\}$, but instead of the electromagnetic tidal tensors $\left\{E_{\mu \nu}, B_{\mu \nu}\right\}$ (these, in an inertial frame, are derivatives of the $E^{\alpha}$ and $B^{\alpha}$, cf. Eqs. (108)-(111)). This sheds light on some conceptual difficulties in the literature regarding the physical content of the analogy and in particular the physical interpretation of the tensor $\mathcal{H}_{\mu \nu}$, see [2] for details. It is also of crucial importance for the correct understanding of physical meaning of the curvature invariants, and their implications on the motion of test particles, which will be subject of detailed study elsewhere [29].

\section{When can gravity be similar to electromagnetism?}

The gravitational and electromagnetic interactions have many intrinsic differences, perhaps the most basic of them being that the equivalence principle between inertial mass and gravitational mass/charge has no counterpart in electrodynamics (in the multipole language of Sec. 2, no covariant gravitational force is exerted on a monopole particle, by contrast with the electromagnetic Lorentz force). But other important differences exist and are manifest in the approaches herein; at the same time striking similarities emerged.

A crucial point to realize is that the two exact physical gravito-electromagnetic analogies the tidal tensor analogy of Sec. 2, and the inertial GEM fields analogy of Sec. 3 - do not rely 
on a close physical similarity between the interactions; the gravitational objects $\left\{\vec{G}, \vec{H}, \mathbb{E}_{\alpha \beta}, \mathbb{H}_{\alpha \beta}\right\}$, despite playing analogous dynamical roles to the ones played by the objects $\left\{\vec{E}, \vec{B}, E_{\alpha \beta}, B_{\alpha \beta}\right\}$ in electromagnetism, are themselves in general very different from the latter, even for seemingly analogous setups (e.g. the EM field of spinning charge, and the gravitational field of a spinning mass). In this sense, these analogies have a different status compared to the popular GEM analogy based on linearized theory, which, in order to hold, require a degree of similarity between the interactions to which the former two are not bound.

What the tidal tensor formalism of Sec. 2 , together with the inertial fields formalism of Sec. 3 , provide, is a "set of tools" to determine under which precise conditions a similarity between the gravitational and electromagnetic interactions may be expected.

The key differences between electromagnetic and gravitational tidal tensors are: a) they do not exhibit, generically, the same symmetries; b) gravitational tidal tensors are spatial whereas the electromagnetic ones are not; c) electromagnetic tidal tensors are linear in the corresponding fields, whereas the gravitational ones are not.

The electromagnetic tidal tensors, for a given observer, only have the same symmetries and time-projections as the gravitational ones when the Maxwell tensor is covariantly constant along the observer's worldline; that is implied by Eqs. (1.8) and (1.5) of Table 1. This restricts the eligible setups to intrinsically stationary fields (i.e., whose time-dependence, if it exists, can be gauged away by a change of frame), and to a special class of observers therein; for electromagnetic fields in flat spacetime, those observers must be static in the inertial frame where the fields are explicitly timeindependent. This is an important point that is worth discussing in some detail. Consider the two basic analogous fields, the Coulomb field of a point charge, and the Schwarzschild gravitational field. Consider also in the latter observers $\mathcal{O}$ in circular motion: 4-velocity $U^{\alpha}=\left(U^{0}, 0,0, U^{\phi}\right)$, angular velocity $\Omega=U^{\phi} / U^{0}$. The worldlines of these observers are tangent to Killing vector fields: $U^{\alpha} \| \xi^{\alpha} ; \mathcal{L}_{\xi} g_{\alpha \beta}=0$. One can say (e.g. [52, 65]) that they see a constant spacetime geometry; for this reason they are called "stationary observers". Now consider observers in circular motion around a Coulomb charge. Despite moving along worldlines tangent to vector fields which are symmetries of the electromagnetic field: $U^{\alpha} \| \xi^{\alpha} ; \mathcal{L}_{\xi} F_{\alpha \beta}=0$, the observers $U^{\alpha}$ do not see a covariantly constant field: $F_{\alpha \beta ; \gamma} U^{\gamma} \neq 0$, which by virtue of Eqs. (1.5a), (1.8a), implies that the electromagnetic tidal tensors have an antisymmetric part (in particular the spatial part $B_{[i j]} \neq 0$ ), and thus means that they cannot be similar to their gravitational counterparts. This is a natural consequence of Maxwell's equations, and can be easily understood as follows. The magnetic tidal tensor measured by $\mathcal{O}$ is a covariant derivative of the magnetic field as measured in the inertial frame momentarily comoving with it: $B_{\alpha \beta} \equiv \star F_{\alpha \gamma ; \beta} U^{\gamma}=\left.B_{\alpha ; \beta}\right|_{U=\text { const }}=\left(B_{M C R F}\right)_{\alpha ; \beta}$. Now, $B_{[i j]} \neq 0$ means that $\vec{B}_{M C R F}$ has a curl; which is to be expected, since in the MCRF the electric field is time-dependent (constant in magnitude but varying in direction), which, by virtue of Maxwell's equation $\nabla \times \vec{B}=\partial \vec{E} / \partial t=\gamma \vec{E} \times \vec{\Omega}$ (holding in the MCRF, and for which 1, 1 a) is a covariant form) induces a curl in $\vec{B}$.

Even if one considers static observers in stationary fields, so that the gravitational and electromagnetic tidal tensors have the same symmetries, still one may not see a close similarity between the interactions. The electromagnetic tidal tensors are linear in the electromagnetic fields, and the latter themselves linear in the electromagnetic 4-potential $A^{\alpha}=(\phi, \vec{A})$, whereas the gravitational tidal tensors are non-linear in the GEM fields, as shown by Eqs. (116)-(117), the gravitomag- 
netic field $\vec{H}$ being itself non-linear in the metric potentials $\Phi, \overrightarrow{\mathcal{A}}$. This means that one can expect a similarity between tidal tensors in two limiting cases - linearized theory, and the ultrastationary spacetimes considered in Sec. 4, where $\Phi=\vec{G}=0$, and, therefore, cf. Eqs. (117) and (70), the exact gravitomagnetic tidal tensor is linear (both in the metric and in the GEM fields): $\mathbb{H}_{\hat{i} \hat{j}}=-\tilde{\nabla}_{\hat{j}} H_{\hat{i}} / 2=-\tilde{\nabla}_{\hat{j}}(\tilde{\nabla} \times \overrightarrow{\mathcal{A}})_{\hat{i}} / 2$. We have seen in Sec. 4 that there is indeed an exact mapping (via the Klein-Gordon equation) between the dynamics in these spacetimes and an electromagnetic setup.

In what concerns concrete effects, the precise conditions (namely regarding the time dependence of the fields) for occurrence of a gravito-electromagnetic similarity are specific to the type of effect. For the tidal effects (which imply physical, covariant gravitational forces) such as the force on a spinning particle or the worldline deviation of two neighboring particles, it is the tidal tensors as measured by the test particles (4-velocity $U^{\alpha}$ ) that determine the effects, cf. Eqs. (1.1)-(1.2); which means that it is along the particle's worldline that the constancy of the fields is required. This basically implies that the similarity only occurs at the instant when the particles are at rest in stationary fields, so it does not hold in a dynamical situation. In the case of the correspondence between the Lorentz force, Eq. (54), and the geodesic equation formulated as an inertial force (which is a reference frame effect), we see from Eq. (52) that the requirement is that the frame is rigid, i.e. $\sigma_{\alpha \beta}=\theta=0$; as explained in Sec. 3.2. this amounts to saying that the spatial part of the metric (in the coordinates associated to such frame) must be time-independent. This can also be stated in the following manner, generalizing to the exact case the conclusion obtained in [51] in the context of the post-Newtonian approximation: in the case of the GEM analogy for the geodesic equation, the stationarity of the fields is required in the observer's frame (not in the test particle's frame! The test particles can move along arbitrary worldlines). As for the gyroscope "precession" (72) and the correspondence with the precession of a magnetic dipole (73), there is no restriction on the time dependence of the fields.

\section{Conclusion}

In this work we collected and further developed different gravito-electromagnetic analogies existing in the literature, and clarified the connection between them. A detailed summary of the material in this paper is given in the introduction; herein we conclude by briefly summarizing the main outcome of each approach, and their applicability. The analogies split into two classes: physical and purely formal. In the second category falls the analogy between the electric and magnetic parts of the Weyl and Maxwell tensors, discussed in Sec 6. The physical analogies are divided into two classes: exact analogies, and the best known post-Newtonian and linearized theory approaches. Exact physical analogies are the analogy between the electromagnetic fields and the inertial fields of Sec. 3, and the tidal tensor analogy of Sec. 2 .

These analogies are useful from a practical point of view, as they provide a familiar formalism and insight from electromagnetic phenomena to describe otherwise more complicated gravitational problems. Indeed, there is a number of fundamental equations, summarized in Table 4, which can be obtained from the electromagnetic counterparts by simple application of the analogy. But the existence of these analogies, especially the exact, physical ones, is also interesting from the theoretical point of view, unveiling intriguing similarities — both in the tidal tensor, and in the 
inertial field formalism, manifest in Tables 1 and 2 , respectively — and enlightening differences.

The tidal tensor formalism is primarily suited for a transparent comparison between the two interactions, since it is based on mathematical objects describing covariant physical forces common to both theories. Comparing the tidal tensors of both sides is straightforward from Eqs. (1.3)-(1.7) of Table 1. Fundamental differences are encoded in their symmetries and time projections; herein we explored them in terms of the worldline deviation of (monopole) test particles; and in the companion paper [6], in terms of the dynamics of spinning multipole test particles. The latter is perhaps the most natural application of the formalism; but it can be useful in many other applications, namely gravitational radiation (as discussed in Sec. 6.1), and whenever one wishes to study the physical aspects of spacetime curvature.

Table 4: What can be computed by direct application of the GEM analogies

\begin{tabular}{|c|c|}
\hline Result & Approach \\
\hline $\begin{array}{l}\text { - Geodesic deviation equation }(1,1 \mathrm{~b}) \text { of Table } 1 \text {. } \\
\text {-Replacing }\left\{q, E_{\alpha \beta}\right\} \rightarrow\left\{m,-\mathbb{E}_{\alpha \beta}\right\} \text { in }(1,1 \mathrm{a}) \text {. } \\
\text { - Force on a gyroscope }(1,1 \mathrm{~b}): \\
\text {-Replacing }\left\{\mu^{\alpha}, B_{\alpha \beta}\right\} \rightarrow\left\{S^{\alpha},-\mathbb{H}_{\alpha \beta}\right\} \text { in }(1,1 \mathrm{a}) \text {. } \\
\text { - Gravitational field equations }(1,3 \mathrm{~b})-(1,4 \mathrm{~b}),(1,6 \mathrm{~b})-(1,7 \mathrm{~b}) \text { : } \\
\text {-Replacing }\left\{E_{\alpha \beta}, B_{\alpha \beta}\right\} \rightarrow\left\{\mathbb{E}_{\alpha \beta}, \mathbb{H}_{\alpha \beta}\right\} \text { in Eqs. (9)-(12), } \\
\quad \text { and } \rho_{c} \rightarrow 2 \rho+T_{\alpha}^{\alpha} \text { in }(9), j^{\alpha} \rightarrow 2 J^{\alpha} \text { in }(12) \text {. }\end{array}$ & $\begin{array}{l}\text { Tidal tensor analogy } \\
\text { (Exact, general results) }\end{array}$ \\
\hline $\begin{array}{l}\text { - Geodesic Equation }(68) \text { (stationary fields) } \\
\text {-Replacing }\{q, \vec{E}, \vec{B}\} \rightarrow\{m, \vec{G}, \vec{H}\} \text { in }[54) \text {, multiplying by } \gamma \text {. } \\
\text { - Gyroscope "precession" Eq. }(72\} \text { (arbitrary fields): } \\
\text {-Replacing }\{\vec{\mu}, \vec{B}\} \rightarrow\{\vec{S}, \vec{H} / 2\} \text { in }(73) \text {. } \\
\text { - Force on gyroscope Eq. } 127\} \text { (stationary fields, } \\
\text { particle's worldline tangent to time-like Killing vector): } \\
\text {-Replacing }\{\vec{\mu}, \vec{E}, \vec{B}\} \rightarrow\{\vec{S}, \vec{G}, \vec{H} / 2\} \text { in } 125 \text {, factor } \\
\text { of } 2 \text { in the last term. }\end{array}$ & $\begin{array}{l}\text { Inertial "GEM fields" analogy } \\
\text { (Exact results, require special frames) }\end{array}$ \\
\hline $\begin{array}{l}\text { - Higher order field equations }(3,1 \mathrm{~b})-(3,4 \mathrm{~b}) \text { : } \\
\text {-Replacing }\{\vec{E}, \vec{B}\} \rightarrow\left\{\mathbb{E}_{i j}, \mathbb{H}_{i j}\right\} \text { in Eqs. }(3,1 \mathrm{a})-(3,4 \mathrm{a}) \text {. } \\
\text { - Equations of gravitational waves }(3,5 \mathrm{~b})-(3.6 \mathrm{~b}) \text { : } \\
\text {-Replacing }\{\vec{E}, \vec{B}\} \rightarrow\left\{\mathbb{E}_{i j}, \mathbb{H}_{i j}\right\} \text { in Eqs. }(3,5 \mathrm{a})-(3,6 \mathrm{a}) \text {. }\end{array}$ & $\begin{array}{l}\text { Weyl-Maxwell tensors analogy } \\
\text { (Results for linearized theory) }\end{array}$ \\
\hline
\end{tabular}

The analogy based on inertial GEM fields from the $1+3$ formalism, Sec. 3, is a very powerful formalism, with vast applications; especially in the case of stationary spacetimes, where for arbitrarily strong fields the equation for geodesics is cast in a form similar to Lorentz force; many other 
effects related to frame-dragging can be treated exactly with the GEM fields: gyroscope "precession" [6, 27, 28, 57, 20], the Sagnac effect [93], the Faraday rotation [24], the force on a gyroscope (Sec. 3.6 and [20]; note however that it is not as general as the tidal tensor formulation of the same force); and other applications, such as the matching of stationary solutions [22], or describing the "hidden momentum" of spinning particles [6]. The general formulation of GEM fields in Sec. 3 . applying to arbitrary fields and frames, extends the realm of applicability of this formalism.

The well known analogies between electromagnetism and post-Newtonian and linearized gravity, follow as a limiting case of the exact approach in Sec. 3. In the case of the tidal effects, they can be seen also as a limiting case of the tidal tensor analogy of Sec. 2 (in the sense that for weak, time-independent fields, the gravitational tidal tensors reduce to derivatives of the GEM fields). Realizing this, and understanding the conditions under which linear GEM is obtained from the rigorous, exact approaches, is important for a correct interpretation of the physical meaning of the quantities involved, which is not clear in the usual derivations in the literature (this is especially the case for many works on linear GEM), and thus prone to misconceptions (see in this respect [2, 1]. On the other hand, linear GEM is the most important in the context of experimental physics, as it pertains all gravitomagnetic effects detected to date [58, 90, 59, 97, 96, 95], and the ones we hope to detect in the near future [60].

As for the analogy between the electric and magnetic parts of the Weyl and Maxwell tensors, its most important application is gravitational radiation, where it provides equations for the propagation of tensors of physical forces (not components of the metric tensor, as in the more usual approaches, which are pure gauge fields), with direct translation into physical effects (via the tidal tensor formalism of Sec. 2). This analogy has been used to address the fundamental questions of the content of gravitational waves, and the "energy" of the gravitational field. Namely, to propose covariant, local local quantities for the gravitational field analogous to the electromagnetic field energy and momentum densities - the "super-energy" and "super-momentum" densities encoded in the Bel tensor. The existing criteria for radiative states [92], states of intrinsic radiation [32, 91] or pure radiation (94, see also [44] p. 53), are also solely driven by this analogy. It is also useful for the understanding of the quadratic invariants of the curvature tensor; indeed, it will be shown elsewhere 29] that using the two approaches together - the formal analogies of Sec. 6 to gain insight into the invariant structure, and the tidal tensor analogy as a physical guiding principle one can explain, in the astrophysical applications of current experimental interest, the significance of the curvature invariants and the implications on the motion of test particles.

\section{Acknowledgments}

We thank J. Penedones, the anonymous referees and A. Editor for useful comments and remarks; we also thank A. García-Parrado and J. M. M. Senovilla for correspondence and useful discussions.

\section{A Inertial Forces - simple examples in flat spacetime}

In Sec. 3.2 we have seen that the inertial forces felt in a given frame arise from two independent contributions of different origin: the kinematics of the observer congruence (that is, from the derivatives of the temporal basis vector of the frame, $\mathbf{e}_{\hat{0}}=\mathbf{u}$, where $\mathbf{u}$ is the observers' 4 -velocity), 


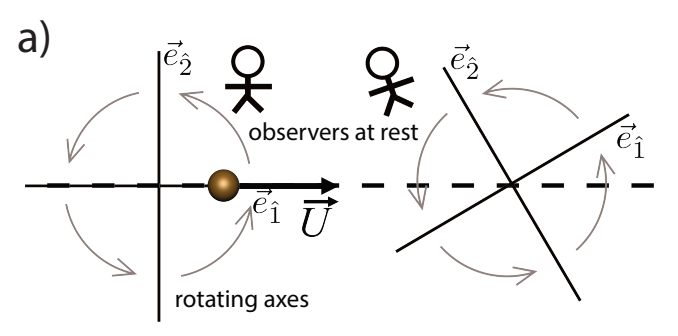

In the tetrad:
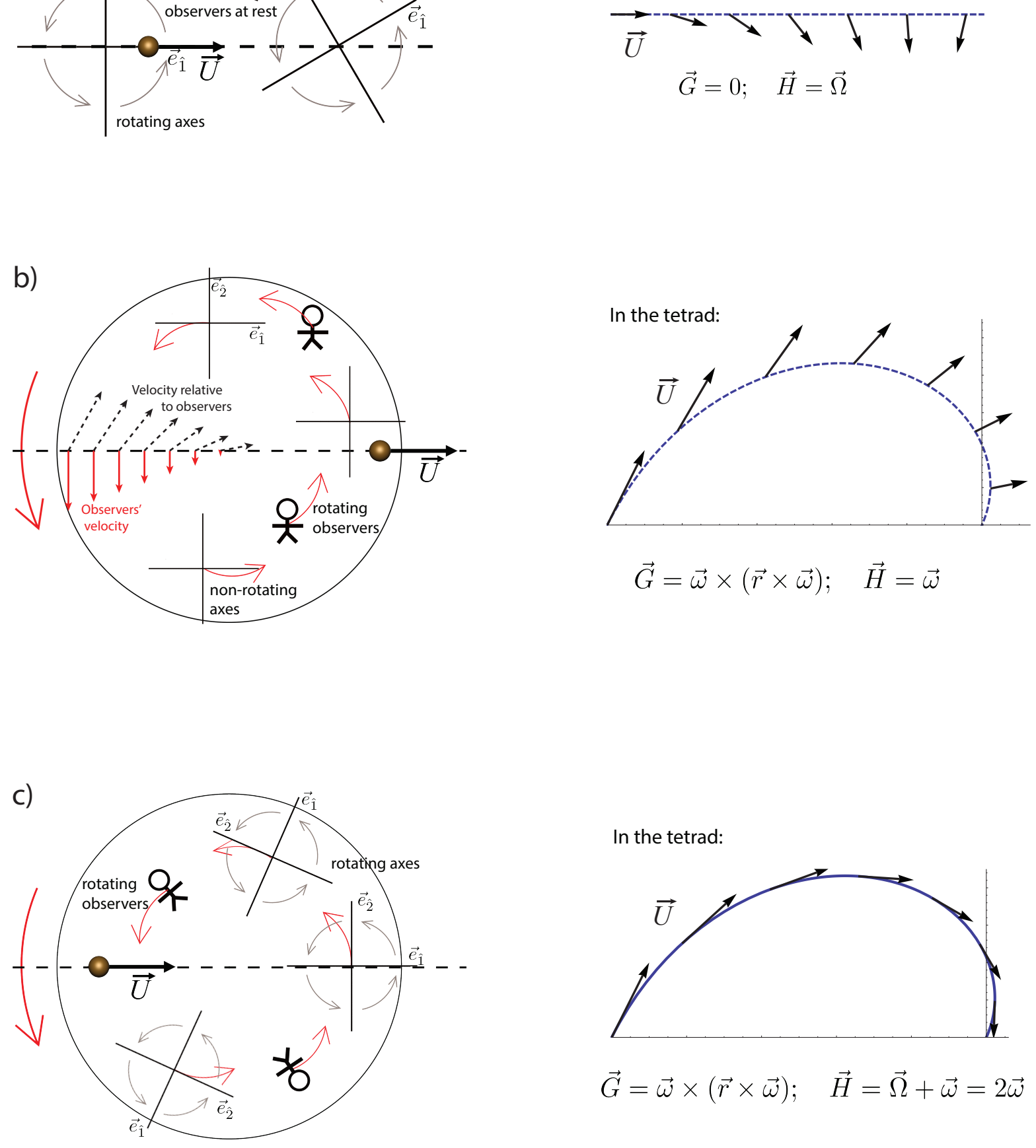

Figure 2: A test particle in uniform motion in flat spacetime from the point of view of three different frames: a) a frame composed of observers at rest, but carrying spatial triads that rotate with uniform angular velocity $\vec{\Omega}$; b) a frame consisting of a congruence of rigidly rotating observers (vorticity $\vec{\omega}$ ), but each of them carrying a non-rotating spatial triad (i.e., that undergoes FermiWalker transport); c) a rigidly rotating frame (a frame adapted to a congruence of rigidly rotating observers); the spatial triads co-rotate with the congruence, $\vec{\Omega}=\vec{\omega}$. Note: by observer's rotation we mean their circular motion around the center; and by axes rotation we mean their rotation (relative to FW transport) about the local tetrad's origin. 
and the transport law for the spatial triads $\mathbf{e}_{\hat{i}}$ along the congruence. In order to illustrate these concepts with simple examples, we shall consider, in flat spacetime, the straightline geodesic motion of a free test particle (4-velocity $\mathbf{U}$ ), from the point of view of three distinct frames: a) a frame whose time axis is the 4 -velocity of a congruence of observers at rest, but whose spatial triads rotate uniformly with angular velocity $\vec{\Omega}$; b) a frame composed of a congruence of rigidly rotating observers (vorticity $\vec{\omega}$ ), but carrying Fermi-Walker transported spatial triads $(\vec{\Omega}=0$ ); c) a rigidly rotating frame, that is, a frame composed of a congruence of rigidly rotating observers, carrying spatial triads co-rotating with the congruence $\vec{\Omega}=\vec{\omega}$ (i.e., "adapted" to the congruence, see Sec. 3.1). This is depicted in Fig. 2 ,

In the first case there we have a vanishing gravitoelectric field $\vec{G}=0$, and a gravitomagnetic field $\vec{H}=\vec{\Omega}$ arising solely from the rotation (with respect to Fermi-Walker transport) of the spatial triads; thus the only inertial force present is the gravitomagnetic force $\vec{F}_{\mathrm{GEM}}=\gamma \vec{U} \times \vec{\Omega}$, cf. Eq. (52), with $\gamma \equiv-U^{\alpha} u_{\alpha}$. In the frame b), there is a gravitoelectric field $\vec{G}=\vec{\omega} \times(\vec{r} \times \vec{\omega})$ due the observers acceleration, and also a gravitomagnetic field $\vec{H}=\vec{\omega}$, which originates solely from the vorticity of the observer congruence. That is, there is a gravitomagnetic force $\gamma \vec{U} \times \vec{\omega}$ which reflects the fact that the relative velocity $v^{\alpha}=U^{\alpha} / \gamma-u^{\alpha}$ (or $\vec{v}=\vec{U} / \gamma$, in the observer's frame, where $\vec{u}=0$ ) between the test particle and the observer it is passing by changes in time. The total inertial forces are in this frame

$$
\vec{F}_{\mathrm{GEM}}=\gamma[\gamma \vec{\omega} \times(\vec{r} \times \vec{\omega})+\vec{U} \times \vec{\omega}] .
$$

In the frame c), which is the relativistic version of the classical rigid rotating frame, one has the effects of a) and b) combined: a gravitoelectric field $\vec{G}=\vec{\omega} \times(\vec{r} \times \vec{\omega})$, plus a gravitomagnetic field $\vec{H}=\vec{\omega}+\vec{\Omega}=2 \vec{\omega}$, the latter leading to the gravitomagnetic force $2 \gamma \vec{U} \times \vec{\omega}$, which is the relativistic version of the well known Coriolis acceleration, e.g. [114]. The total inertial force is in this frame

$$
\vec{F}_{\mathrm{GEM}}=\gamma[\gamma \vec{\omega} \times(\vec{r} \times \vec{\omega})+2 \vec{U} \times \vec{\omega}]
$$

which is the relativistic generalization of the inertial force in e.g. Eq. (4.91) of [114]. Moreover, in this case, as discussed in Secs. 3.2.1 and 3.2.2, the $\Gamma_{\hat{j} \hat{k}}^{\hat{i}}$ in Eq. 53 are the connection coefficients of the (Levi-Civita) 3-D covariant derivative with respect to the metric $h_{i j}$ (defined by Eq. (69p) defined on the space manifold associated to the quotient of the spacetime by the congruence; $\vec{U}$ is the vector tangent to the 3-D curve (see Fig. 22) obtained by projecting the particle's worldline on the space manifold, and $\vec{F}_{\mathrm{GEM}}=\tilde{D} \vec{U} / d \tau$ is simply the covariant $3-\mathrm{D}$ acceleration of that curve.

\section{References}

[1] L. F. Costa, C. A. R. Herdeiro, Phys. Rev. D 78, 024021 (2008).

[2] L. F. Costa, C. A. R. Herdeiro, [arXiv:gr-qc/0612140].

[3] C. Corda, Phys. Rev. D 83, 062002 (2011).

[4] D. Nichols et al., Phys. Rev. D 84, 124014 (2011).

[5] L. Bel, C. R. Acad. Sci. Paris 246, 3015 (1958). 
[6] L. F. Costa, J. Natário, M. Zilhão, [arXiv:1207.0470].

[7] I. Ciufolini, J. A. Wheeler, Gravitation and Inertia, Princeton Series in Physics (Princeton University, Princeton, NJ, 1995).

[8] H. C. Ohanian, R. Ruffini, Gravitation and Spacetime (W.W. Norton, New York and London, 1994), 2nd ed.

[9] E. G. Harris, Am. J. Phys. 59, 421 (1991).

[10] S. J. Clark, R. W. Tucker, Class. Quant. Grav. 17, 4125 (2000).

[11] M. L. Ruggiero, A. Tartaglia, Il Nuovo Cimento B 117, 743 (2002).

[12] R. M. Wald, General Relativity (University of Chicago Press, Chicago, 1984), Sec. 4.4.

[13] R. M. Wald, Phys. Rev. D 6, 406 (1972).

[14] S. M. Carroll, Spacetime and Geometry (Addison-Wesley, 2003), Sec. 7.2.

[15] Kip S. Thorne in Near Zero: New Frontiers of Physics, edited by J. D. Fairbank, B. S. Deaver Jr., C. W. F. Everitt, P. F. Michelson (W. H. Freeman and Company, NY, 1988).

[16] S. Gralla, A. I. Harte, R. M. Wald, Phys. Rev. D 81, 104012 (2010).

[17] B. Mashhoon, in "Reference Frames and Gravitomagnetism", in Reference Frames and Gravitomagnetism, Proceedings of the XXIII Spanish Relativity Meeting, edited by J.-F. PascualSánchez, L. Floria, A. San Miguel (World Scientific, Singapore, 2001), p. 121 [arXiv:grqc/0011014].

[18] L. Landau, E. Lifshitz, The classical theory of fields (BH-Elsevier, 1975), $4^{\text {th }}$ ed.

[19] W. Oliva, Geometric mechanics (Springer, 2002), Appendix C; Lect. Not. Math. 1798, 223 (2004).

[20] José Natário, Gen. Rel. Grav. 39, 1477 (2007).

[21] J. Costa, J. Natário, J. Math. Phys. 46, 082501 (2005).

[22] F. C. Mena, J. Natário, J. Geom. Phys. 59, 448 (2009).

[23] D. Lynden-Bell, M. Nouri-Zonoz, Rev. Mod. Physics 70, 427 (1998).

[24] M. Nouri-Zonoz, Phys. Rev. D 60, 024013 (1999).

[25] Kip S. Thorne, R. H. Price, D. A. Macdonald, Black Holes, the Membrane Paradigm (Yale Univ. Press, New Haven and London, 1986).

[26] O. Semerák, Il Nuovo Cim. B 110, 973 (1995).

[27] R. T. Jantzen, P. Carini, D. Bini, Ann. Phys. 215, 1 (1992). 
[28] R. T. Jantzen, P. Carini, D. Bini, GEM: the User Manual (2004)

http://www34.homepage.villanova.edu/robert.jantzen/gem/gem_grqc.pdf

[29] L. F. O. Costa, J. Natário, L. Wylleman, work in progress (for a brief summary of the main results, see J. Phys.: Conf. Ser. 314, 012072 (2011))

[30] J. -F. Pascual Sánchez, Il Nuovo Cimento B 115, 725 (2000).

[31] A. Matte, Canadian J. Math. 5, 1 (1953).

[32] L. Bel, Cahiers de Physique 16, 59 (1962); eng. trans. Gen. Rel. Grav. 32, 2047 (2000).

[33] D. H. Tchrakian, Gen. Rel. Grav. 6, 151 (1975).

[34] W. Campbell, T. Morgan, Am. J. Phys. 44, 356 (1976).

[35] R. Maartens, B. Bassett, Class. Quant. Grav. 15, 705 (1998).

[36] G. F. R. Ellis, R. Maartens, M. A. H. MacCallum, Relativistic Cosmology (Cambridge University Press, Cambridge, 2012).

[37] G. F. R. Ellis, General relativity and Cosmology, Proceedings of the International School of Physics Enrico Fermi, Course XLVII, edited by B. K. Sachs (1971).

[38] G. F. R. Ellis, P. A. Hogan, Gen. Rel. Grav. 29, 235 (1997).

[39] W. B. Bonnor, Class. Quant. Grav. 12, 499 (1995).

[40] C. Cherubini, D. Bini, S. Capozziello, R. Ruffini, Int. J. Mod. Phys. D 11, 827 (2002).

[41] C. B. G. McIntosh, R. Arianhod, S. T. Wade, C. Hoenselaers, Class. Quant. Grav. 11, 1555 (1994).

[42] L. Wylleman, N. Van den Bergh, Phys. Rev. D 74, 084001 (2006).

[43] L. Bel, Annales de l' I. H. P. 17, 37 (1961).

[44] V. D. Zakharov, Gravitational Waves in Einstein's Theory, Eng. Trans. by R. N. Sen (Halsted Press, John Wiley and Sons Inc, 1973).

[45] Naresh Dadhich, Gen. Rel. Grav. 32, 1009 (2000).

[46] H. Van Elst, C. Uggla, Class. Quant. Grav. 14, 2673 (1997)

[47] M. Mathisson, Acta Phys. Pol. 6, 163 (1937); Eng. Trans.: Gen. Rel. Grav. 42, 1011 (2010).

[48] W. G. Dixon, J. Math. Phys. 8, 1591 (1967).

[49] A. Papapetrou, Proc. R. Soc. London A 209, 248 (1951).

[50] F. A. E. Pirani, Acta Phys. Pol. 15, 389 (1956). 
[51] L. F. Costa, C. Herdeiro in Relativity in Fundamental Astronomy: Dynamics, Reference Frames, and Data Analysis, Proceedings of the International Astronomical Union vol 5 S261, edited by S. A. Klioner, P. K. Seidelmann and M. H. Soffel (Cambridge University Press, 2010) pp. 31-39. Preprint [arXiv:0912.2146].

[52] Charles W. Misner, Kip. S. Thorne, John A. Wheeler, Gravitation (W. H. Freeman and Company, San Francisco, 1973).

[53] I. Ciufolini, Phys. Rev. D 34, 1014 (1986).

[54] E. Massa, C. Zordan, Meccanica 10, 27 (1975).

[55] E. Massa, Gen. Rel. Grav. 5, 555 (1974).

[56] E. Massa, Gen. Rel. Grav. 5, 573 (1974).

[57] D. Bini, P. Carini, R. T. Jantzen, D. Wilkins, Phys. Rev. D 49, 2820 (1994).

[58] I. Ciufolini, E. Pavlis, Nature 431, 958 (2004); I. Ciufolini, E. Pavlis, R. Peron, New Astron. 11, 527 (2006).

[59] Review of Gravity Probe B (National Academy Press, 1995); F. Everitt et al, Phys. Rev. Lett. 106, 221101 (2011). http://einstein.stanford.edu/

[60] I. Ciufolini et al, Space Sci. Rev. 148, 71 (2009). http://www.lares-mission.com/

[61] J. Kaplan, D. Nichols, Kip Thorne, Phys. Rev. D 80, 124014 (2009).

[62] T. Damour, M. Soffel, C. Xu, Phys. Rev. D. 43, 3273 (1991).

[63] S. Deser, J. Franklin, Am. J. Phys. 75, 281 (2007).

[64] Q. G. Bailey, Phys. Rev. D 82, 065012 (2010).

[65] O. Semerák, Gen. Rel. Grav. 30, 1203 (1998).

[66] N. Drukker, B. Fiol, J. Simon, JCAP 0410, 012 (2004).

[67] K. Nordtvedt, Phys. Rev. D 7, 2347 (1973).

[68] S. W. Hawking, Astrophys. J. 145, 544 (1966).

[69] J. M. M. Senovilla, Class. Quantum Grav. 17, 2799 (2000).

[70] A. Komar, Phys. Rev. 164, 1595 (1967).

[71] F. A. E. Pirani, Phys. Rev. 105, 1089 (1957).

[72] J. Garecki, Acta. Phys. Pol. B 8, 159 (1977).

[73] P. Teyssandier, [gr-qc/9905080].

[74] C. Lozanovski and C. B. G. Mcintosh, Gen. Rel. Grav. 31, 1355 (1999). 
[75] C. Lozanovski and M. Aarons, Class. Quant. Grav. 16, 4075 (1999).

[76] S. Hawking, G. Ellis, The large scale structure of spacetime (Cambridge Univ. Press, Cambridge, 1974).

[77] P. K. S. Dunsby, B. A. C. Bassett, G. F. R. Ellis, Class. Quant. Grav. 14, 1215 (1997).

[78] M. Som, A. Raychaudhuri, Proc. Roy. Soc. A 304, 81 (1968).

[79] W. van Stockum, Proc. R. Soc. Edin. 57, 135 (1937).

[80] K. Gödel, Rev. Mod. Phys. 21, 447 (1949).

[81] I. Oszvath, E. Schucking, Class. Quant. Grav. 18, 2243 (2001).

[82] J. L. Synge, Relativity, The General Theory (North-Holland Publishing Company, Amsterdam, 1960).

[83] A. I. Nesterov, Class. Quant. Grav. 16, 465 (1999).

[84] F. K. Manasse, C. W. Misner, J. Math. Phys. 4, 735 (1963).

[85] V. Bolós, Commun. Math. Phys. 273, 217 (2007).

[86] V. Bolós, J. Geom. Phys. 56, 813 (2006).

[87] L. Wylleman, D. Beke, Phys. Rev. D 81, 104038 (2010).

[88] F. Dahia, P. F. Silva, [arXiv:1004.3937].

[89] W. Campbell, J. Macek, T. Morgan, Phys. Rev. D 15, 2156 (1977).

[90] I. Ciufolini, Nature 449, 41 (2007)

[91] A. García-Parrado Gómez-Lobo, Class. Quant. Grav. 25, 015006 (2008).

[92] J. J. Ferrando, J. A. Sáez, Class. Quant. Grav. 29, 075012 (2012).

[93] G. Rizzi, M. L. Ruggiero, Relativity in Rotating Frames, edited by G. Rizzi, M. L. Ruggiero (Kluwer Academic Publishers, Dordrecht, 2004), preprint [gr-qc/0305084]; G. Rizzi, M. L. Ruggiero, Gen. Rel. Grav. 35, 1743 (2003); M. L. Ruggiero, Gen. Rel. Grav. 37, 1845 (2005).

[94] L. Bel, Colloques Internationaux du Centre national de la reserche scientifique, p. 119, Paris (1962).

[95] M. Soffel, S. Klioner, J. Muller, L. Biskupek, Phys. Rev. D 78, 024033 (2008).

[96] T. W. Murphy Jr., K. Nordtvedt, S. G. Turyshev, Phys. Rev. Lett. 98, 071102 (2007).

[97] K. Nordtvedt, Int. J. Theoretical Physics 27, 2347 (1988).

[98] D. Bini, A. Geralico, R. Jantzen, Class. Quant. Grav. 22, 4729 (2005). 
[99] D. Mason, C. Pooe, J. Math. Phys. 28, 2705 (1987).

[100] W. G. Dixon, Proc. Roy. Soc. Lond. A 314, 499 (1970).

[101] A. Balakin, J. Van Holten, R. Kerner, Class. Quant. Grav. 17, 5009 (2000).

[102] J. M. Bardeen, Astrophys. J. 162, 71 (1970).

[103] J. M. Bardeen, W. H. Press, S. A. Teukolsky, Astrophys. J. 178, 347 (1972).

[104] O. Semerák, Gen. Rel. Grav. 25, 1041 (1993).

[105] O. Semerák, Class. Quant. Grav. 13, 2987 (1996).

[106] L. Iorio, H. Lichtenegger, M. L. Ruggiero, C. Corda, Astrophys. Space Sci 331, 351 (2010).

[107] Eric Gourgoulhon, 3+1 Formalism and Bases of Numerical Relativity, [gr-qc/0703035].

[108] R. Arnowitt, S. Deser, C. W. Misner, in Gravitation: an introduction to current research, edited by L. Witten (Wiley, New York, 1962), pp.227-264; preprint [gr-qc/0405109].

[109] T.W. Baumgarte, S. L. Shapiro, Phys. Rep. 376, 41 (2003).

[110] C. Tsagas, Class. Quant. Grav. 22, 393 (2005).

[111] C. Tsagas, Phys. Rev. D 81, 043501 (2010); J. Barrow, C. Tsagas, K. Yamamoto, Phys. Rev. D 86, 023533 (2012).

[112] D. Bini, F. de Felice, A. Geralico, Class. Quant. Grav. 23, 7603 (2006).

[113] David C. Kay, Tensor Calculus (McGraw-Hill, 1988).

[114] H. Goldstein, C. Poole, J. Safko, Classical Mechanics (Addison-Wesley, 2000), 3rd ed. 